\title{
Putting Maps Online:
}

Exploring A Web-Based Geospatial Information System

By A Case Study of the Online Ottawa Housing Atlas

\section{By}

\author{
Li Yu \\ A thesis submitted to \\ the Faculty of Graduate Studies and Research \\ in partial fulfillment of \\ the requirements for the degree of
}

\section{Master of Arts}

Department of Geography and Environmental Studies

Carleton University

Ottawa, Ontario

September, 2002

(C) 2002, Li Yu 
National Library

of Canada

Acquisitions and Bibliographic Services

395 Wellington Street Ottawa ON K1A ON4 Canada
Bibliothèque nationale

du Canada

\section{Acquisitions et}

services bibliographiques

395, rue Wellington

Ottawa ON K1A ON4

Canada
The author has granted a nonexclusive licence allowing the National Library of Canada to reproduce, loan, distribute or sell copies of this thesis in microform, paper or electronic formats.

The author retains ownership of the copyright in this thesis. Neither the thesis nor substantial extracts from it may be printed or otherwise reproduced without the author's permission.
L'auteur a accordé une licence non exclusive permettant à la Bibliothèque nationale du Canada de reproduire, prêter, distribuer ou vendre des copies de cette thèse sous la forme de microfiche/film, de reproduction sur papier ou sur format électronique.

L'auteur conserve la propriété du droit d'auteur qui protège cette thèse. Ni la thèse ni des extraits substantiels de celle-ci ne doivent être imprimés ou autrement reproduits sans son autorisation.

\section{Canadä'}




\section{ABSTRACTS}

This study provides a vision of the opportunities and challenges offered by the Internet technologies to the mapping and GIS industry through an exploration of the different strategies in putting geo-spatial data online. A simulation process of distributing geospatial data via the Web has been established through a case study of the Online Ottawa Housing Atlas (OOHA).

The OOHA has been developed based on an open source Web mapping system, MapServer, and resides on the Carleton University Website. This case study examines the proposed fundamental approaches to the Web mapping application and demonstrates the basic interactive spatial manipulations via the Internet. Evidence demonstrates that considerable socio-economic value has been created through the OOHA. The OOHA provides information on housing conditions and housing needs within the Ottawa region to the public and government organizations by portraying the patterns of geographic variation of housing related demographic, economic and market factors in Ottawa. This research also aims to advance the roles that web mapping and WebGIS technology play in society through the case study of OOHA.

In conclusion, this study has presented a discussion of the advantages and limitations of integrating the Internet with GIS technologies, the approaches of implementing an online geospatial information application and the potential of Web mapping/WebGIS technology in society. 


\section{ACKNOWLEDGEMENTS}

This research would not be possible without the help and support of many people.

I would like to thank my supervisor Prof. Fraser Taylor, Department of Geography, Carleton University, and my adviser Prof. Christine Earl, Department of Geography, Carleton University, for their guidance and support during the past two years. Dr. Taylor and Prof. Earl have always encouraged me to strive for the highest possible standards in my studies and encouraged my enthusiasm about my topic and study.

I also wish to extend a thank you to all the faculty, staff and classmates of the Geography Department, Carleton University as well as my friends, who contributed in many forms and supported my research. It is your kind help that has made my student life more enjoyable. A special thank you to Ms. Hazel Anderson, who has always been helpful and supportive, and has made me feel warm and pleasant throughout the two-year study.

Finally, my deepest thank you to my parents, who have always supported and encouraged me in my life.

FOR MY PARENTS, MRS. JUNLING ZHAO and MR. SHAOCHEN YU! 


\section{TABLE OF CONTENTS}

ACCEPTANCE SHEET.

.ii

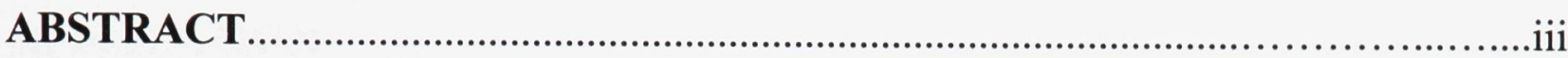

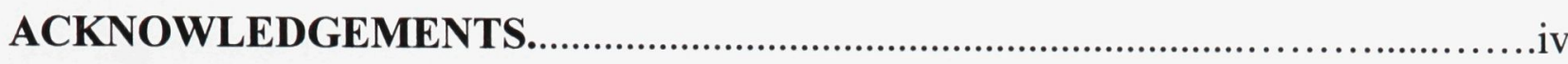

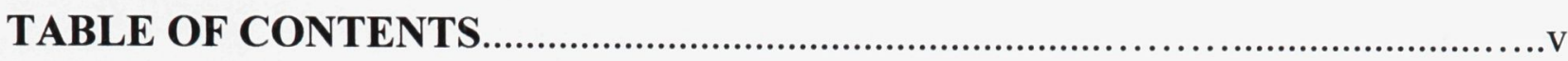

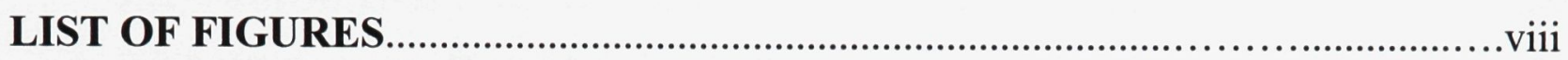

\section{CHAPTER 1. INTRODUCTION}

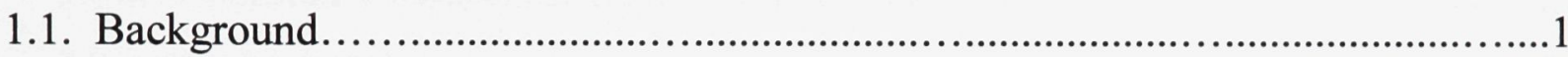

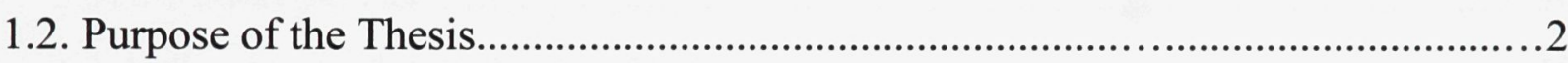

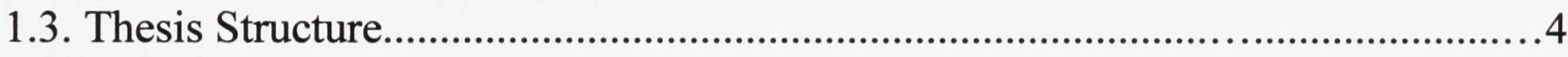

CHAPTER 2. WEB MAPPING TECHNOLOGY AND PUTTING MAPS ONLINE

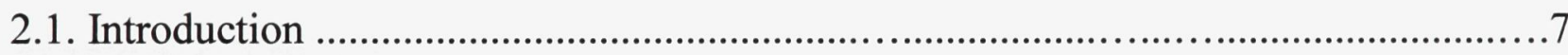

2.2. Internet Technology ….......................................................................................

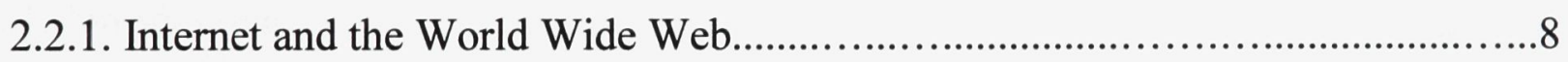

2.2.2. Client/Server Architecture........................................................................ 9

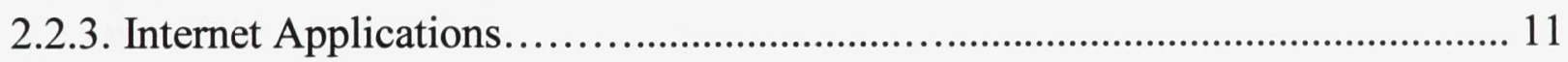

2.3. Web Mapping Technology ..................................................................................... 12

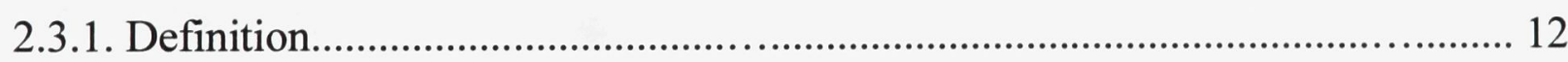

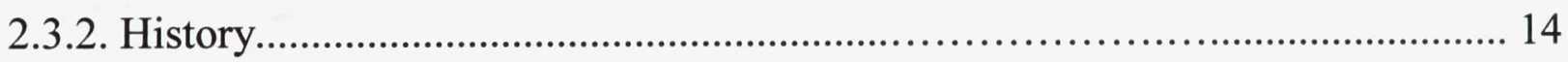

2.3.3. Advantages and Limitations...................................................................... 15

2.4. Strategies of Web Mapping Applications............................................ 18

2.4.1. Web Mapping Service Architecture.................................................................18

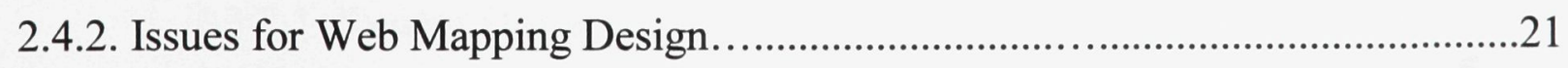

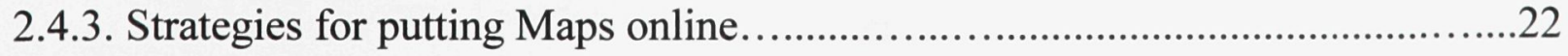




\section{CHAPTER 3. CURRENT WEBGIS SYSTEMS AND WEB MAPPING}

\section{APPLICATION}

3.1. Introduction .29

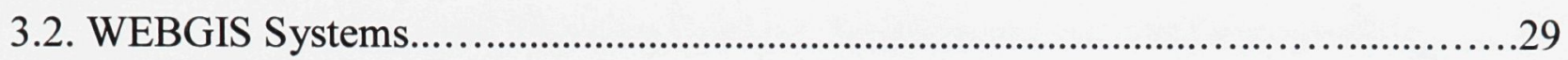

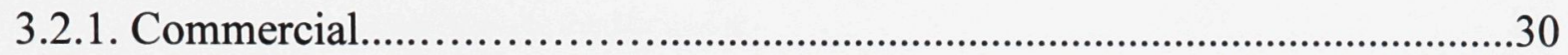

3.2.2. Opensource Web Mapping Software .....................................................43

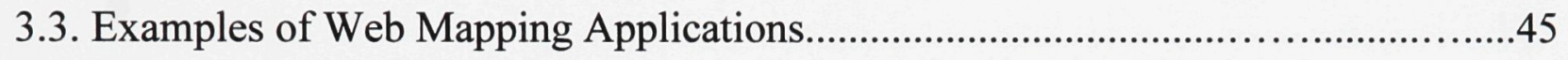

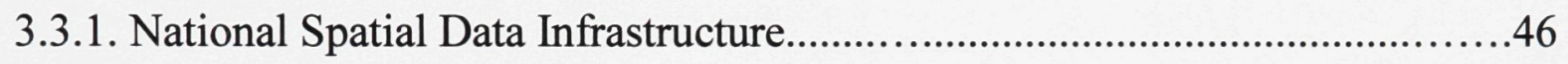

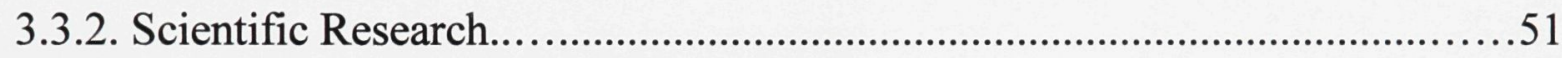

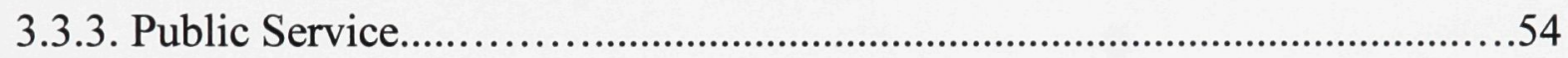

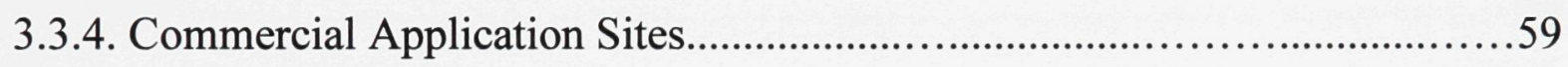

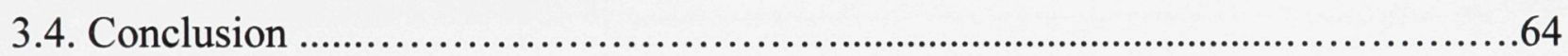

\section{CHAPTER 4. A CASE STUDY - CONSTRUCTING ONLINE OTTAWA HOUSING ATLAS}

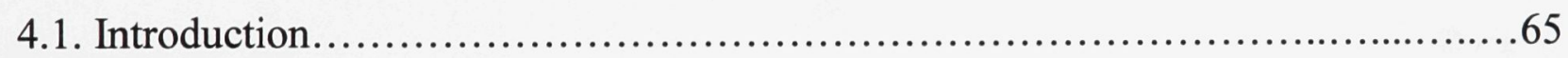

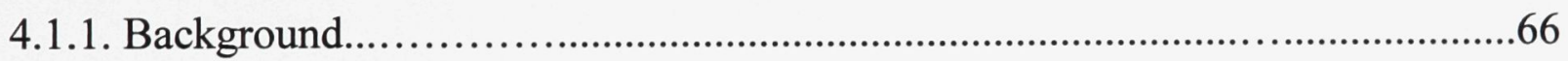

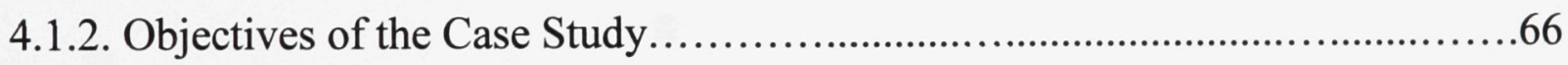

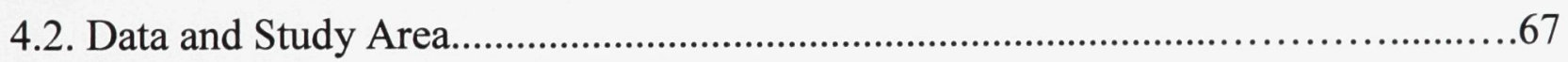

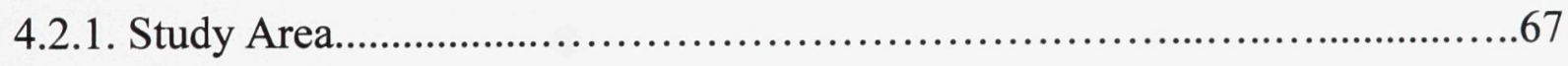

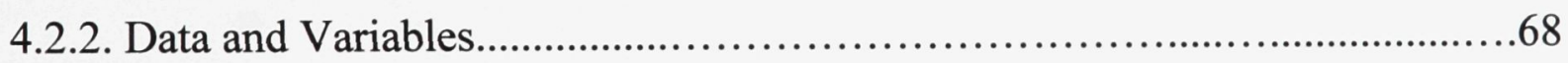

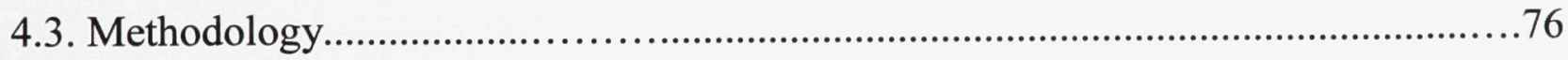

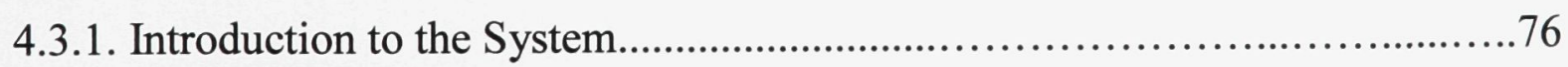

4.3.2. System Design and System Development...............................................77

4.3.2.1. System Design......................................................... 77

4.3.2.2. System Development............................................. 78 


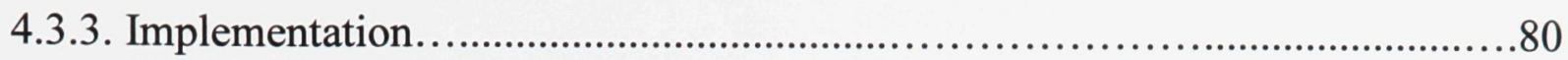

4.3.3.1 Issues for OOHA Site Design................................................. 80

4.3.3.2 Content Interface and Functionality..........................................84

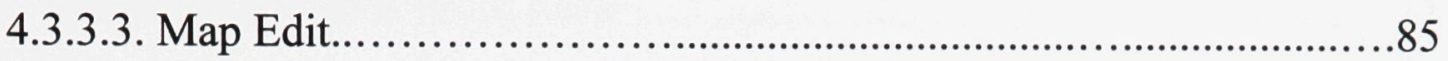

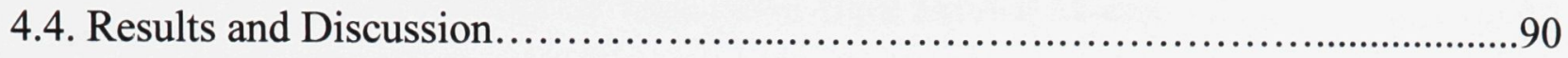

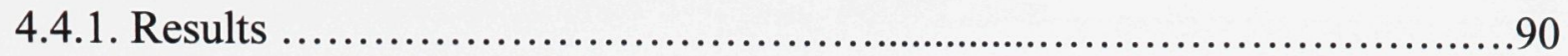

4.4.1.1. Regional Housing Context: SocioEconomic and Demographic.......90

4.4.1.2. Housing Needs Indicators...............................................97

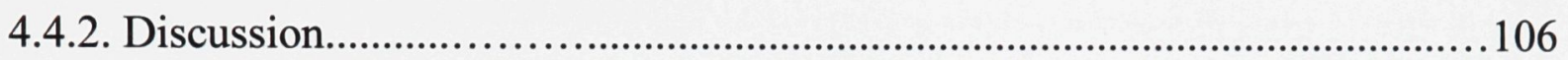

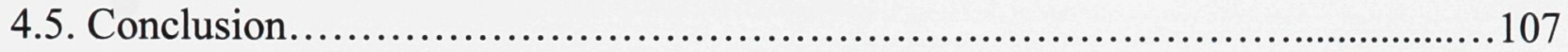

\section{CHAPTER 5. CONCLUSION AND OUTLOOK}

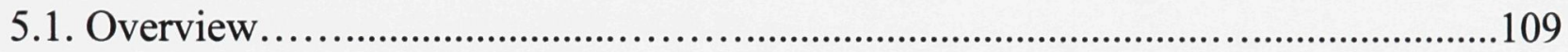

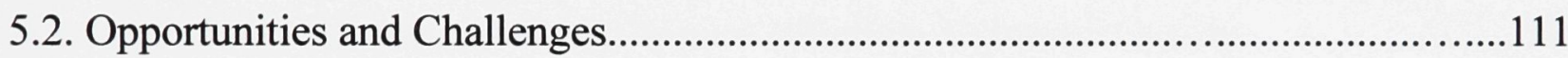

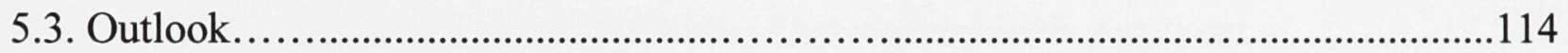

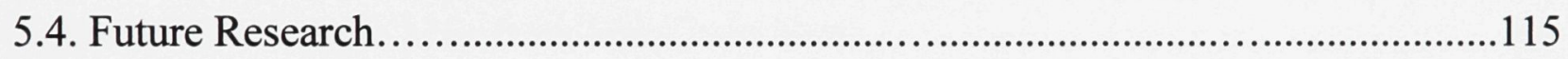

APPENDIX: Glossary of Internet Terminology ...............................................116

REFERENCES 


\section{LIST OF FIGURES}

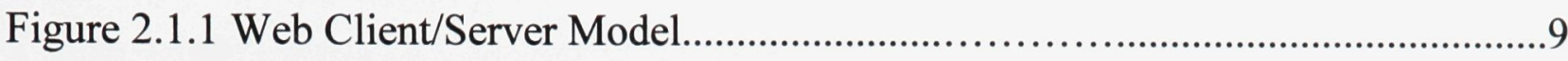

Figure 2.1.2 Internet Application Work Flow.......................................................11

Figure 2.4.1 A Basic Client/Server Geospatial Data Service Model...........................18

Figure 2.4.2 Workflow Chart of A Web Mapping Application..................................22

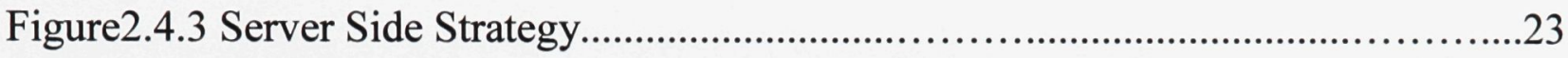

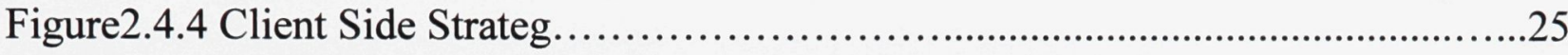

Figure 2.4.5 Hybrid Client/Server Strategy ...........................................27

Figure 3.3.1 National Atlas of the United States........................................................49

Figure 3.3.2 Basic Interface of the National Atlas of Canada......................................51

Figure 3.3.3 Interface of the Atlas of Antarctic Research..............................................54

Figure 3.3.4 City of Ottawa- the Atlas of Ottawa Website Interface...........................56

Figure 3.3.5 Cabarrus County GIS Web Page …....................................5 57

Figure 3.3.6 Land Information Application Interface, Cabarrus County....................58

Figure 3.3.7 Land Parcel Table of Cabarrus Land Information Application..................59

Figure 3.3.8A Sample Map from MapQuest Site.................................................60

Figure 3.3.9 Housing Property Query: Select Ottawa Area.......................................62

Figure 3.3.10 Housing Property Query: Select A Property Type.....................................62

Figure 3.3.11 Housing Property Query: Fill in Search Criteria....................................63

Figure 3.3.12 Housing Property Query: Result Display........................................63

Figure 4.3.1 Online Ottawa Housing Atlas Application Server Side Strategy

Model..........................................................77

Figure 4.3.2 Online Ottawa Housing Application Interface......................................... 82

Figure 4.4.1 Housing Economic Factors 1991 .....................................................92

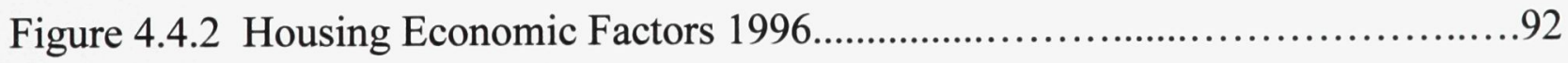

Figure 4.4.3 Housing Economic Feature Query 1991/1996.................................93

Figure 4.4.4 Housing Economic Factors 1991/1996 Information...........................94 


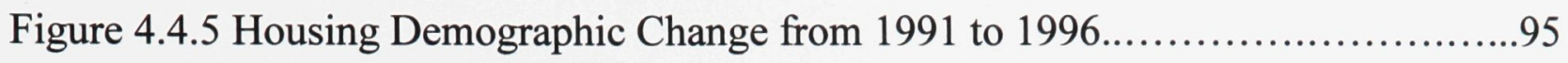

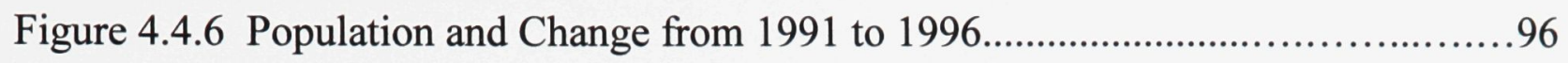

Figure 4.4.7 Household Characteristics Map1991................................................101

Figure 4.4.8 Household Characteristics Map1996...................................................101

Figure 4.4.9 the Number of Household Change Distribution 1991-1996 ....................102

Figure 4.4.10 Multiple Query of Household Characteristics Map............................103

Figure 4.4.11 Single Query of Household Characteristics Change..........................104

Figure 4.4.12 Kanata City Household Characteristics Change................................105 


\section{CHAPTER 1. INTRODUCTION}

\subsection{Background}

Today, with the rapid development of computer network technology, people can manage and disseminate vast quantities of information with speed and flexibility. Computing power, computer hardware, GIS software and the development of large databases continue to increase significantly and this had led to fundamental changes in the geotechnology industry.

The growing popularity of the Internet has made it an integral part of many societies. The efficient data dissemination and dynamically interactive communication features of the Internet make it of real value to the emerging GIS architectures, named as the Geospatial Network Architecture, for spatial information service via Internet protocols (Plewe, 1997). This promises to leverage the works of cartographers and also GIS professionals. It radically enlarges the use of geo-spatial information and GIS functionalities in society. What is emerging now is something called "societal GIS"1 (Harder, 1998), an electronic web of geo-spatial information systems that everyone can access.

It is noticeable that the convergence of Geographic Information Systems (GIS) and World Wide Web technology are jointly influencing the traditional mapping industry, and greatly changing conventional ways of both map production and map use. This

\footnotetext{
${ }^{1}$ Note: "Societal GIS" is a state in which geographic information is part of the framework of our conscious lives, and touches every aspect of daily existence (Harder, 1998).
} 
convergence adds valuable means and exciting perspectives to the map industry. Distributing geo-spatial information to the public through the Internet becomes possible in the context of today's technology and some of these online mapping applications are already in use in recent years, such as MapQuest (http://www.mapquest.com), MapBlast (http://www.mapblast.com). Users can conduct dynamic spatial analysis, interactively manipulate geographic data via the Web to generate map products specifically to meet their own needs. The powerful data dissemination and dynamically interactive communication features of the World Wide Web make geo-spatial data more easily and efficiently shared with multiple users.

Putting maps online is important to satisfy new mass demands in the information era. Web-based spatial analysis geographic network architecture must be constructed by integrating geo-spatial tools with the Internet. New challenges and opportunities are created for the map industry by this remarkable change and the latest innovative technology available for web mapping.

\subsection{Purpose of the Thesis}

The purpose of this thesis is to provide a vision of the new opportunities and challenges offered by these recent and rapidly growing Internet technologies for mapping and the GIS industry through exploring different approaches of putting geo-spatial information online. A simulation of the process of distributing geographic information, including spatial datasets, socio-economic statistics data, text files, images, via Internet will be established. It will demonstrate the basic dynamic and interactive geospatial 
manipulations based on the capability of a Common Gateway Interface(CGI) MapServer engine. Furthermore, an understanding to the advantages and limitations of integrating Web technique with GIS functionalities, and the future trend of this particular realm will also be discussed.

In this research, Ottawa housing information was used as a case study to illustrate one approach to put geospatial data online. A web-based Ottawa Housing Atlas framework has been developed on the existing Carleton HTTP server so that the online Ottawa Housing Atlas can be published via Carleton University's Website.

A case study is used to examine the proposed fundamental approach to the establishment of the Web based geo-spatial network architecture through the development of an online Ottawa Housing Atlas. Moreover, there is also a considerable socio-economic value in exploring the creation of a Web based Ottawa Housing Atlas. Housing data is one of the most important indicators of quality of life. It provides valuable information to both the public and government organizations by portraying socio-economic spatial variation. Many approaches have been employed in the past to utilize housing statistics to monitor housing requirements as well as the related economic issues in society. However, this case study will attempt to explore the process of distributing spatio-statistical data on housing through the Internet so as to provide the most up-to-date and reliable information to governments, private agencies and the public. The online housing atlas will demonstrate the housing condition and housing needs in the Ottawa region by studying the pattern of spatial and temporal variations of housing related demographic, economic 
and market factors associated with housing issues in Ottawa.

This study hopes to advance the roles web mapping plays in the application of geographic information by conducting a case study of the Online Ottawa Housing Atlas (OOHA) and a series of comprehensive investigations of current WEBGIS applications. It is possible that, by moving maps online, more and more spatial information can be distributed in the regional community which can create considerable benefits to the public by sharing and utilizing these information.

\subsection{Thesis Structure}

The thesis consists of five chapters organized as following:

\section{CHAPTER 1: INTRODUCTION}

This chapter introduced the context of the thesis research including: the current status of web mapping technology and the origin of the topic - why it is important to conduct this research? The purpose of this research and the thesis organization are given in this section.

\section{CHAPTER 2: WEB MAPPING TECHNOLOGY AND PUTTING MAPS ONLINE}

This section provides the concepts of Web Mapping technology and the different strategies of how to put maps online. It introduces what web-map and WebGIS are, the features that distinguish them from the other Internet applications, and why Internet technology is an important medium for presenting and disseminating geospatial information. It will describe and compare different approaches of putting maps online, 
including their strengths and weaknesses.

\section{CHAPTER 3: WEBGIS SYSTEMS AND APPLICATIONS}

In this chapter, an investigation of the existing web-based geospatial information distribution systems (varieties of WebGIS, Map Server) and applications is conducted. This will include the existing commercial software, freeware and web mapping application examples such as: MapQuest, National Atlas of Canada (http://www.atlas.gc.ca). MapQuest is a popularly used professional online road atlas site which was developed by Geosystem Global Company. The National Atlas of Canada is a government Web atlas site that provides national scale geographic information on physical, human, economic, historic geographic theme and some relevant Canadian issues such as health, climate change. This detailed information will be introduced in Chapter 3.

CHAPTER 4: A CASE STUDY - CONSTRUCTING AN ONLINE OTTAWA HOUSING ATLAS

In this part, a simulated web based geospatial information application, 'Online Ottawa Housing Atlas', will be built-up to illustrate the fundamental strategy and process of Web based spatial information network architecture. Then, by clicking 'go', the Online Ottawa Housing Atlas application will be launched through the Carleton University Web server. The URL of this application is

“ http://www.carleton.ca/ lyu/Happy Housing.HTM". 
This study will endeavor to explore the valuable roles that web mapping is playing in society by presenting spatio-temporal features of Ottawa housing data via the Internet. Therefore, the approach of serving society by interactive web mapping is examined and demonstrated through establishing this Online Ottawa Housing Atlas case study via Carleton University's Website.

\section{CHAPTER 5: CONCLUSION AND OUTLOOK}

This chapter will summarize and conclude the research. It will address the opportunities and challenges, the outlook and future prospects of web mapping technology and applications.

An appendix, 'Glossary of Internet Terminology', is included. 


\section{CHAPTER 2. WEB MAPPING TECHNOLOGY AND PUTTING MAPS ONLINE}

\subsection{Introduction}

As a cutting-edge technology, Web mapping is a convergence of multi-disciplinary knowledge in a variety of areas including GIS, Cartography, the Internet, computer hardware and software. To create functional interactive maps on the World Wide Web requires consideration of many different aspects of each discipline. The structure of the Internet is one of the fundamental elements for making maps work online. However, it will be difficult to talk about this technology in even general terms without some background on how the Internet, specifically how the World Wide Web, actually works. For the purpose of this research, a thorough understanding of the structure of Internet is important for deciding a proper site configuration (site strategy design), making interactive maps deliverable via the Internet, so as to successfully construct a map-based Web application. Hence, it is fitting to start by discussing the basic conceptual background of Internet knowledge and Web mapping by defining the key terms used in this research. Three primary site configuration strategies, to create online maps, will also be described in this section.

\subsection{Internet Technology}

Internet technology has come a long way, but it emerged as a dominant force in global communications only from the middle of the 1990s. Today, it has obviously become part of the infrastructure of society around the world. 


\section{Internet and the World Wide Web}

The Internet is a global network of networks through which computers communicate. It consists of millions of computers, various types of hardware and software, physically connected to one another and communicating globally via different types of protocols, sets of conventions that govern how packets of data are sent across the computer network. No single organization owns or controls the entire network, although it is generally controlled by a series of accepted standards. The Internet is often referred to as a "kludge that works" (Plewe, 1997)

The World Wide Web (WWW or Web, hereafter) is one form of communication that can take place over the Internet, alongside other forms such as electronic mail, Telnet, FTP, CHAT, etc. Although based on the foundation of the HTML (Hypertext Markup Language) data format and HTTP communication protocol (Hypertext Transfer Protocol), it has grown to include many types of information such as graphics, scripts and applets. One of the outstanding features of the Web is that it is virtually platform independent which enables the data to be served by different types of computers running various operation systems such as Windows, Unix, Linux and AppleOS.

Conceptually, the World Wide Web is mainly based upon the concept of hypertext, where most of the information is portrayed as documents. Certain parts of each document (including text and graphics) have embedded pointers to other documents. Therefore, users can browse the information by following these links. (Brandon Plewe, 1997) The 
architecture of the World Wide Web is a client/server model for distributing and accessing multimedia documents on the Internet.

\section{Client/Server Architecture}

Client/server architecture on which the World Wide Web and most other Internet services are based is illustrated in figure 2.1.1 below:

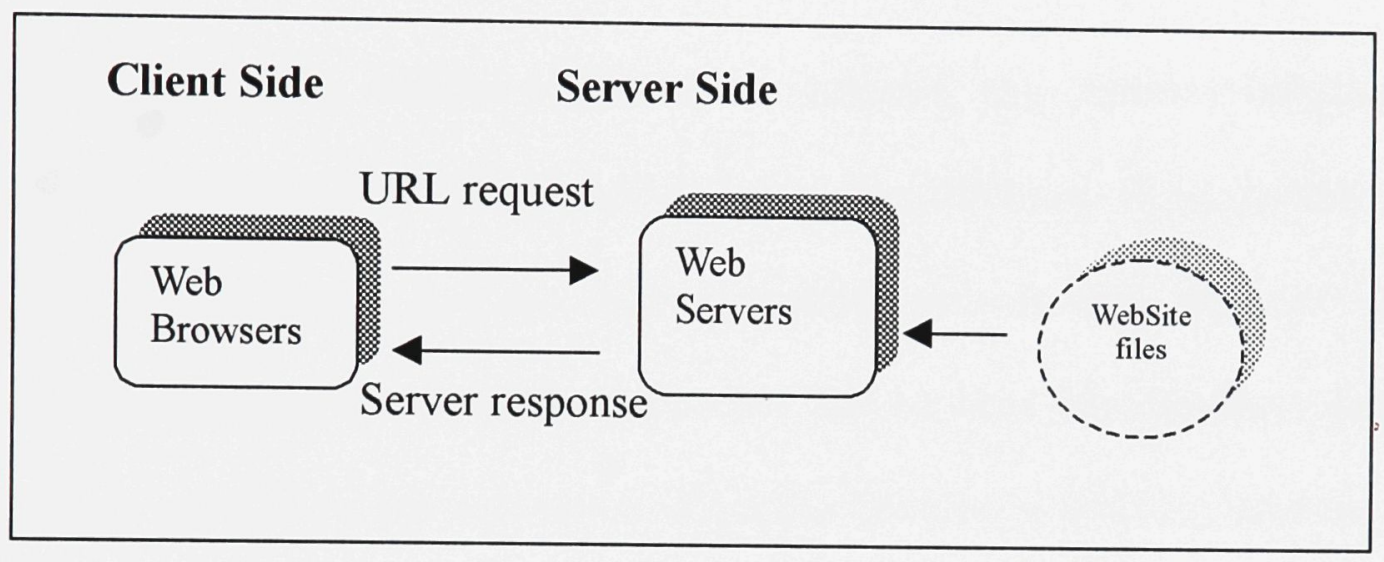

Figure 2.1.1 Web Client/Server Model

The client/server architecture has three fundamental components: Web (client) browsers, network (Internet) and Web servers. It consists of Web browsers and Web servers that communicate across the computer network following an established communication protocol. The computer network refers to either the Internet or a secure closed private network called intranet. An Intranet also operates on Internet standards, i.e. protocols. The unifying protocol is the Internet Protocol (IP), which allows heterogeneous hardware and software environments to communicate effectively. 
A Web browser is the most common client program that enables users to retrieve and view information. When a user types a specific Web address, called a URL (Uniform Resource Locator which locates the resources on the Web), into the Web browser, the browser program will send a request to the server computer located at that URL address. The server will then load a file from its disk and transmit it over the Internet to the client Web browser.

On the server side, besides HTTP server software, (e.g. Internet Information Services (IIS) on Windows NT/2000, Personal Web Server(PWS) on Windows98/95), there are a blend of technologies that are working together such as databases and middleware, to provide increasingly sophisticated functions and services. Middleware refers to the script software residing on the web server. It has the function of dividing information into small packages and checking their receipt by the client computer. The middleware tier includes the technologies like Common Gateway Interface (CGI) which is a protocol for adding specialized processing capabilities to the HTTP server. A CGI script, which is usually written in Perl or $\mathrm{C}$ languages, is an independent program that processes data based on certain parameters. When the browser makes a request to the server, the server recognizes the request as the domain of a particular CGI program. The parameters are passed to the CGI for processing. The results will be then transmitted back to server, and the server will send the information back to the client browser. For example, some WebGIS software, such as Intergraph's GeoMedia WebMap and Minnesota University's MapServer, is implemented in CGI. However, ESRI's MapObjects is executed in Active Server Pages (ASP which is similar in function to CGI script and usually written in VB 
script or Java script. MapObjects runs on Microsoft IIS or PWS).

\section{Internet Applications}

Internet applications (Web applications) are referred to as the online applications that users can access via a Web browser. This provides the dynamic and interactive response that is distinct from the regular web sites that only provide static content. The Internet applications operate in the background when a request is rendered to the server such as, filling out an online registration form, making an online purchase, requesting a stock chart for a given time range or finding a new house location meeting your requirements. The dynamic content is created by the relevant Web applications using the enabling technologies such as CGI, PHP, ASP or Java servlets. Figure 2.1.2 gives the procedures for how an Internet application works.

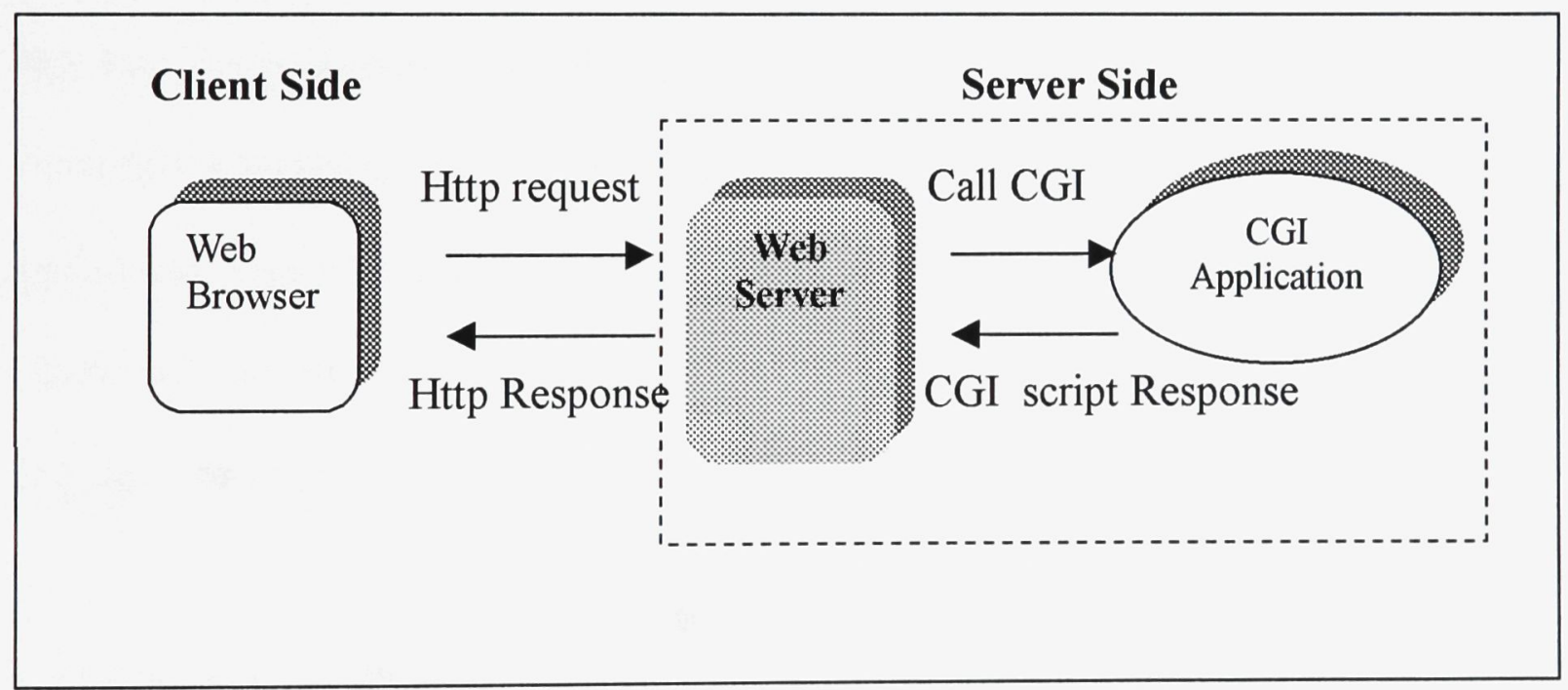

Figure 2.1.2 Internet Application Work Flow

The figure illustrates the following steps, 
- when the web server gets a CGI request, it executes the application in a separate process,

- the server passes the parameters received with the request (if any) to the application and collects the output,

- this output is then returned to the client just as if it had been retrieved from a static document.

Currently, the Internet, originally a text-based communication tool, has developed into a multimedia communication network. A global hypermedia infrastructure has been realized in the form of the World Wide Web. In addition, two dominant Web browsers: Microsoft Internet Explorer and Netscape Navigator provide easy to use graphic user interfaces. They both support the standard HTTP communication protocol, and the most popularly used HTML, Dynamic HTML data format internally. This allows a computer to link with the information outside the original content domain, thus increasing the Internet potential as a spatial data distribution and presentation tool for the public. Furthermore, additional plugins added onto the plain HTML of client side and CGI applications developed on server side have largely broadened the possibilities of Internet for implementing spatial data in more intelligent and interactive way.

\subsection{Web Mapping Technology}

\subsubsection{Definitions}

As the Web holds promise for exponential increases in the efficiency and effectiveness of data dissemination, a broad array of mapping products and services have been integrated with the Internet technologies to enable people to access geographic information online in 
a variety of forms including maps, graphics, images, text and data. Terms such as the Web (Internet) mapping, WebGIS, map servers are used by the Internet and GIS community at present. There is sometimes confusion about the use of these terms. Thus, it is necessary to define the meaning of different Web-based geospatial application terms for the purpose of this paper.

As the name indicates, Web mapping (also called the Internet mapping) is a term used to describe the creation of maps and geographic data dynamically on the Web so as to allow geographic communication with specific users or with users all over the world via the Intranet/Internet. New characteristics, such as dynamic and interactive map presentation, multimedia integration, have been added onto the map by Internet technologies. With respect to the emphasis on conducting sophisticated GIS functions dynamically through the Internet, WebGIS (Internet GIS) is usually used instead of the more generic Web mapping (or Internet Mapping) term.

"Putting maps online" in this study is meant to be inclusive. It covers the generic applications and services that use Internet to make geospatial information available to a wider audience. This study is focused on an implementable approach of putting geospatial information online. Therefore, both Web mapping and WebGIS (Internet GIS) fall within the scope of this study. Both terms are used in the broad sense of geospatial data and service distribution and presentation through World Wide Web.

Many varieties of web-based geospatial applications and products, such as Web (Internet) 
mapping, WebGIS, Internet Map Server application, are available. Some representative examples will be introduced and discussed in Chapter 3 .

\subsubsection{History}

The Internet was created about 30 years ago. It has been around almost as long as GIS technology. However, only in recent years has the Internet emerged as part of mainstream society. In addition, the Internet is recognized as one of the most powerful forces shaping GIS since middle of the 1990 s. Although it is perhaps too new to be addressed as "history", nobody will deny that the Internet has greatly influenced the GIS industry.

Initially, the Web utilized mainly text and images. It was largely below most people's horizon of awareness. Things started to change in the early 1990s. The first Web based interactive Map Server was created in 1994. Xerox PARC Map Viewer put up the first Web page with an active map as an experiment in interactive information retrieval (Harder, 1998). As the first Internet mapping site, the developers had no easy way to provide needed functionalities. There were no forms and no Internet-specific programming languages like Java at that time. The page was pure HTML, with a series of hyperlinks for selecting options. The Xerox PARC Map Viewer was discontinued in 1997 after four years service. However, it helped spawn other ideas for incorporating maps into the Web and has verified that spatial information is an extremely useful part of many Web applications.

The introduction of new Web technologies, such as Java and Active X, made it possible 
to develop Internet GIS for a variety of needs. Geospatial data producers who often spend millions of dollars in printing and distributing paper maps have recognized the Internet as a cost-efficient medium to publish large amounts of data online. It should also be noted that phone companies' Internet yellow pages were actually among the earliest Internet mapping applications.

Today, Web mapping applications are being provided by almost all the geomatics organizations. The developments in web mapping are also changing as fast as internet/Web technologies, as one depends on the other. What was up-to-date last year will be almost certainly rapidly become out of date as the life of a specific web technique knowledge is often very short. Generally speaking, Internet geospatial applications can be built with a variety of object-oriented languages such as Java, PHP, Visual Basic, Visual $\mathrm{C}++$ or DELPHI. They can support active $\mathrm{X}$ Controls. On the client side, people prefer to access geographic data directly by means of the programs of the standard web browser, Internet Explore or Netscape Navigator, rather than use a specific GIS browser such as ArcExplorer.

\subsubsection{Advantages and Limitations}

The newly emergent and fast changing Internet technologies have brought the mapping industry both advantages and new challenges

\section{Advantages}

As a combination of mapping and Internet technology, Web mapping holds the potential 
to efficiently distribute geospatial information to a very large world wide audience. Thus, it opens new possibilities of geospatial information applications such as geographical communication with other people in presenting ideas and integrating information, or optimal locational decision making through the Internet. With the increase of digital data resources and the expansion of the Web, data sharing becomes more important and convenient. Also, the interactive feature of the web based map often allows users to query data, to derive more useful information and to integrate social, economic aspects of data with geographic information in dynamic fashion. This has advantages over traditional maps that are static. Seeking information dynamically on the location of the nearest and cheapest hotel is a simple but powerful example.

The near term future will see the fundamental functionalities and content of GIS online and universal access and use through the Web. Internet sessions will become more interactive. The number of web site will increase and more of them will include the ability to process and manipulate data. GIS users will be able to implement GIS applications without purchasing and owning large amounts of GIS related geo-processing software. An important innovation of "pay for use", a consumer-directed Web mapping and GIS services (Waters, 2001) will emerge.

Moreover, there is a growing trend in using web mapping for government services. Institutions such as federal agencies collect, use, and supply monitoring information in real time and consequently, dynamic data sharing plays an important role that will be surely improved through WebGIS technology. Maps can be generated quickly from huge 
databases of spatial information and distributed immediately to organizations as needed all over the world via the cost-effective and efficient Web medium. It is also possible to add GIS functionality to a wide range of network-based applications in business, government, and education organizations to strengthen data sharing.

In addition, Internet mapping opens a new source of accessing geographical data very quickly. Accessing and searching paper maps to get location data can be very timeconsuming whereas over the web, this can be done in a matter of minutes.

\section{Limitations}

There are also limitations with this very promising geospatial data distribution medium. The most outstanding issue is the Internet bandwidth limitation. Data transfer is affected by the E-speed of the Internet connection and transmission. As the Internet grows (more computers trying to send data simultaneously), and becomes more complex (i.e. multimedia objects such as video, image constitute much larger files), the need for bandwidth is constantly increasing. Whether the current Internet technology can be upgraded fast enough to keep up with demand is a question.

Another important consideration is people's perception of maps. There is geographic illiteracy existing in the general public. General consumers of Internet resources may not understand geographic principles and map information. This lack of understanding creates barriers for geographic data use and distribution. It has been argued that one of the main reasons MapQuest does well is that people do understand conventional road 
maps. However, maps for special purposes that are unfamiliar to people such as decision support systems are harder for the general public to understand. Most people do not know the cartographic principles geographers and cartographers take for granted, such as scale bars, north arrows and thematic shading patterns. Many maps created by E-commerce companies are very low quality maps. Such maps on the Internet often appear without, a scale bar, legend, or use color inappropriately to communicate information. These probably will greatly affect the use of Web mapping applications.

\subsection{Strategies of Web Mapping Application}

\subsubsection{Web Mapping Service Architecture}

There are many ways in which geographic information can be distributed on the Internet, but they are basically founded on the same general design. The basic architecture is similar to the client/server model, a model upon which the Web and most other Internet services are based. A general client/server model of geospatial data service framework is shown in Figure 2.4.1

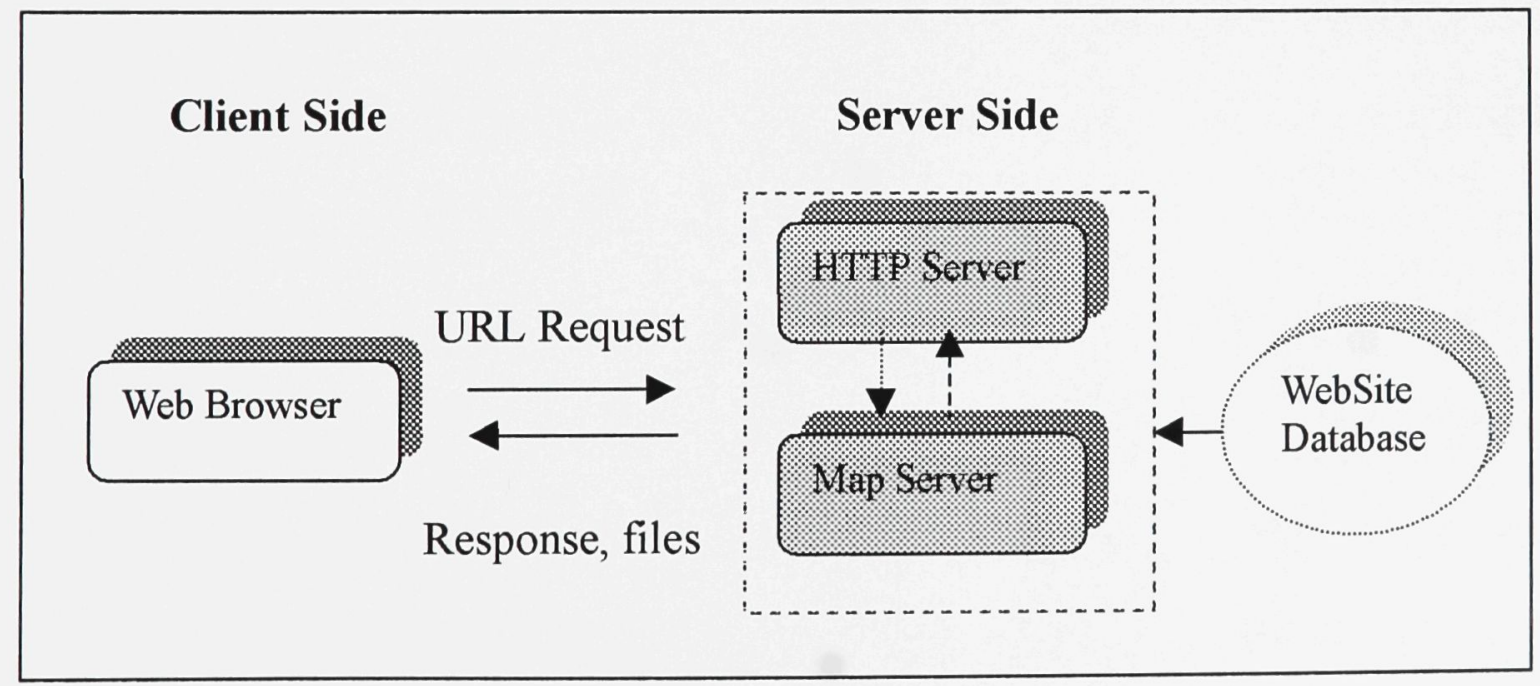

Figure 2.4.1 A Basic Client/Server Geospatial Data Service Model 
The above model is an extension of the client/server concept. The server part is a multitiered server. A client is typically a Web browser or it is even possible to conceive of an entire GIS program as a geospatial data service client. The multi-tiered server consists of a normal web server and a running map server program - usually a CGI script that consists of basic spatial data processing functions. When someone makes a request for a map or other GIS function from the client side, the request message is sent over the Internet to the HTTP Server. The HTTP server recognizes if it is a spatial data/processing request, then passes it to the map server program which translates it into some type of internal code (e.g. queries or map drawing commands) and processes the request.

It should be noted that most of the current map server CGI programs are still not capable of very complicated GIS functionalities. In the case of sophisticated GIS operation request, the multi-tiered server necessarily consists of a GIS program in addition to the Web server and the map server, which is responsible for providing the sophisticated GIS functionality and which return the results back to the server. These can be in the form of a map, text, image, or even raw data files. The map server will then reformat the output into an Internet standard format, or at least something understood by a browser plug-in or Java applet (an applet is a module that extends the functionality of a Web Browser). The reformatted information is returned via the Web server back to the client where it will be presented as a Web page. This presentation might in turn be used to issue a new request, for example, if the user were to click on the map. The procedures will be probably repeated many times during a geospatial query session. The detailed workflow of how an 
internet mapping application works can be illustrated by Figure 2.4 .2 below.

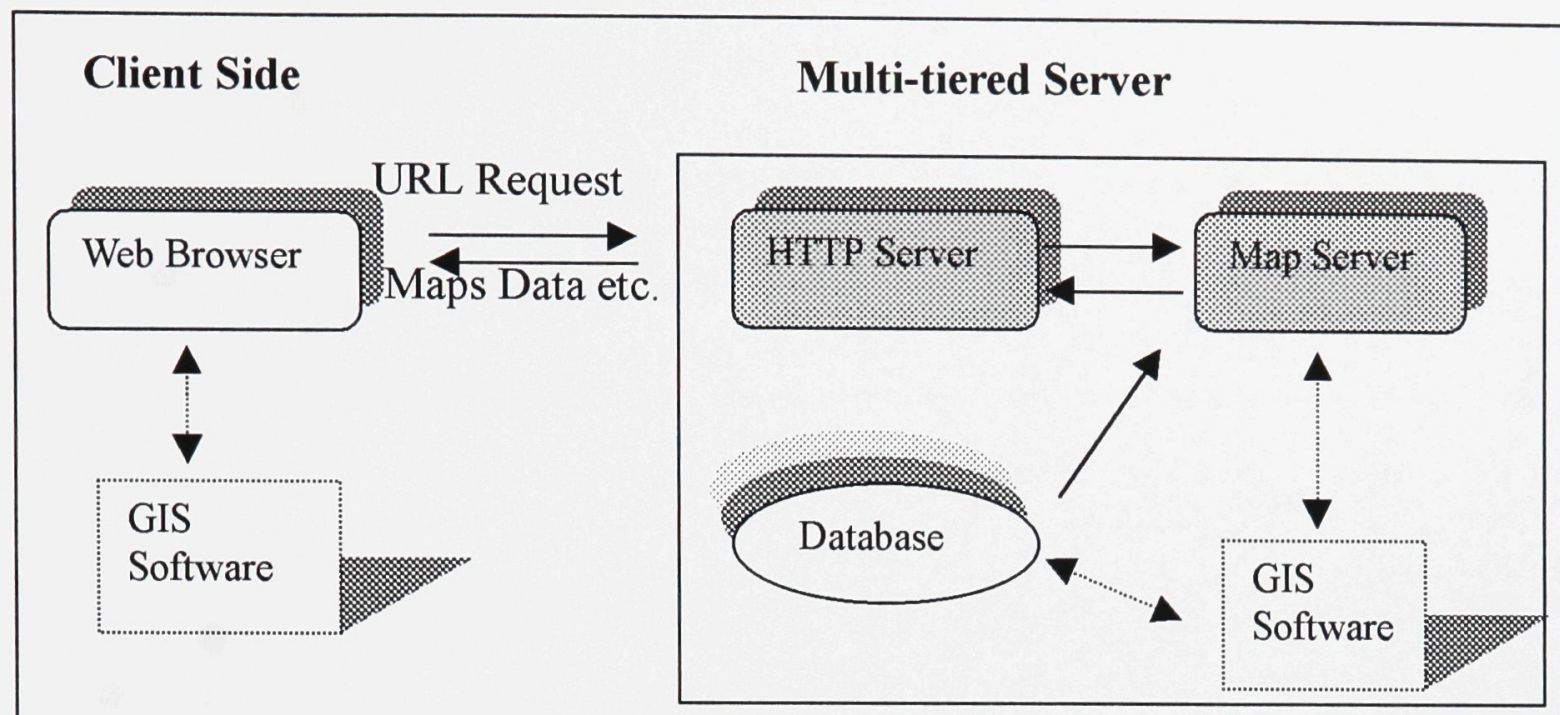

Figure 2.4.2 Workflow Chart of A Web Mapping Application

In many cases, the architecture of a site may differ from this general model mentioned above. Often that the web mapping software (WebGIS) incorporates data reading, mapping, and basic geospatial analysis capabilities, which eliminates the need for additional GIS software. On the other hand, with rapidly changing technology, Internet mapping techniques and WebGIS will become more powerful. What is not available now will soon become possible tomorrow. Therefore, it is likely that WebGIS will include more GIS functions and will process and analyze spatial data directly by itself in the future. Generally speaking, it may appear less likely that an independent GIS system will be linked to a Map server or WebGIS CGI program in the future. It is more likely that the multi-tier server, within the architecture of Figure 2.4.2, will be simplified like the one in the general model, figure 2.4 .1 , to satisfy most of the common needs of spatial information processing. 


\subsubsection{Issues for Web Mapping Design}

To develop a successful map-based Web application, similar procedures must be followed as those for creating successful Internet sites of other types. It is suggested by Cammack (1999, P157) that five main issues need to be addressed to create interactive maps on the Web. The five issues are as follows:

Site Configuration: This is to determine the primary interactive process location with respect to the basic client/server model introduced above. It includes client-based mainframe, server-based processing model and hybrid strategies that combine both server and client processes to optimize performance and meet special user needs.

Site Format: This refers to site format decision whether single pages, multiple pages, or frames are to be used.

Web Interface: Interface with the two primary web browsers, Netscape Navigator and Internet Explorer, requires additional plug-ins beside the standard HTML and DHTML languages as both lack spatial data handling capability. Generally, either -Macromedia Shockwave or Java, JavaScript, VB script, PHP script has to be added as a complement to HTML or DHTML.

Web Data types: HTML, DHTML and Java are the most common Web data formats. But, other data types, such as, streaming and VRML are also very valuable tools for 
interactive mapping development.

Content Interface: There are two categories of content Interface - customized and generic, which are designed for specific single products and multiple products respectively.

Although many aspects need to be considered in creating Interactive maps via the Web, the determination of a proper site configuration is considered the most fundamental element in developing Web based mapping applications. This study will focus on exploring implementing site configuration strategies for developing interactive map products and services online.

\subsubsection{Strategies for Putting Maps Online}

The first decision to be made for designing a Web mapping strategy is where the map interaction should be located: Client, Web Server, or Map Server. Thus, three basic types of site configuration strategies: server side strategies, client side strategies and hybrid strategies exist (Cammack, 1999). As their names suggest server-side strategies allow users to submit requests for data and analysis from a client to a Web server. The Web server is responsible for processing the requests and returns data or a solution to the remote client. Client-side strategies allow users to perform some data manipulation and analysis locally on their own machines. Server and client processes can be combined into hybrid strategies that optimize performance and better meet specific user requirements. 


\section{- Server Side Strategies}

This approach focuses on providing GIS data and analysis "on demand" from a primary or major server that has accessed both the data and the software needed to process this data. This strategy is comparable to the traditional 'terminal to mainframe' models for running GIS on local networks. Minimal processing power is required of the client (it is a "dumb" terminal in the traditional model). The client only needs the ability to submit requests and display responses. The map server is often applied for this type of site configuration. Users' requests for maps are "served" by a host from the server side.

Figure 2.4.3 below shows the Server Side Strategy.

The general procedures are:

1. A user sends requests from a Web browser to a server across the Internet.

2. An HTTP server processes the requests, renders the spatial data and analysis request to the Map Server (here, CGI script is used).

3. The Map Server sends result back to the HTTP server.

4. The response is returned to the user to be viewed using a Web browser.

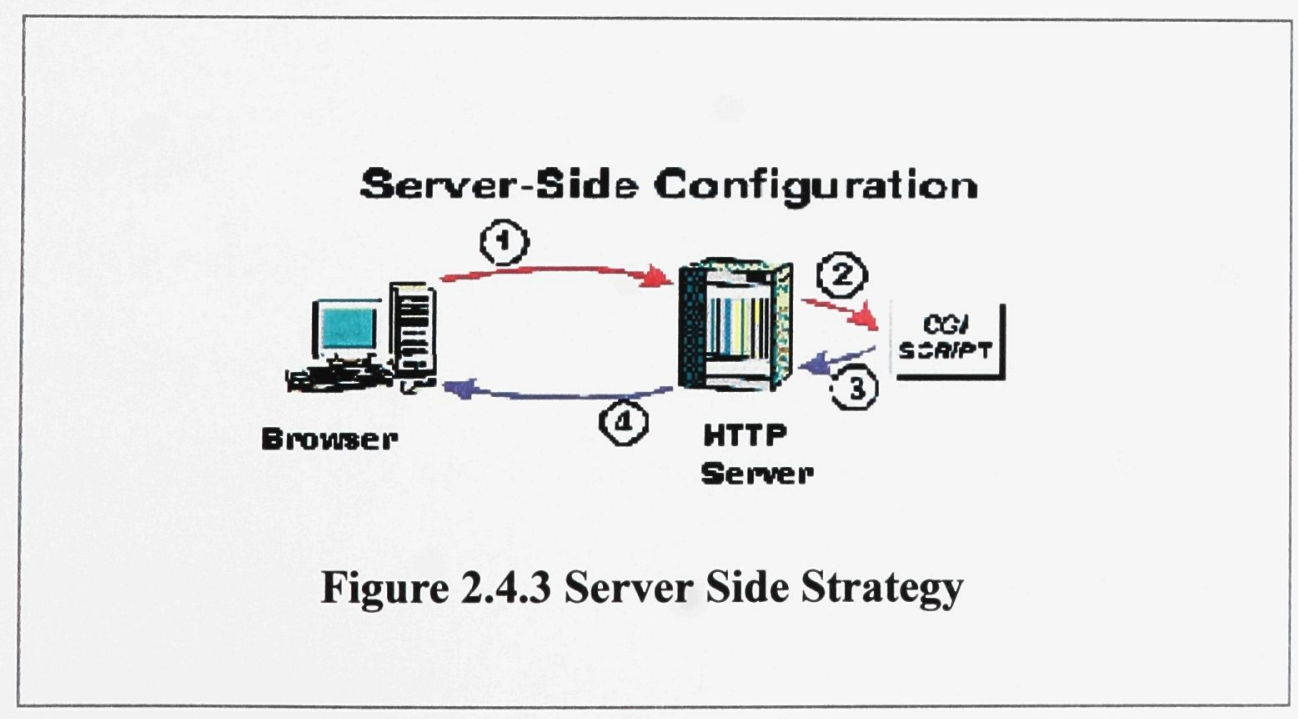


From the above illustrations, we can see that server side spatial data processing strategy relies heavily on the ability of users to send requests to the Map server (CGI script here) application tied in the Web server. CGI script is usually written in one of widely used languages such as Perl, $\mathrm{C}++$ and Visual Basic or can be purchased from commercial Web mapping product vendor directly, such as ESRI's ArcIMS, or Autodesk's MapGuide.

\section{Advantages}

The advantages of server side strategies include that they allow users to access or process large and complex datasets by high-performance servers that would be difficult to transfer across the Internet or process locally on a client's platform. A high-performance server allows complex GIS analytical routines to be run quickly even by clients who have no easy access to sophisticated hardware and GIS software. More important to use a server side strategy is that it allows more control to be exerted over what the user is permitted to do with the data, and perhaps, will also be helpful in ensuring that spatial data is used in a more correct way.

\section{Disadvantages}

For a server side design strategy, every request--no matter how small it is--must be returned and processed by that server. The responses must then be returned to the client across the Internet. The performance will be greatly affected by the bandwidth and network traffic on the Internet between the server and client, particularly, when responses involve transferring large files. The Web geospatial processing does not take advantage of 
the processing power of the user's own "client" computer, which is used merely to submit a request and display the response.

Generally, server-side strategy design is considered to be suitable for mass market applications with thousands or millions of users having little need for GIS analysis capabilities. (Plewe 1997)

\section{- Client Side Strategies}

Client-side applications attempt to shift some of the work of processing requests to the user's computer, sometimes referred to as a thick client approach. Instead of forcing the server to do most of the work, some of the spatial processing capabilities are downloaded to the client's platform, or reside there, and data is processed locally. Figure 2.4 .4 shows a client side strategy.

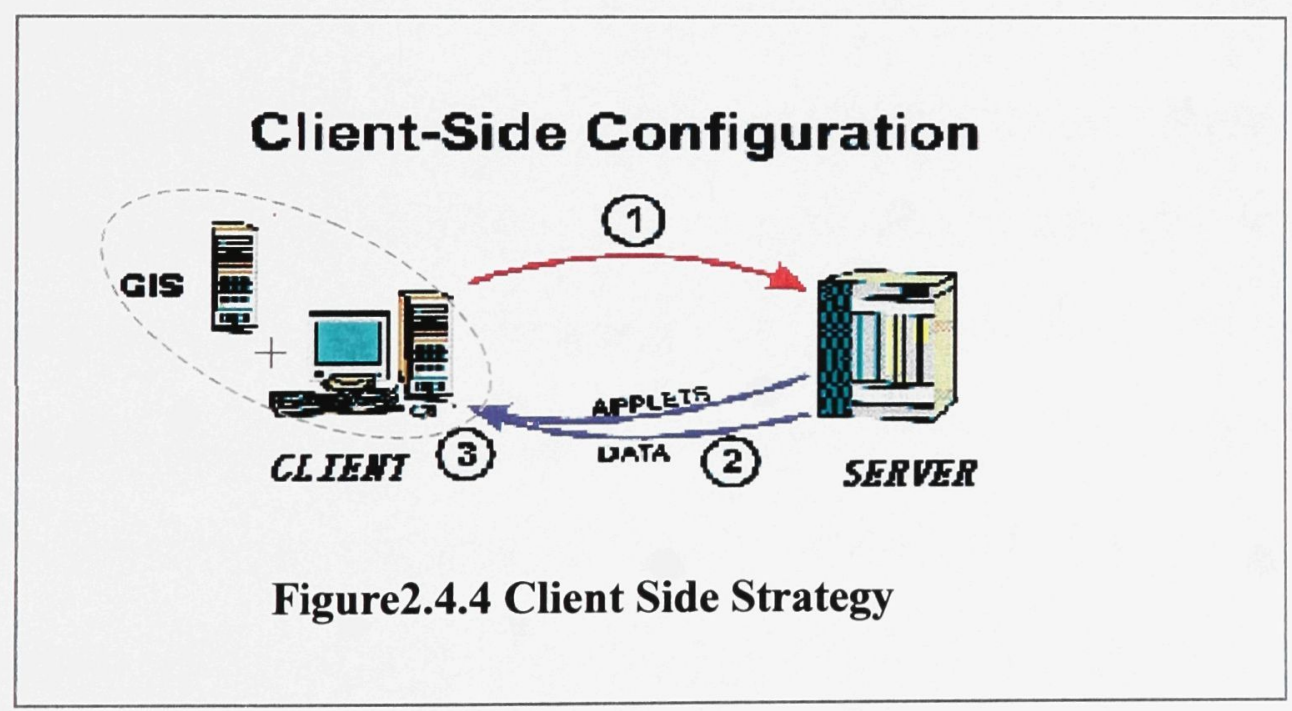

The general workflows are as follows:

1. a client send requests to the server, 
2. the server processes the request and returns data together with applets and plugins as needed,

3. data processed locally by the client's computer.

\section{Advantages}

Client side web mapping application design takes advantage of the processing power of the user's own computer and relieves the server's work load. The user can be given greater control of the data analysis process which is more flexible for meeting users' specific needs. Once the server has delivered its response, the user can work with the data without having to send and receive messages across the Internet. This will greatly reduce the limitations inherent in Internet traffic.

\section{Disadvantages}

The response from the server can involve the transfer of large amounts of data as well as applets, which can cause delays. Also, there are more limitations by the power limitations of client machine and the geographic knowledge of the user. The large and complex datasets may be hard to process or run more slowly on the client platform if it is not a very powerful one. Complex GIS analytical routines also require very powerful client platform. Due to lack of the geographic and cartographic knowledge, users may not have the training needed to employ the data and analysis functions properly. Client side strategy works well for services used by a smaller set of GIS-savvy users within an Intranet or a high speed Internet. 


\section{- Combination and Hybrid Strategies}

As described above, both pure server-side and client-side strategies have distinct limitations. If server-side strategies involve frequent transfers, their performance is sensitive to Internet bandwidth and network traffic. Moreover, client-side strategies are sensitive to the computational power of the client, meaning some tasks may run slowly when there is mismatch between processing demands and processor power.

Server-side and client-side strategies can be combined to produce hybrid solutions that better match to the capabilities of both server and client. Tasks that involve heavy database use or complex analyses can be assigned to the faster machine, typically, the server. Those tasks that require greater control by the user can be assigned to the client. Then, both the client and server share some information about their power and capabilities so data and applets can be assigned to each of them to maximize performance. A hybrid client-server framework is shown in figure 2.4.5.

\section{Hybrid Client-Server Combination}

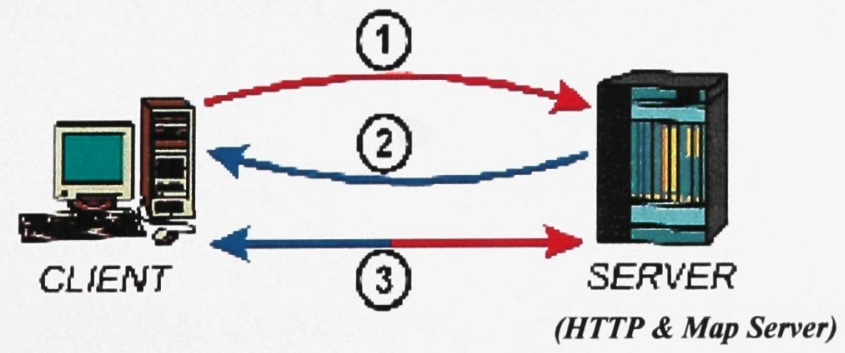

Figure 2.4.5 Hybrid Client/Server Strategy

The general procedures of hybrid strategy are: 
1. A user makes a request for data.

2. Some data and applets are returned based on specific request.

3. Continued interaction and data transfer takes place between Server and Client.

Hybrid solutions are especially useful for certain "pay-for-use" and "maps on demand" applications where users would "subscribe" to make periodic or regular use of a server for data or specific types of analysis. (Waters, 2001) Designing an effective hybrid solution is the most efficient, but, it requires the designer to understand in detail about the audience for the Web mapping application, including the capabilities of the audience's machines and the audience's knowledge of GIS skills.

\subsection{Conclusion}

The three fundamental web mapping application strategies described have already been employed to provide geospatial information and GIS functionality through the Internet in many cases. Although, site configuration is usually less of a choice and more an acceptance of what is available to the project designer, it is important to know the Internet structure and understand how to choose the proper site configuration strategy for a specific project so as to set up a successful Web mapping application (Cammack, 1999).

Some major Web mapping applications which represent different types of purpose and several typical WebGIS systems will be taken as examples and discussed in detail in the next chapter. 


\section{CHAPTER 3. CURRENT WEBGIS SYSTEMS AND WEB MAPPING APPLICATIONS}

\subsection{Introduction}

The pace of innovation has increased in the new Millennium. Everything appears to be rapidly changing. Those who use the Web to provide or obtain spatial data and spatial processing are becoming involved in this spatial Web's growth. As for Web mapping, there are more vendors, more products and more capabilities. With a broad look at today's Web mapping phenomenon, it is noticeable that the list of Web mapping sites through the GeoWorld Website

(http://www.geoplace.com/gw/2001/201/webmapsites.asp) grows longer every month (over 300 by May, 2002).

To help sort through the various options, it would be helpful to know what are the current WebGIS systems and the available Web mapping applications, for creating online mapping and analysis services.

Hereafter, several popular WebGIS systems and different types of Web mapping applications will be introduced to examine the current situation and the existing trends in Web Mapping technology.

\subsection{WebGIS Systems}

Most Web mapping solutions use middleware programs to launch GIS capabilities on the World Wide Web. Therefore, it is necessary to know the existing middleware - WebGIS 
software, both off-the-shelf and open sources, and think about which approach would be suitable for specific circumstances.

WebGIS or Web mapping software is delivered mainly via commercial companies, though there are also some outstanding open sources available. We see that almost all of the "old" geoprocessing software vendors are now on the list of Web mapping software vendors. For example, Autodesk has MapGuide, Bentley has ModelServer Discovery, the most popular ESRI company has its third generation WEBGIS: ArcIMS, GE Smallworld also owns an Internet application server, and Intergraph's GeoMedia Web Server, etc. The freeware includes Minnesota University's MapServer, GeoBean by the Chinese Academy of Science, etc.

With the large numbers of existing WebGIS or Web Mapping software, it would be nearly impossible to discuss all of them and directly compare them without understanding and using them thoroughly. Thus, only the major commercial and freeware products have been chosen from the many Web mapping products to explore the current status of spatial Web technology.

\subsubsection{Commercial}

Among today's Internet geospatial solution providers, Autodesk's MapGuide, ESRI's MapObjects IMS, ArcView IMS, ArcIMS, Intergraph's GeoMedia WebServer and MapInfo's ProServer are discussed in this section. 
- AUTODESK

Website: http://www.autodesk.com

Software Name: MapGuide

Autodesk MapGuide Web mapping system delivers maps and data to the Internet or intranet, enabling people to access and interact with live spatial information. By adding value to spatial data, MapGuide empowers users with capabilities to develop, manage, and distribute GIS and design applications on the Internet or your Intranet. The current version of MapGuide is version 6.

MapGuide provides an object-based approach to map browsing. It encompasses three products: MapGuide Viewer, MapGuide Author and MapGuide Server. Autodesk MapGuide Viewer has three versions: Active X Control, Plug-In, and Java ${ }^{\mathrm{TM}}$ Edition, each requires different configurations. MapGuide Author is used to create Webpublishable maps. The product allows users to create a file from existing spatial data that meet Map Window File (MWF) specifications. The MWF file is created using methods much like other map composition tools. Users select layers, areas of interest and cartographic conventions. Developers can select different graphic styles or turn map elements on and off as the user zooms in or out. Databases can be linked to map elements so that a click on a location can serve as the basis for a database query that returns information such as the demographics of a census location.

In the MapGuide arena, map elements are individual objects. The resulting map that is distributed provides a high level of interaction. This is due to the MapGuide plug-in, 
which is about 1-3 megabytes in size depending on platform and can be downloaded freely. Typically, browsers with plug-ins have more local capabilities without requiring to be triggered by the server.

The MapGuide Server component is a Web server application that sends out MWF files on request. It also allows MWF files to send back requests for data stored in a database and can return that data to the browser as a table or text.

MapGuide Server is multi-threaded, and can process several requests at once. It has security capabilities for restricting access to certain themes. In general, a threaded solution with a small footprint and small additional user demand makes MapGuide more scalable (i.e., to handle increasing load) than the solution that is not threaded.

Because MapGuide has its own specific plug-in solution, it allows the browser to perform many actions locally. As the mouse is moved over maps, individual elements (e.g., parcels, road segments, etc.) can be highlighted and selected with a mouse click. Users can zoom in and out and pan over the image, as well as "zoom go to," which is a combination of locator and zoom operations.

MapGuide Author is a standalone package with an interface that's similar to other Autodesk GIS/desktop mapping software. It uses geographic data in Autodesk's Spatial Data File format. Author comes with conversion facilities for ASCII-formatted files, Atlas BNA, MapInfo MIF and ArcView shapefiles. With these files as input, the map is 
created and saved in MWF format. A key aspect of the MWF structure is that Structured Query Language (SQL) operations can be associated with map elements such as polygons representing census blocks. The MWF map would be sent to a browser using MapGuide Server and viewed locally within the Web browser using the MapGuide plug-in. By using MapGuide locally, the user can display the map and select one (or more) graphic elements with the mouse. The codes for the selected elements would be returned by the MapGuide plug-in to the server, which would pass the selected values to an accessible online database. MapGuide Server supports ODBC-compliant SQL servers. The data would be sent to the browser as a Hypertext Markup Language (HTML)-formatted table or as text. A key point in this process is that the MapGuide map's characteristics have been pre-defined in the map production process. The user can interact with the map in several complex, predefined ways, but the map isn't created dynamically in response to user input. If the user wanted to view another area, then a different MWF file would need to be retrieved and sent to the browser. At the same time, however, dynamic queries can be developed from the map objects and submitted to a database for dynamic database reports.

MapGuide Author has a full set of map composition functionality in a standard Windows structure. It is a tool to help user design online maps to be generated from the database. Except for the requirements for linking Web operations to map locations and other similar Web-related functions, it is as easy to use as any map composition software. 
System Requirements:

For MapGuide Viewer: three versions of the viewer require different configurations. Generally, either Netscape Navigator or Microsoft Internet Explorer can be used by each version.

For MapGuide Author: Microsoft Windows98 Version 2, Windows NT 4.0 with Service Pack 4 or later versions, or Windows 2000 Professional.

MapGuide Server requires the following OS Envirnment:

- Microsoft Windows NT 4.0 Server with Service Pack 4 or a later version with one of the following:

Microsoft Internet Information Server 4.0

Netscape Enterprise Server 3.6 with Service Pack 3

iPlanet $^{\mathrm{TM}}$ Web Server Enterprise 4.1 with Service Pack 8

- Microsoft Windows 2000 Server, Service Pack 2 or later with Microsoft Internet Information Server 5.0

\section{- ESRI}

\section{URL: http://www.esri.com}

Software Name: MapObject Internet Map Server; MapObject Internet Map Server; ArcIMS

\section{ESRI MapObjects IMS}

MapObject Internet Map Server (IMS) is an extension to the MapObjects package. This collection of components can be used to build a wide range of dynamic mapping and GIS 
applications, using any functionality in MapObjects (which includes a large part of the functionality of Arc/Info.)

MapObjects is a suite of Windows mapping and GIS components that consists of about 35 ActiveX objects. Along with a programming language such as Visual Basic, programmers can use MapObjects to create customized mapping and/or GIS applications. The overall structure of MapObject IMS is similar to that of MapInfo's ProServer. IMS uses programming software that accepts requests from a Web browser and forwards them to a MapObjects program. MapObjects itself is a programming environment in which different operations can be performed. MapObjects supports map composition, address matching, spatial queries, links to databases, etc. In effect, the IMS allows a Web browser to be the user interface to a MapObjects application. No tools are provided to develop either the browser or server applications, and the programmer must be proficient in a programming language and HTML. The IMS package can manage multiple instances of MapObjects, so multiple users can access the Web site. Like MapInfo ProServer, IMS requires no plug-in for the browser.

With the MapObject IMS package, ESRI includes a Java (and ActiveX) applet called MapCafe, which creates a downloadable mapping interface. When a Web browser accesses a site with MapCafe, the MapCafe application is downloaded to the browser, assuming that the browser and platform are Java or ActiveX capable. MapCafe adds useful capabilities to the browser and provides local zoom, pan and query capabilities. 
The source code for the applet is included so the functionality can be expanded by the developer who has the capability of programming.

MapObjects is focusing on supporting the developers who need to build GIS application in MapObjects using Visual Basic, Visual C++, Delphi, Borland C++ Builder, Visual FoxPro, and Power Builder. These developing facilities are provided in MapObjects IMS.

System Requirements: Any browser supporting standard HTML. Microsoft Internet Information Server or Netscape Server running under Windows NT, MapObjects and Visual Basic 4.0.

\section{ESRI ArcView Internet Map Server (ArcView IMS)}

ArcView IMS is an entirely different approach to the MapObjects IMS. ArcView IMS is designed to be a ready-to-use program, although it is less flexible than MapObjects IMS because the latter allows you to program any functionality you need.

When ArcView IMS forwards a request to ArcView, an avenue script is started, which has been written specifically to handle the limited range of applications you wish to support online. This script can be virtually identical to any other Avenue script, except that there is no GUI interface (which will be handled by HTML pages), and maps are drawn to a "virtual view window", which actually results in a raster image returned to the 
client. As a result, ArcView functionality can be accessed through Avenue scripting. ArcView IMS functions similarly as MapObject IMS. In order to improve the interface capabilities, a Java applet, Mapcafe can be used together with ArcView IMS. One significant difference between IMS for ArcView and IMS for MapObjects, however, is the level of programming skills needed to create a map and initial application. The IMS for ArcView product includes Web map composition capabilities similar to those of MapGuide. Windows-compliant dialog boxes and wizards make the application/map development task much easier. However, creating a complete Web mapping site still requires users to master significant technical complexities.

\section{ESRI ArcIMS}

ArcIMS 3.0 is the third generation Internet Map Server product from ESRI providing a common platform for exchanging and sharing GIS resources. It has the flavour of both desktop applications like ArcView IMS solution and developers choice of customising MapObjects IMS Internet GIS solutions. It can serve in diverse applications as ecommerce, location based services, Enterprise Resource Planning (ERP) etc. ArcIMS features a more open and interoperable environment, easy-to-use framework, and highly scalable architecture. It has replaced both MapObjects IMS and ArcView IMS products.

\section{- INTERGRAPH}

URL: http://www.intergraph com/gis

Software name: GeoMedia Web Map 
Intergraph's GeoMedia WebMap is a Web-based visualization tool offering real-time links to GIS data warehouses. One can query a database and see information in the form of a map. GeoMedia Web Map software retrieves data from Intergraph's GIS and automated mapping/ facilities management products (Modular GIS Environment, FRAMME and MGEDM) as well as Exton, Pa.-based Bentley Systems Inc.'s MicroStation design files. GeoMedia does not require these packages to run, but accesses the data files associated with them. The geographic data are converted to Computer Graphics Metafile (CGM) format and sent to the Internet. CGM files from GeoMedia Web Map or any other source can be viewed on the Web using the ActiveCGM plug-in, which is available free from InterCAP Graphics Systems (Web: http://www.intercap.com). The ActiveCGM plug-in works with Microsoft Explorer and Netscape Navigator with Windows 98 or NT/2000, but is not available for UNIX- or Macintosh-based browsers.

The ActiveCGM plug-in allows graphic elements to be distributed as objects and manipulated by the browser. The ability to pan and zoom locally is supported, as is the ability to highlight individual objects and access their keys. The structure makes it easy to link map graphics and a list of items in table format. For example, one part of the browser can display a list of stores in an area. If a specific store is selected, the graphics can zoom to that location and highlight the store footprint. Conversely, users can click on a specific street and the browser's text portion will display information on the street, such as pavement type. In both examples, the browser (and plug-in or ActiveX control) can identify the graphic element selected and return its key to the server, which then can 
update the text from the database. Selecting a text item reverses the process by sending the key to the server, which generates a new CGM and sends it to the browser

GeoMedia accesses directly supported graphics files (or uses copies if security is an issue). A map definition file is created to determine how the various graphic elements will be displayed in CGM format. In the map definition file, entries indicate characteristics such as line and polygon fill styles and the map scales at which various feature classes can be displayed, as well as the locations and actions to be taken at "hot spots" and the sensitivity that various objects will have in order to be selected by mouse operations on the browser.

To implement a GeoMedia Web Map server, it is necessary to enclose the appropriate GeoMedia Web Map commands in a scripting structure such as Visual Basic. The overall script manages the interaction with the browser and the database and controls how the text portions of the browser are managed. GeoMedia Web Map manages the conversion of the necessary graphics to CGM format and manages the links between the graphic elements and the corresponding database records

System Requirements: InterCAP Graphic Systems' ActiveCGM plug-in with Netscape Navigator 2.0 or later versions running on Windows 98 or, Windows NT/2000, ActiveCGM control with Microsoft Explorer 3.0 or later versions. The plug-in is not available on UNIX- or Macintosh-based browsers. It also requires Microsoft Windows 
NT with Internet Information Server. ODBC-compliant database. Scripting environment, e.g., Visual Basic, etc.

The outstanding point is that Geomedia Web Server is the only Web-based map visualization tool that links in real time to one or more GIS data warehouses. You can build Web-based mapping applications that combine, analyze, and distribute GIS information from multiple sources across intranet or the Internet. GeoMedia WebEnterprise enables you to analyze and manipulate geographic data on the Web. Companies can create custom Web mapping applications that are dynamic, open and scalable. New features in version 4.0 expand the Web-based GIS capabilities in both products.

\section{- MAPINFO}

URL: http://www.mapinfo.com

Software name: MapInfo ProServer

MapInfo ProServer is a Web server application that, linked with MapInfo and MapBasic, provides a complete environment for dynamically creating and serving MapInfo maps and data. MapInfo 4. $\mathrm{x}$ is a widely used desktop mapping/GIS package. MapBasic is a rich programming language through which MapInfo operations can be accessed. It is also possible to develop complex standalone applications using MapBasic, which calls functions from MapInfo. 
MapInfo ProServer provides a gateway through which commands sent from a browser are processed and sent as input to a MapInfo "instance," which is a running MapInfo program. Perhaps the best way for users to think of the package is to consider the browser as a way to provide input to a MapBasic application. MapInfo ProServer provides a programming environment in which a developer proficient in MapBasic and Web programming can create an application that responds to the user's input, performs MapInfo operations--which could include creating a map graphic--and returns the results in standard HTML-supported structures. The map that is created would be saved in a Web-compatible format such as JPEG and downloaded to the browser without plug-in or client-side code. Maps are not prepublished but can be created on-the-fly in response to user input. Through the MapInfo ProServer interface, MapBasic and MapInfo operations can be retrieved, including spatial operations such as "search for points within a 5-mile radius of the point."

To use MapInfo ProServer for an application, the developer must be knowledgeable about MapBasic, HTML and codes that can allow the two to interact--typically programming software such as Visual Basic. Creating an application would involve the following process: an initial Web page created, using HTML, would be sent to the browser when the server is first accessed. This page could have any capabilities supported by HTML. For example, the user might be asked to enter a city and street address, and the information would be sent by the browser as a simple text string to the programming software on the server, which would process it and forward the appropriate commands to MapInfo ProServer, MapInfo and MapBasic. The MapBasic program 
would intercept the command and cause MapInfo to perform the appropriate action. In this instance, that would mean accessing the spatial data for the city and address and creating a map of the area. The MapInfo operations that convert an address to a geographic location also would be run, as would connection to a database with the geographic coordinates of, for example, all automated teller machines (ATMs).

The MapBasic program also would retrieve another MapInfo operation that could add symbols for those machines located within the search radius. Predefined graphic styles and labels would be applied by another part of the program. Finally, the map would be output in JPEG form and sent with some text to the client with the request "Click on the desired ATM machine." The user would click on the location and the browser would return the "x" and "y" coordinates of the click. These coordinates would be sent back to the server, then to MapInfo, which would identify the ATM at that location, access a database of information about the ATM and send it back as HTML text to the browser.

The key factor in the MapInfo structure is that MapInfo and MapBasic's full functionality is accessible to Web applications through MapInfo ProServer. MapInfo also has a powerful address matching package, MapMarker, that is accessible to MapInfo ProServer, so applications requiring address matching can be Web-based as well. MapInfo ProServer's access to MapBasic, MapInfo and MapMarker means that complex, dynamic operations are possible. It also means that developing programs to access these capabilities is a complex task which requires capable programmers. 
MapInfo recently announced an easy-to-use Web mapping product, MapXsite, but the product was not available in time to be included in this review. According to MapInfo, MapXsite primarily will focus on geocoding and serving resulting street or other location maps. It is designed for companies wanting to add maps of store locations or similar map information to their Web sites.

System Requirements: Any HTML-compatible browser. Windows NT/2000 or Windows 95/98 and a Web server that supports CGI. ODBC-compliant database or other data sources supported by MapInfo and MapBasic.

\subsubsection{Opensource Web Mapping Software}

\section{MapServer by University of Minesota}

URL: http://mapserver.gis.umn.edu

MapServer is an OpenSource development environment for building spatially enabled Internet applications. It was originally developed by the University of Minnesota (UMN) ForNet project in cooperation with NASA and the Minnesota Department of Natural Resources (MNDNR). Additional enhancement was made by the MNDNR and the Minnesota Land Management Information Center (LMIC). Current development is funded by the TerraSIP project, a NASA sponsored project between the UMN and 
consortium of land management interests. The current version of MapServer is version 3.5 .

The software builds upon other popular OpenSource or freeware systems like Shapelib, FreeType, Proj.4, libTIFF, Perl and others. It can run where most commercial systems will not or can not, on Linux/Apache platforms. MapServer is known to compile on most UNIXes and also can run under Windows NT/98/95.

The MapServer system now supports MapScript which allows popular scripting languages such as Perl, Python, Tk/Tcl, Guile and Java to access the MapServer C API. It provides a rich environment for developing applications that integrate disparate data. If the data has a spatial component and you can get to it via your favorite scripting enviroment then you can map it.

Although MapServer CGI application is not a full-featured GIS system, nor does it aspire to be, it provides a significant number of "out-of-the-box" functionalities to support a wide variety of web applications. For example, beyond browsing GIS data, MapServer allows you to create "geographic image maps", i.e. maps that can direct users to content. For the details of MapServer feature, Chapter 4 will introduce more by presenting a case study of online atlas application.

In short, to create an Internet GIS application and serve it on the net, primary factors such 
as the type of GIS functionality, downloading time into the client browser and the cost of the whole application are the essential considerations. With so many existing and rapidly changing Web mapping systems, it is important to know that their general usefulness and features in order to identify appropriate software, hardware and network infrastructure to ensure efficient Geospatial online implementation.

\subsection{Examples of Web Mapping applications}

As the spatial Web grows, there are more online specialized geospatial services available. And while these resources are becoming simpler to use, there are increasing potentials for extended capability and complexity in various application fields. What is possible with Web mapping technology?

Generally speaking, the Web mapping application broadly ranges from static map presentations to sophisticated GIS analysis functions such as providing the shortest or quickest driving directions. With many faces of Internet mapping applications, they can be categorized into the following groups according to their uses and owners' primary constructing purposes:

- Government owned National Spatial Data Infrastructure (NSDI)

- Scientific research by academic organizations

- Public service by local government agencies

- Marketing and profit oriented commercial sites, and others. 


\subsubsection{National Spatial Data Infrastructure (NSDI)}

With the spread of the Internet, we have witnessed an enormous increase in the amount of geodata available on the $\mathrm{Web}$. There has been a growing awareness of the need to share and integrate geodata resources and geodata production processes. Geospatial data sharing between government institutions has been difficult in practice, but with the advent of Web mapping technology, it is possible now.

The governments of many countries have mandated or at least encouraged putting the government produced spatial data online. All have led to arrive at a national (regional, sometimes) level of web based spatial data infrastructure (Elzakker, 2000) and made government owned spatial data much more accessible not only to the GIS community but also to the general public.

Starting with the United States, the President's "Executive Order 12906" calls for the establishment of the National Spatial Data Infrastructure defined as the technologies, policies, and the people necessary to promote sharing of geospatial data throughout all levels of government, the private and non-profit sectors, and the academic community. (http://www.fgdc.gov/nsdi).

The goal of this Infrastructure is to reduce duplication of efforts among agencies, improve quality and to make geographic data more accessible to the public, to increase the benefits of using available data, and to establish key partnerships with states, 
counties, cities, tribal nations, academia and the private sector to increase data availability.

The President's "Executive Order 12906" provides a base of practices and relationships among data producers and users that facilitate data sharing and use. This is a set of actions and new ways of accessing, sharing and using geographic data that enables far more comprehensive analysis of data to help decision-makers chose the best course(s) of action. Much has been accomplished in recent years to further the implementation of NSDI.

The Federal Geographic Data Committee (FGDC) of the United States is tasked by "Executive Order 12906" to develop procedures and assist in the implementation of a distributed discovery mechanism for digital geospatial data. Some outstanding examples of this type of Web based geospatial data service include the National Atlas of the United States (http://nationalatlas.gov/), National Geospatial Data Clearinghouse of the United States (http://www.fgdc.gov/clearinghouse), National Atlas of Canada (http://atlas.gc.ca/), etc

\section{National Atlas of the United States:}

URL: http://nationalatlas.gov/ 
A new version of National Atlas of the United States began in 1997. Like its predecessor, its goal is to promote greater national geographic awareness. This version delivers easy to use, map-like views of America's natural and socio-cultural landscapes. It is largely digital. The National Atlas is developed in conjunction with ESRI Company based on ArcIMS.

The National Atlas site includes products and services designed to stimulate the public to visualize and understand complex relationships between environments, places, and people. It contributes to the knowledge of the environmental, resource, demographic, economic, social, political, and historical dimensions of American life.

With a typical map, explore functions like zoom in/out, pan, identify, query etc. This Atlas serves the interests and needs of a diverse populace in many ways, including:

- as an essential reference,

- as a framework for information discovery,

- as an instrument of education,

- as an aid in research,

- and as an accurate and reliable source of government information.

It is designed to provide a reliable summary of national-scale geographic information and allow users to download and save into their own machine. Though it cannot provide extensively detailed map information, the Atlas can act as a map index to direct users to other linked sources for specific information. After surfing on its existing map functions, audience can find that the application design is easy to follow and the interface is very 
straightforward. Figure 3.3.1 shows the interface of National Atlas of the United States.

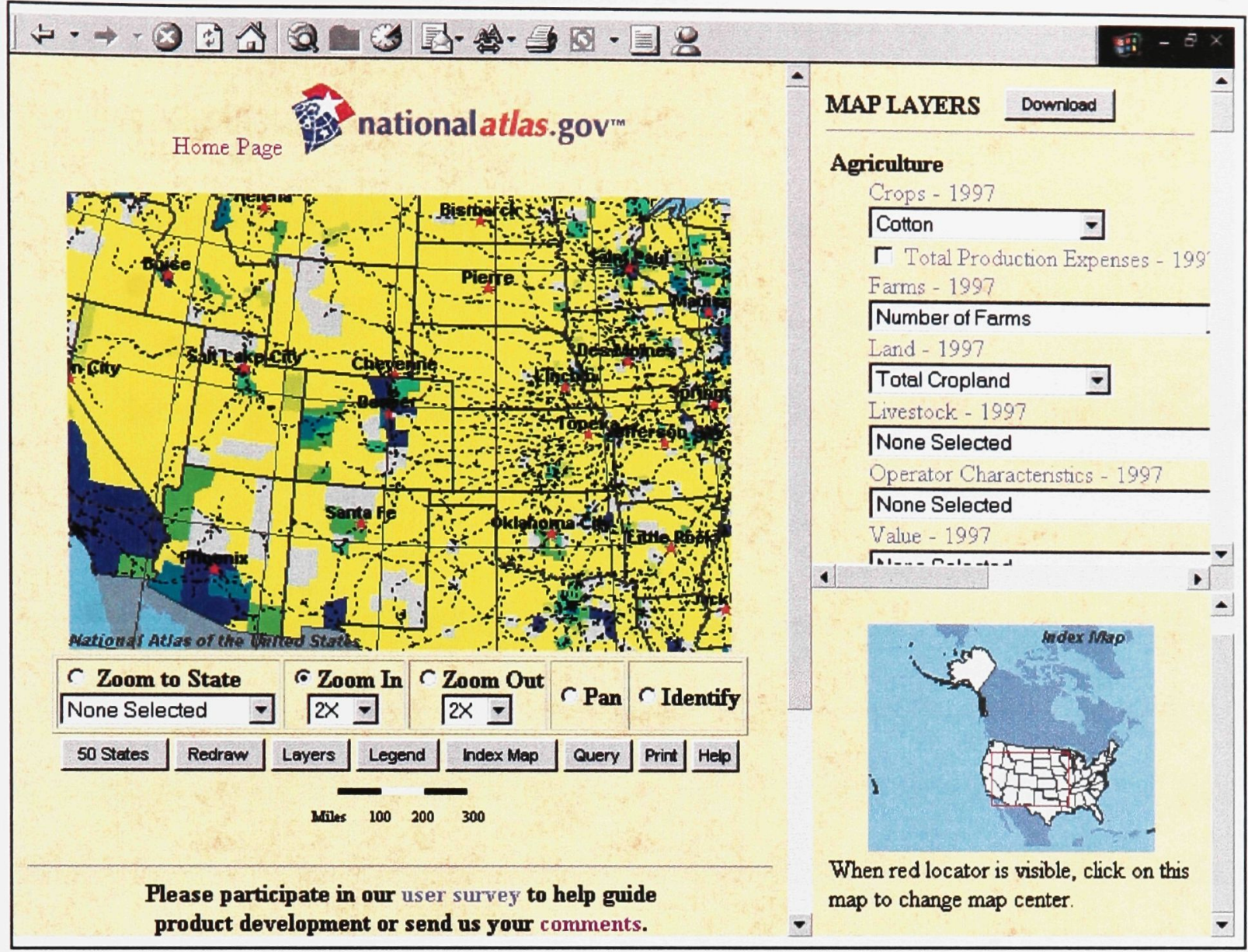

Figure 3.3.1 National Atlas of the United States

\section{National Atlas of Canada}

\section{URL: http://atlas.gc.ca/}

The current version of National Atlas of Canada is the $6^{\text {th }}$. Edition. The mandate of the Atlas is to provide a reliable summary of national-scale geographic information. It primarily provides visualization tools for high level, authoritative, information of national scope represented on maps. The sixth edition of the National Atlas of Canada has become a living electronic publication, with some of its products and services drawn from online databases. From a conceptual point of view, it is an information system that comprises an 
integrated, geospatial database accessible by means of specialized services and tools (Frappier,2000).

The Atlas was developed for three levels of users:

- the novice user who only wishes to view predefined maps,

- the advanced user who wants to chose their own layers, and

- the most advanced user who will define their own layers and query their information.

The functionality has continued to expand since the early Internet atlas. In addition to the choice of available map layers, the user now has access to the functions including: access to a metadata directory, editorial views of maps for each theme, zoom and pan, an identify function with attributes available for each geographic entity, a locator map that can be used for panning, tools for changing colors and potentially class intervals as well, and the option of saving customized maps. Although it cannot provide community-level information, the Atlas can direct users to organizations and online services, such as the Canadian Communities Atlas, which provides more detailed information at the local level. As well the Atlas links to CEONet and GeoGratis, the discovery and access components of the National Atlas Information Network.

The information portrayed in the National Atlas of Canada helps to explain the social, economic and bio-physical make-up of Canada and package the 
information in terms of issues and challenges that face Canada. The content of the atlas includes: Physical, Human, Economic, Historical geographic themes and some relevant Canadian issues such as Health, Climate Change,etc.

The current version using ESRI MapObjects Internet Mapping tools offers the ability to provide different interfaces for varying audiences with appropriate functionality for each user group. For example, sophisticated users may want to complete complex searches and be able to combine a wide variety of information to make their own unique maps. It is no longer a stand-alone paper product but has become a living database of integrated information of which an interactive publication on the web is its main manifestation. Figure 3.3.2 shows the basic interface of the National Atlas of Canada.

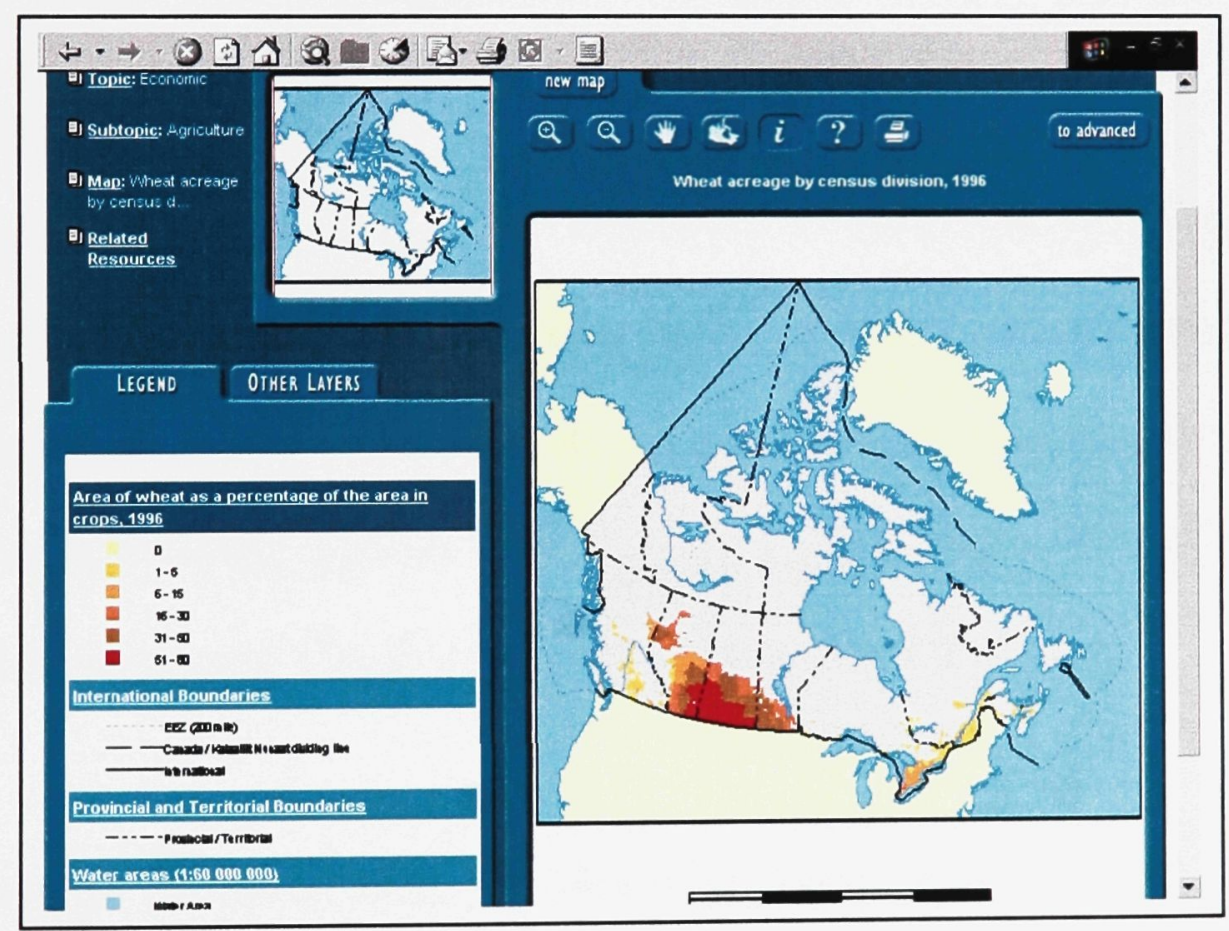

Figure 3.3.2 Basic Interface of the National Atlas of Canada

\subsubsection{Scientific Research:}

Nowadays, much of the scientific research of spatial and temporal feature exploration has 
conducted. Nonetheless, however precise or sophisticated the scientific research is, the data will be of limited value if it can't be shared and used efficiently in an easily accessible environment by scientists who need it.

Many geoscience organizations and scientists have increasingly realized the need for tools that allow them to share and publish geospatial data in a standard open environment. Many Web mapping applications for scientific research purposes have therefore been established and are running worldwide. The following is an example of online geospatial information example for the use of geoscience academic research USGS Antarctic Atlas.

\section{USGS Antarctic Atlas}

URL: Http://www.usgs.gov/antarctic

The U.S. Geological Survey (USGS) was established in 1879, principally involved in mineral exploration. Today's USGS expand the Survey's roles to include collecting and publishing geospatial data, performing extensive research on environmental issues far beyond its historical mining purview. The Antarctic Research Program is one of their ongoing tasks in conjunction with International Antarctic research groups.

Atlas of Antarctic Research began in December 1998. Antarctic Atlas has been developed using software from the National Atlas of the United States. It has been designed to promote greater geographic awareness of the continent and the digital geospatial data that describe it. It provides a common base for displaying research results 
and data collected, as well as descriptions of ongoing and past projects, when they become available for display. The Atlas is intended to serve the interests and needs of a diverse community as a reference, an information framework, an education tool, and a research aid. Its primary focus is as a tool for Antarctic researchers. It enables users to download and save data into local machines. The USGS provides ongoing development and maintenance of the Atlas through its Antarctic Program.

The Atlas of Antarctic Research site provides a full set of parameters used for display of the geographic data, including projection parameters used, layers existing, scales, data sources etc. It allows the viewer to browse the available Antarctic atlas and explore the maps using the provided function icons such as Zoom in/out, Pan, identify tool, turn on/off the map layers and so on, to redraw maps based on a specific configuration. By clicking the buttons, the request is passed to the server and the map can be interactively generated. As the user zooms in, the Atlas automatically replaces low-resolution layers with those of higher resolution and removes layers when the scale of display exceeds the resolution of the data. The interface is clear, quite functional and easy to follow. Figure 3.3.3 shows the content interface of the Atlas of Antarctic Research. 


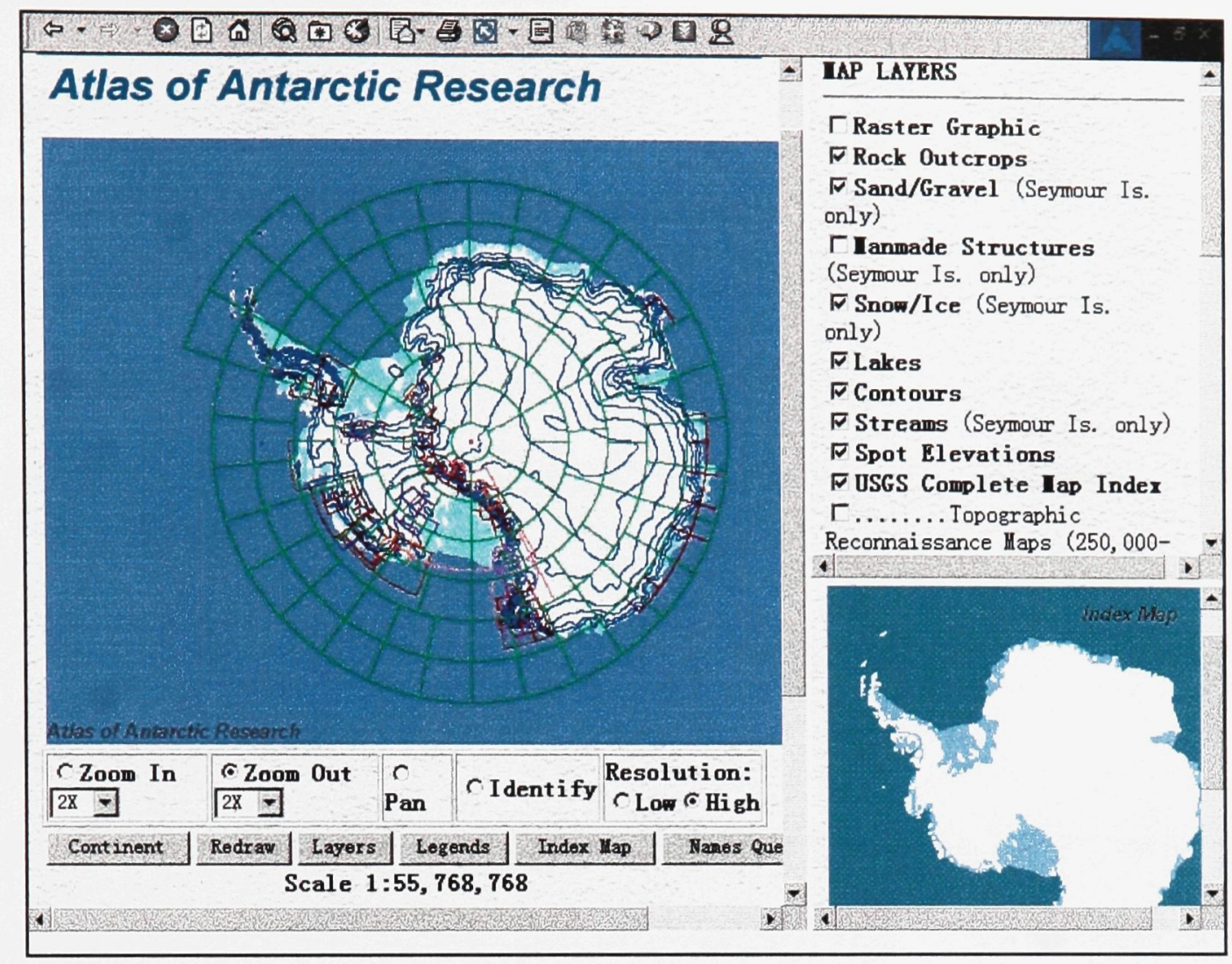

Figure 3.3.3 Interface of the Atlas of Antarctic Research

\subsubsection{Public Services}

A lot of Web based spatial information is dedicated to the regional public service by local government agencies or specific communities. Local government agencies, such as the City of Ottawa and Cabarrus County of North Carolina in the States, maintain their spatial information like local land use, road network, Gas, Water pipeline network system etc., and make it publicly available.

Two examples of public service mapping sites will be examined. 


\section{City of Ottawa}

URL: http://atlas.city.ottawa.on.ca/mapping/atlas/atlas.htm

The City of Ottawa has developed the "Atlas of Ottawa" web site to allow you to view and interact with linked databases and live maps that cover the entire City of Ottawa area. Figure 3.3.4 shows the "Atlas of Ottawa" Website Interface. Its content includes four parts:

- Interactive Maps

Interactive Maps allows user to interactively view and manipulate the live maps of Ottawa area, however, as this online mapping application was developed based on the Autodesk's MapGuide WebGIS system. It requires user downloading MapGuide browser plug-in in order to view the Ottawa atlas. As it will take some time to fill in the request form, download MapGuide viewer plug-in and install the plug-in, this usually discourages the general public audience from exploring the interactive atlas site.

- Air Photo

This is an air photo index which allows the user to check the availability of their wanted air photo for online shopping.

- General Use CD

The City of Ottawa has developed this web site for the general education purpose. It 
allows user to download a free GIS viewer package, ArcExplorer and download a sample of the City's databases, so the user can produce maps on their own computer.

- Picture Atlas

It contains a series of hyperlinked Ottawa map pictures in “.jpg" or ".html” format that allow users to view the static Ottawa maps.

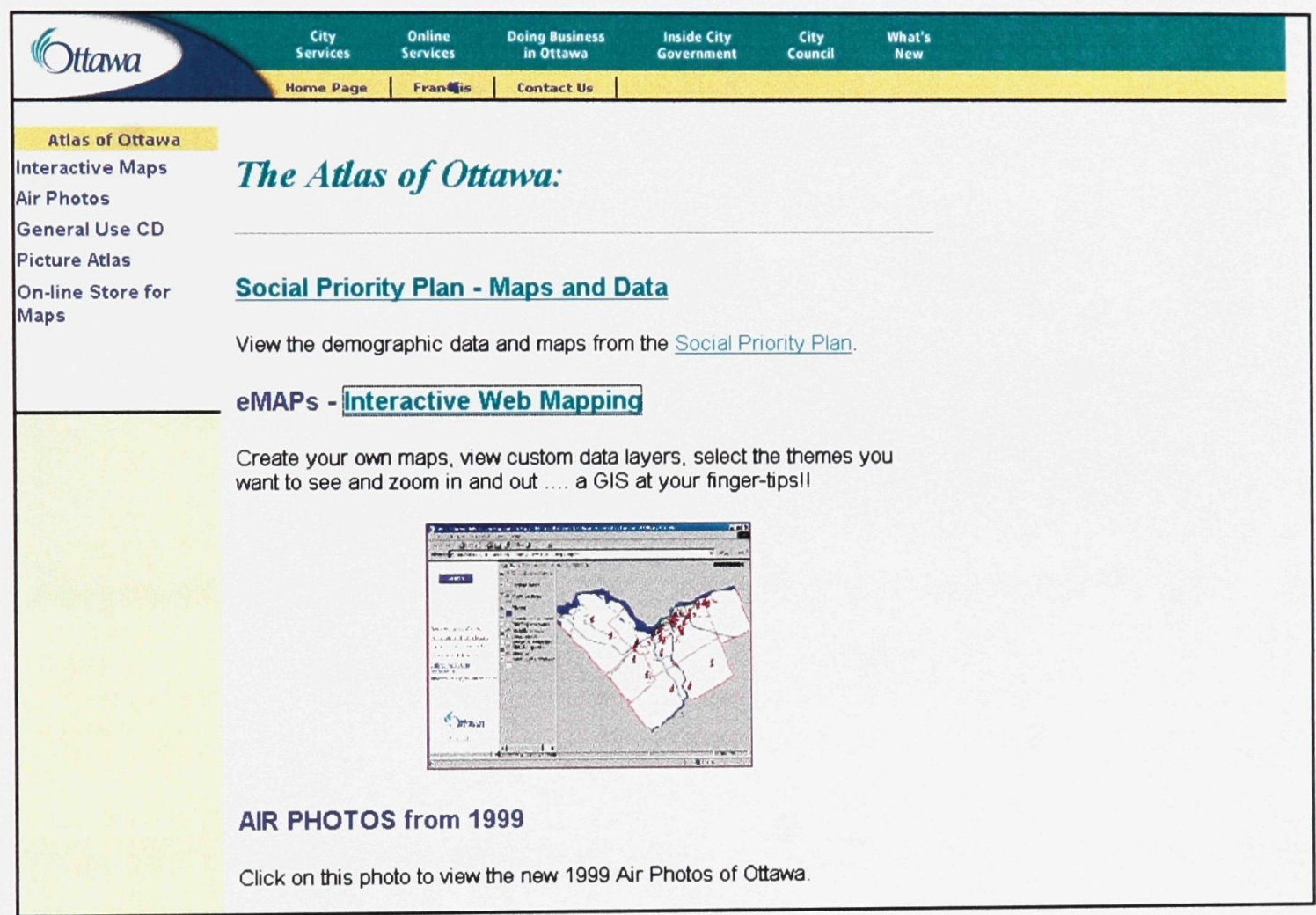

Figure 3.3.4 City of Ottawa- the Atlas of Ottawa Website Interface

\section{Cabarrus County}

URL: http://www.co.cabarrus.nc.us

The spatial data distribution on Cabarrus County government Website has been developed based on ESRI's MapObject Internet Map Server (MapObject IMS). It enables the local people to access the county's detailed land parcel records database and search 
for information such as, ownership, property value and taxes issues throughout the Web. The page of Cabarrus GIS application is shown in figure 3.3.5 Cabarrus County GIS Web Page.

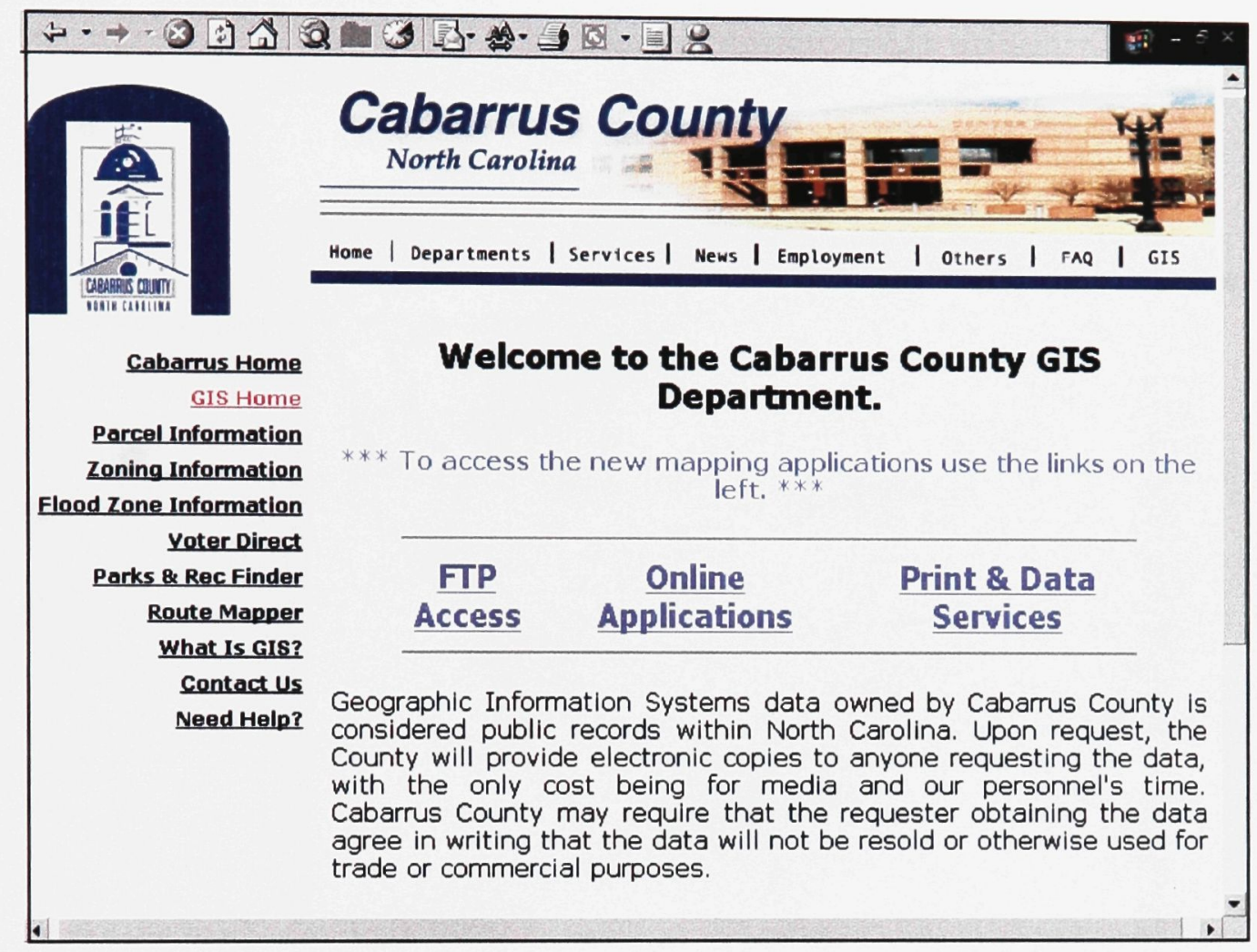

Figure 3.3.5 Cabarrus County GIS Web Page

With the idea of making the more frequently requested public information available, the county government developed a truly interactive site that allows taxpayers to connect with their tax -funded agencies and access the relevant land parcel database. The GIS Department of Cabarrus county has developed a Interactive Land information application based on MapObject IMS. Users who know the exactly address or parcel ID number can go directly to the land map and table for that parcel information. There are also zoom in/out, zoom to full extent, pan, and identify tool functions which allow users to locate the land parcel without having a specific address or ID number. The interface of the land 
information application of Cabarrus county is shown in figure 3.3.6. Land Information Application Interface, Cabarrus County.

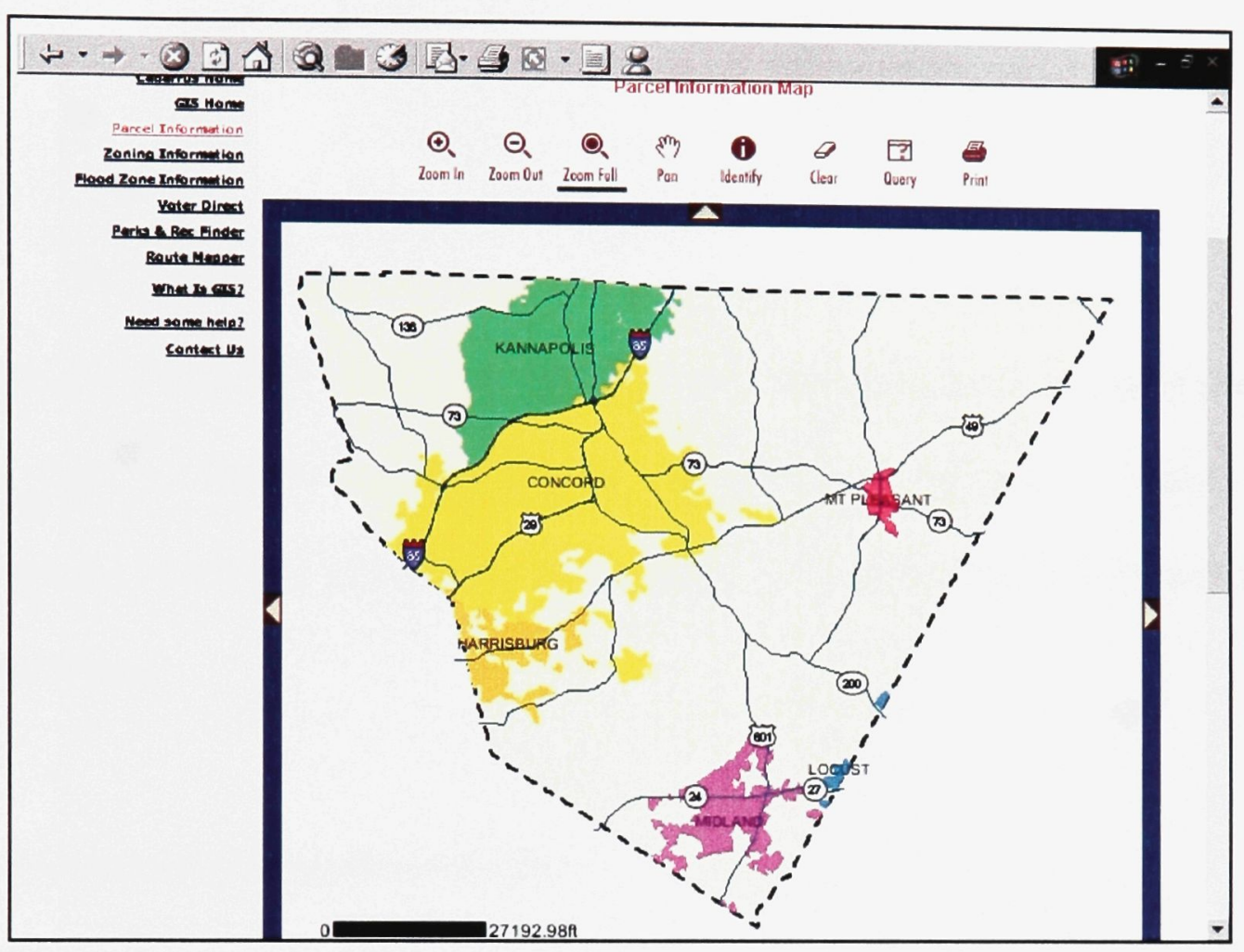

Figure 3.3.6 Land Information Application Interface, Cabarrus County

Once a required parcel has been selected, the application displays a map of that inquiry area, with the selected parcel outlined in green. Following the map, the map legend and a very detailed parcel information table (figure 3.3.7) are provided, including the owner's name, tax value, the last price the parcel was sold for, how it is zoned, and so on. 


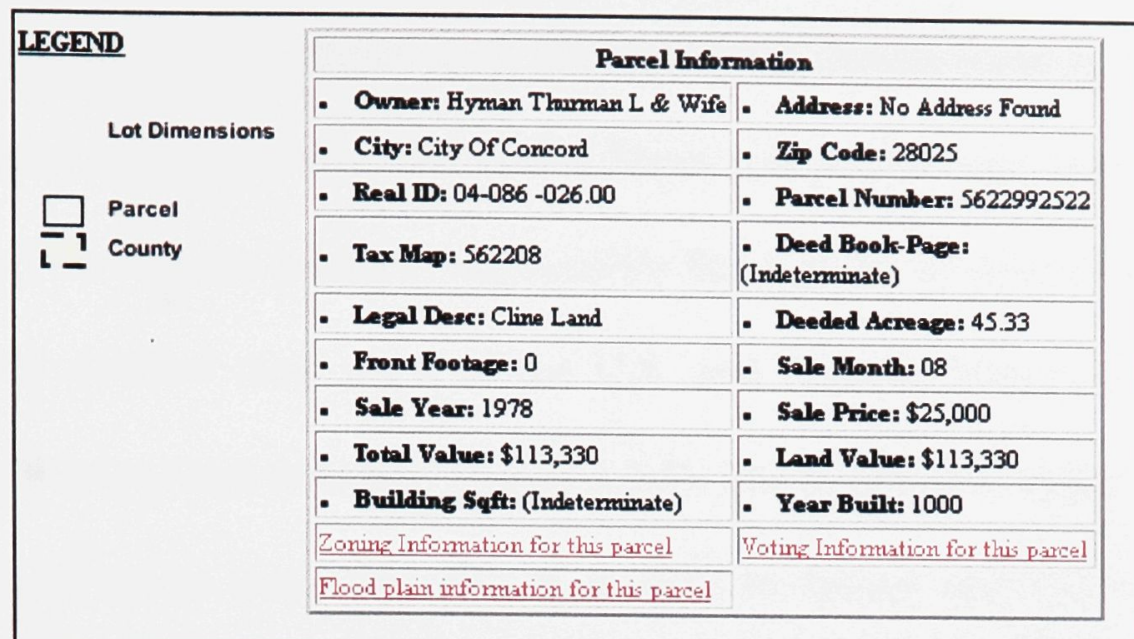

\section{Figure 3.3.7 Land Parcel Table of Cabarrus Land Information Application}

This type of Internet geospatial data application is especially useful for property appraisers, real estate agents, and local people who are involved in property disputes or tax problems.

\subsubsection{Commercial Application Sites}

Commercial types of web map applications refer to those initiated by a commercial company, that is profit or marketing orientated. Some examples are: MapQuest by Geosystems Global, MapBlast by Vicinity Corporation, or Realtor.Com by RealSelect, Inc., and the utility network applications such as water pipeline, electricity and TeleCom network management systems via Intranet or Internet.

\section{MapQuest by GeoSystems Global}

URL: http:// www.mapquest.com

The MapQuest Website is developed by Geosystems Global company. (It was sold to American Online at the beginning of 2001, so it belongs to AOL now.)

MapQuest provides services including generating maps of the world, providing driving 
directions in North America and European with fastest route or shortest route, giving basic road trip plans within the United States and yellow page searches within the United States. This site can generate high-quality Street maps of locations all over the world. It has very detailed road maps of the U.S. and Canada. However, the most impressive feature is its content interface (Figure 3.3.8). The interface is simple and straight-forward, making common users feel that it is easy to follow and understand. Many people, whether in the GIS field or not, often use these web mapping tool. The data sources of MapQuest are provided by Geosystems' own proprietary street database, Navtech, and other sources.

MapQuest map service allows users to save, print, download or email the resulting route maps. Users can also control the map size, color and coverage area, change icon label, and place location symbols onto a map. See website for more details. Figure 3.3.8 shows a query map result for Ottawa City.

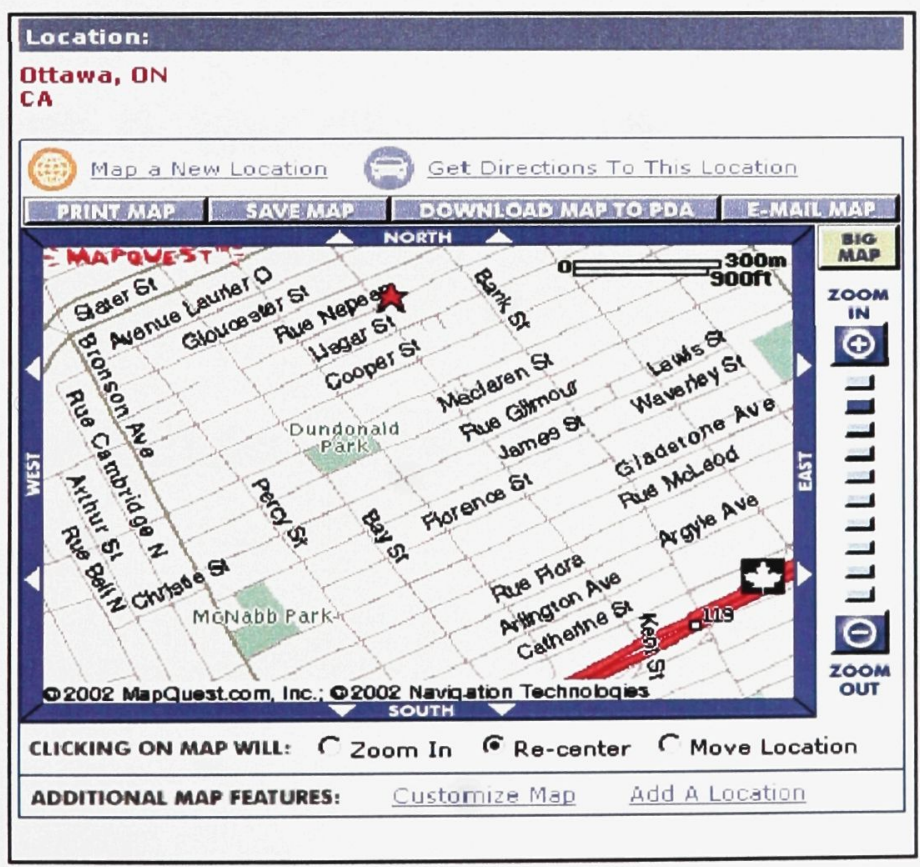


As a typical commercial Webmapping site, MapQuest also promote their customizable mapping solutions, their interactive mapping products and services, including: the Connect Product Line, Locator, Enterprise Toolkit, NT Server and Cartographic Service for the profit purpose.

\section{Realtor.Com by RealSelect Inc}

URL: http://www.realtor.com/

Realtor.com is an interactive real estate website developed by RealSelect Inc. to help people purchasing houses. Realtor.com has all the map data of Canada and the United States for performing a spatial query to find all housing resources within a certain area. Figure 3.3.9, Figure3.3.10, Figure 3.3.11 and Figure 3.3.12 demonstrated a sample of housing search within Ottawa area. House property searches can be narrowed to a list of suitable houses by filling a detailed checklist of price range, the number of bedrooms, bathrooms etc. information. After just a few clicks, the houses meeting your criteria will pop up on the screen. By trying different combinations of the query criteria or query different areas of a city, it will be easy to find several potential locations to visit.

Realtor.com has been developed based on ESRI's MapObject IMS and the data source is from GDT Company. 


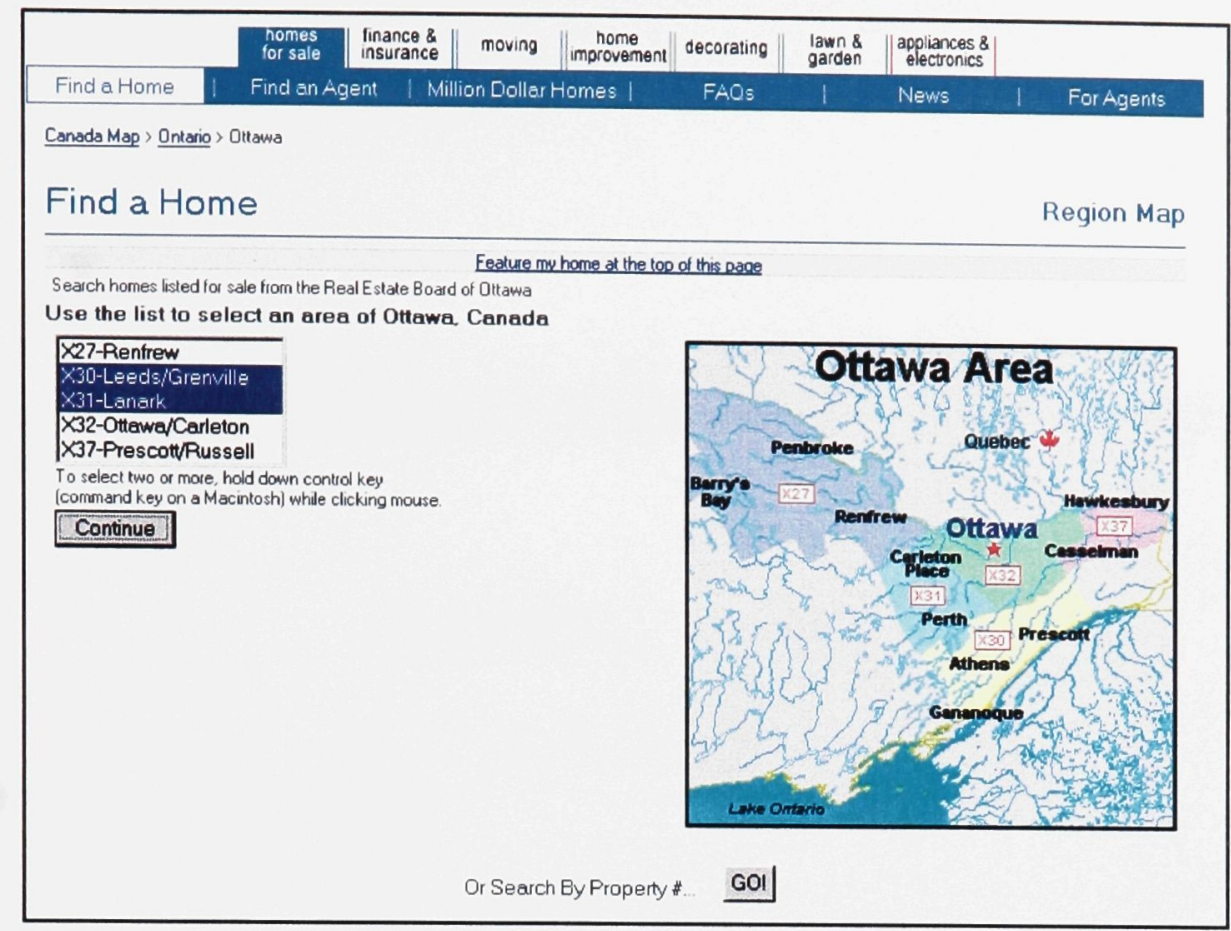

Figure 3.3.9 Housing Property Query: Select Ottawa Area

\begin{tabular}{|c|c|c|c|c|c|c|c|c|c|}
\hline \multicolumn{10}{|c|}{ (s) homestore } \\
\hline Find a Home & I Find an & Igent & \multicolumn{3}{|c|}{ | Million Dollar Homes | } & \multicolumn{2}{|l|}{ FAQs } & News & For Agents \\
\hline \multicolumn{10}{|c|}{ Canada Map $>$ > > Sub-Region > Property Type } \\
\hline \multicolumn{4}{|c|}{ Find a Home } & & & & & \multicolumn{2}{|c|}{ Select Property Type } \\
\hline \multicolumn{10}{|c|}{ Select a Property Type to search within the selected areas. } \\
\hline & & & & \multicolumn{3}{|c|}{$\begin{array}{l}\text { - Single Family Home } \\
\text { r Townhome or Condo } \\
r \text { Multi-Family Home } \\
r \text { Land }\end{array}$} & & & \\
\hline & & & & \multicolumn{2}{|r|}{ Cont inue } & & & & \\
\hline Home Page & Homes For Sale & Finance & Moving & & ne Improveme & Deco & rating & Lawn \& Garden & Appliances \& Electronics \\
\hline
\end{tabular}

Figure 3.3.10 Housing Property Query: Select A Property Type 


\section{숭 homestore"}

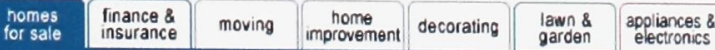

Find a Home | Find an Agent | Million Dollar Homes | FAQs | News Igents

Canada Map > > > Sub-Region > Property Type > Criteria

Find a Home

$\begin{array}{cl}31 \text { Listings } & \text { See them all } \\ \text { match your search criteria } & \text { Narrow the list! }\end{array}$

GeneralSearch Criteria

Approximate Price Range:

$\$ 0$ to no maximum

Minimum Number of Bedrooms: any

Minimum Number of Baths: any

Display Listings in Groups of: 6

View Listings:

Faster: with photos

Search!

- Or, add more criteria! -

Detailed Search Criteria (optional)

Listings with these features will be presented first

\section{Figure 3.3.11 Housing Property Query: Fill in Search Criteria}

\section{Canada Map > Change Criterie \\ SearchResults}

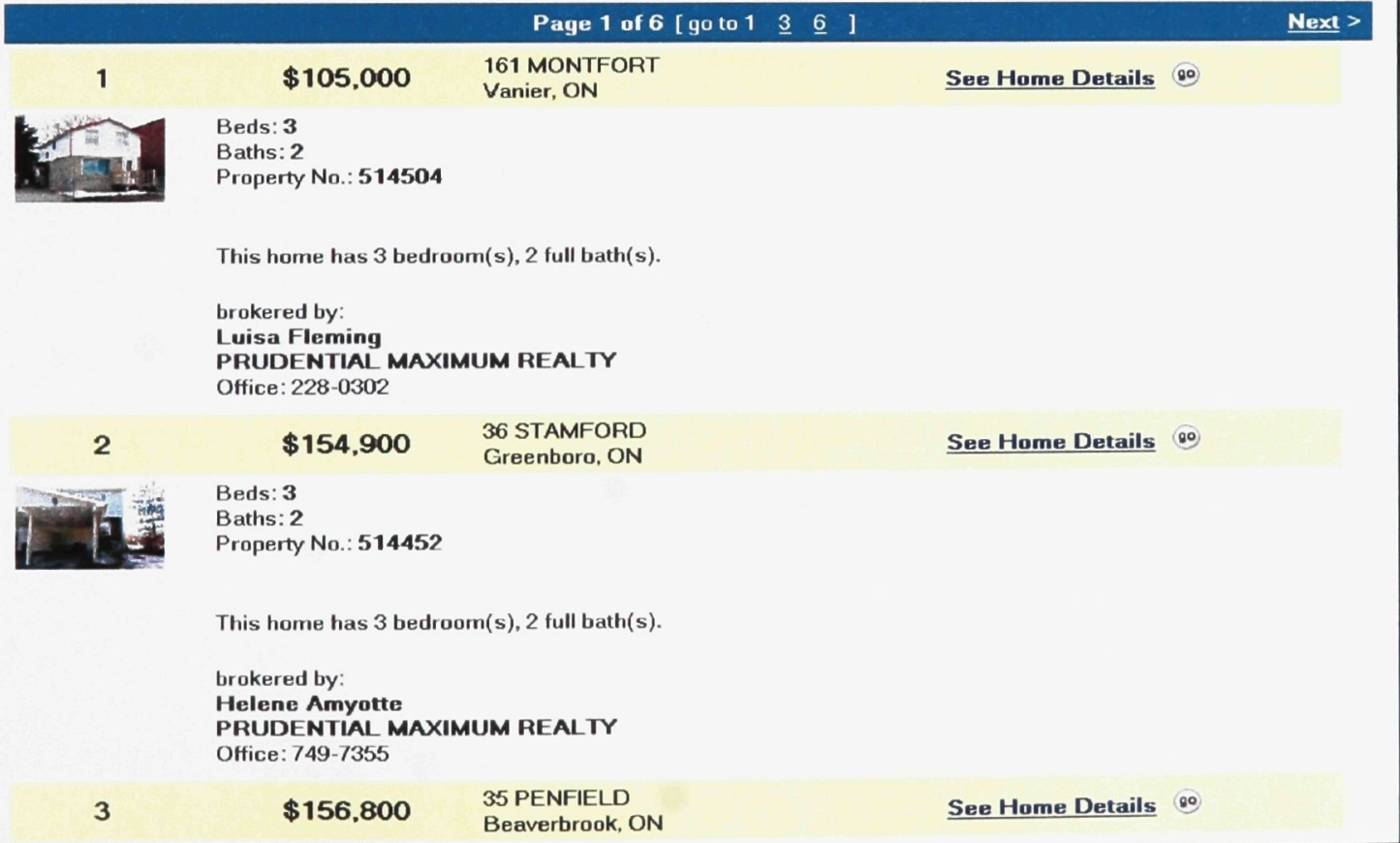

Figure 3.3.12 Housing Property Query: Result Display 


\subsection{Conclusion}

Like everything on the Internet, Web mapping tools and web mapping service are constantly changing. The capabilities of many Web mapping solutions today are very different from those of yesterday, and will be very different from those of tomorrow. The investigating of Internet mapping solutions through the Web is just scratching the surface of the possibilities in this realm. It is difficult to determine or obtain detailed descriptions of the structural design and the site configuration approach utilised on most application from the Internet. Therefore, a further exploration using a case study is important to help achieve a better understanding of the best approach to online geospatial application implementation. 


\section{CHAPTER 4. A CASE STUDY - CONSTRUCTING ONLINE OTTAWA HOUSING ATLAS}

\subsection{Introduction}

Housing is more than shelter since it is where individuals can feel secure, comfortable, and part of the community. It is the focus of our daily environment. Most people spend more on housing than on any other single item they purchase. A house also becomes one means of achieving basic financial security and stability.

All levels of government: federal, provincial, regional and municipal, take an active interest in housing. For example, the federal government intervenes through the tax systems and takes Canada Mortgage and Housing Corporation (CMHC) as the primary mechanism for federal housing action. The province also sets a broad framework for housing policies and rent control. The regional and local municipalities formulate important housing policies and plans to accommodate the changing needs of the population. Therefore, easy and efficient access to housing information is critical for both government and the public.

Up-to-date Webmapping technology adds additional value to the statistic information on housing. It enables housing information to be more accessible through the Internet in a creative way. Thus, presenting housing information through an intuitive visualization approach will provide efficient support for both regional housing policy makers and the 
public to access housing related information.

In this chapter, city of Ottawa housing information has been chosen as an example to construct a Web based geospatial information distribution prototype, 'Online Ottawa Housing Atlas' (OOHA). The fundamental site configuration strategy and process of the Web based spatial information network architecture will be explored through this case study. The valuable roles that Webmapping is playing in society will be demonstrated as well. Thus, the approach of serving society by interactive web based maps will be examined through the implementation of OOHA application and the publication of Ottawa housing information via Carleton University's HTTP server. The advantages and limitations of the online Ottawa housing visualization approach will be discussed.

\subsubsection{Background}

Spatial statistical data can be a useful tool in supporting the strategic and operational needs of various organizations. In the housing industry, housing statistical data are often used for monitoring housing conditions in changing social and economic circumstances. Statistic data in housing provides up-to-date information to inform and assist decisionmakers, planners and housing policy development by industry and government.

\subsubsection{Objectives of the Case Study}

The objectives of this case study are twofold. First, this study explores the approach of using the Internet tool to distribute geospatial information to a wider audience. Second, this case study is to further extend the web-based access to Ottawa housing statistical data 
in a visualization manner and to present the spatio-temporal distribution pattern of housing conditions within Ottawa area in 1991 and 1996. In order to implement this, an existing public domain web mapping software toolkit, MapServer, from the University of Minnesota, will be used to develop the OOHA prototype and publish a housing atlas through Carleton University Website.

This case study seeks to address the housing requirements and housing status in the city of Ottawa in 1991 and 1996 by revealing the spatio-temporal distribution of household characteristics and housing socio-economic contexts. The specific objectives are:

1. Develop a feasible distribution network architecture, Online Ottawa Housing Atlas Prototype, to improve data access to the housing statistic information and broaden web mapping community.

2. Make housing statistical information accessible to the public in a flexible and intuitive manner (maps). Also, promote the visualized socio-economic representation via the Internet.

3. Reveal the spatio-temporal variation of housing needs in the Ottawa region from 1991 to 1996 .

\subsection{Data and Study Area}

\subsubsection{Study Area}


The study area is the City of Ottawa which is comprised of the following municipalities:

Ottawa, West Carleton, Kanata, Nepean, Goulbourn, Rideau, Osgoode, Gloucester, Vanier, Rockliffe Park and Cumberland.

\subsubsection{Data and Variables}

The housing statistics, derived from Statistics Canada and CMHC, provide a snapshot of housing conditions in the Ottawa region. These are especially valuable for small scale studies and overall trends.

\section{Data Source}

OOHA is designed to present and examine the spatial and temporal distribution of Ottawa housing condition among 11 municipalities of the City of Ottawa by the key variables of household characteristics, housing stock and market characteristics. The Atlas maps the existing demographic, economic and housing market data to encourage a visualization approach of housing needs analysis within Ottawa.

The data used are based on the following micro data files from Statistics Canada and CMHC: Profile Series Census 1991 (Statistics Canada), Profile Series Census 1996 (Statistics Canada), 1991 Annual Housing Review (CMHC), 1996 Housing Market Outlook (CMHC). The other geographic map layers, include: Community Centers, City Roads, City Rivers, Ottawa Regional Map, Land Use Map are from the City of Ottawa Thematic Map (MTM, Zone9, NAD83). They are integrated to give basic spatial context to the housing demographic and economic features. 
It is important to note that the data used for presentation and analyses are based on various authoritative sources (Statistics Canada, the City of Ottawa and CMHC) believed to be reliable. The census profiles contains thousands of variables from which only those key variables of household characteristics, house stock and market characteristics have been compiled and used to indicate the housing conditions and housing needs in 1991 and 1996. The spatial unit of the census variables is Census Subdivision (CSD) geographic level which coincide with each municipality boundary.

\section{Variables and Definition}

All the variables used are divided into the following three categories:

- Household Characteristics,

Key variables were:

1.) Household Type: refers to family and non-family household types

2.) Household Income: refers to the average household income

3.) Age of Household $\mathrm{Head}^{2}$ and Population Age Group

Age Groups are divided into: Age 0-24, Age 25-34, Age 35-44, Age 45-54, Age 55-64 and Age 65+.

\footnotetext{
${ }^{2}$ Note: as "Age of Household Head" is not available in the published census data, the variable of "Population Age Group" has been used as an alternative to reflect the geographical distribution of housing demographic features of different age group within city of Ottawa.
} 
- General Demographic and Economic Factors

1.) Demographic Factors in 1991 and 1996

Key variables used:

- Population

- Population Age Group

- Total Number of Household

2.) Economic Factors in 1991 and 1996

Key variables used:

- Average Household Income

- Unemployment Rate (refers to the population of age 25 or over)

- Housing Stock and Market Characteristics

1.) Housing Stock Characteristics in 1991 and 1996

Key variable used:

- Occupied Private Dwellings by Structural Type

Includes: Single Detached House, Semi-Detached House, Row House, Apartment Detached Duplex, Apartment Building 5 Stories or More, Apartment Building Less Than 5 Stories, Other Single Detached House and Movable Dwelling

2.) Housing Market Characteristics in 1991 and 1996

Key variables used: 
- $\quad$ Average Dwelling Price

- Average Gross Monthly Rent

- Gross Rent Spending 30\% or More of Household Income

- Average Owner's Major Monthly Payments

- Owner's Payments Spending 30\% or More of Household Income

- $\quad$ Total Housing Starts

\section{DEFINITION OF VARIABLES}

The variables used in this case study have been defined below according to the "1991 CENSUS DICTIONARY" (Statistics Canada, 1991) and "1996 CENSUS DICTIONARY" (Statistic Canada, 1996).

- Household Type

"Refers to the basic division of private households into family and non-family households."

\section{- Family Household}

"Refers to a household that contains at least one census family, that is a married couple with or without never-married sons or daughters, or a couple living common-law with or without never-married sons or daughters, or a lone parent living with one or more never-married sons or daughters (lone-parent family)." 


\section{- Non-family Household}

"Refers to either one person living alone in a private dwelling or to a group of two or more people who share a private dwelling, but who do not constitute a census family."

\section{- Census Family}

"Refers to a now-married couple (with or without never-married sons and/or daughters of either or both spouses), a couple living common-law (with or without never-married sons and/or daughters of either or both partners) or a lone parent of any marital status, with at least one never-married son or daughter living in the same dwelling."

- Average Income of Households

"Average household income refers to the weighted mean total income of households."

\section{- Total Income of Household}

"The total income of a household is the sum of the total incomes of all members of that household."

- Age

"Refers to the age at last birthday (as of the census reference date, May 14, 
1996/1991). This variable is derived from the date of birth."

\section{- Population Age Group}

"Population age group is defined as the total number of population within a certain age range in a given area. "

\section{- Unemployment Rate}

"Refers to the unemployed labour force expressed as a percentage of the total labour force in the week (Sunday to Saturday) prior to Census Day. Data are available for persons 25 years of age and over, excluding institutional residents."

\section{- Unemployed}

"Refers to persons 25 years of age and over, excluding institutional residents, who, during the week (Sunday to Saturday) prior to Census Day, were without paid work and were available for work and either:

(a) had actively looked for work in the past four weeks; or

(b) were on temporary lay-off and expected to return to their job; or

(c) had definite arrangements to start a new job in four weeks or less."

\section{- Dwelling}

"Refers to a set of living quarters in which a person or a group of persons reside or could reside." 


\section{- Occupied private dwelling}

"Refers to a private dwelling in which a person or a group of persons are permanently residing. Also included are private dwellings whose usual residents are temporarily absent on Census Day. Unless otherwise specified, all data in housing reports are for occupied private dwellings rather than unoccupied private dwellings or dwellings occupied solely by foreign and/or temporary residents."

\section{- Structural type of dwelling}

"Refers to the structural characteristics and/or dwelling configuration, that is, whether the dwelling is a single-detached house, an apartment in a high-rise building, a row house, a mobile home, etc."

- Movable dwelling

"Includes mobile homes and other movable dwellings such as houseboats and railroad cars."

- Average value of dwelling

"Refers to the dollar amount expected by the owner if the dwelling were to be sold."

- Tenure

"Refers to whether some member of the household owns or rents the dwelling, or 
whether the dwelling is Band housing (on an Indian reserve or settlement)."

- Average gross rent

"Refers to the total average monthly payments paid by tenant households to secure shelter".

- Average owner's major monthly payments

"Refers to the total average monthly payments made by owner households to secure shelter."

- Owner's Major Payments or Gross Rent as a Percentage of Household Income

"Refers to the proportion of average monthly total household income which is spent on owner's major payments (in the case of owner-occupied dwellings) or on gross rent (in the case of tenant-occupied dwellings). "

- Gross rent spending $30 \%$ or more of household income

"Refers to the total number of tenant households that spending $30 \%$ or more of household income on shelter costs"

- Owner's payments spending $30 \%$ or more of household income

"Refers to the total number of owner household that spending $30 \%$ or more of household income on shelter costs" 


\subsection{Methodology}

\subsubsection{Introduction to the System}

The OOHA is designed as a distributed spatial data service application that allows people to browse housing data in relation to geographic and statistical variables. The main goal is to provide access to the Ottawa housing information through the Website of Carleton University. The basic system environment and specifications are as follows:

- Application Name: Online Ottawa Housing Atlas (OOHA)

- Web Server: Apache/1.3.22 Server at http://www.carleton.ca, Port 80

- Operation System Environment: Server side-Unix, Client side - NT

- Screen Resolution: 1024 x 768 or above

- Development Platform:

- MapServer: MapServer by University of Minnesota, Version 3.5, Unix

- Web Browser: Internet Explore Version 5.5 or above

- Development Tools:

HTML, MapScript from MapServer V3.5, Minnesota Univ. and JavaScript

- Spatial Data Processing System: Arc/View GIS Desktop,V3.2, ESRI

- Data Format:

- “.html” for application interface template file

- “.map" ( mapscript file) for map display administration tool

- " "shp" for map data 
- “.png”, “.jpg”, “.gif” for keymap, icon and interface button

- Web Site

- URL: http://www.carleton.ca/ lyu/Happy Housing.HTM

\subsubsection{System Design and System Development}

\subsubsection{System Design}

\section{- Client/Server Solution Architecture}

As introduced in Chapter2, most of the "open source" and "off-the-shelf" WebGIS often use a server side interactive site configuration approach to construct Webmapping application. The request and process to the geospatial data are "served" by a host from the server side. OOHA prototype has used this typical site configuration model. A designed strategy model of OOHA has been designed and illustrated in figure 4.3.1.

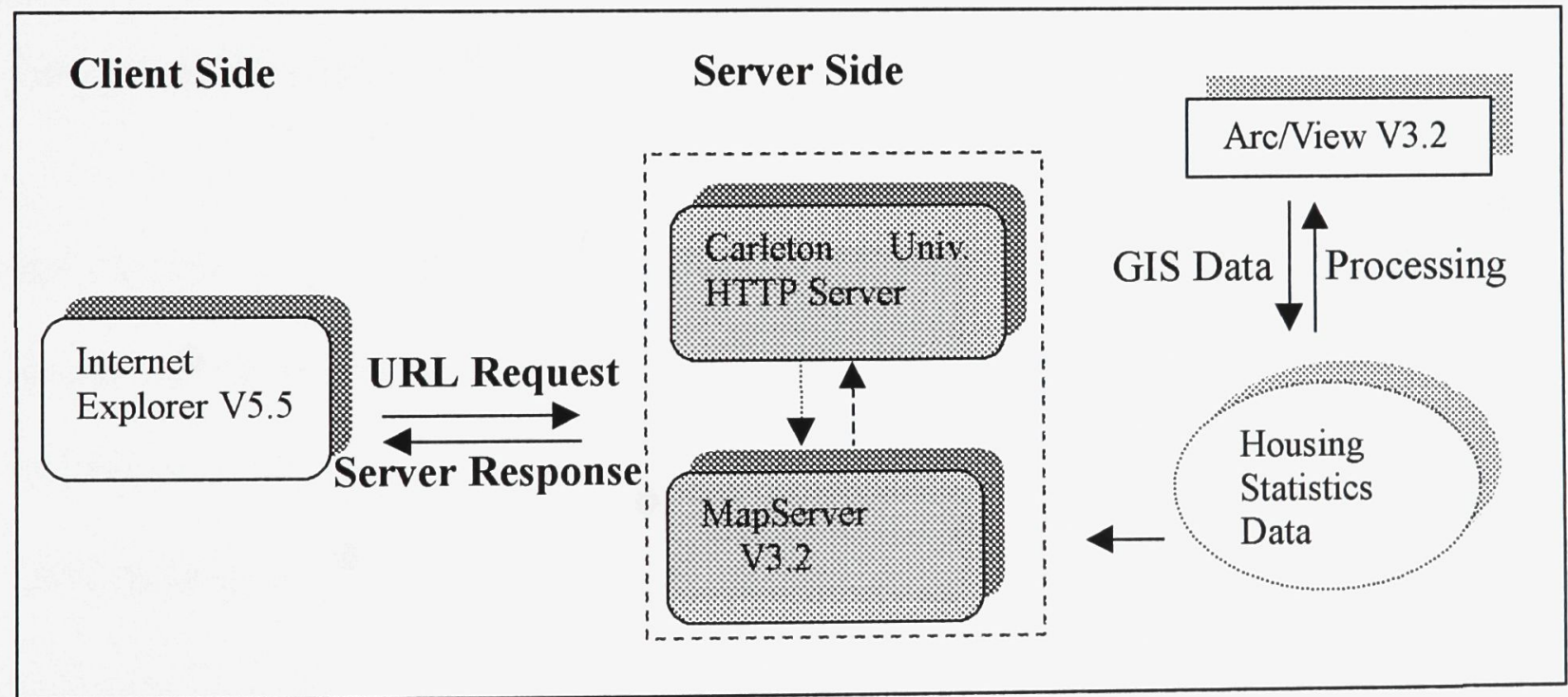

\subsubsection{Online Ottawa Housing Atlas Application Server Side Strategy Model}


- The general communication flow of the OOHA model is the following:

1. A request from Internet Explorer (IE) is sent to the Carleton University server across the Web.

2. The university server processes the requests, renders the housing data handling request to the MapServer, which resides on the same site as the university HTTP server.

3. The MapServer processes the spatial data and sends result back to the HTTP server.

4. The response is then sent back to the user by HTTP server and displayed via IE.

\subsubsection{System Development}

\section{- MAPSERVER Platform}

In terms of the underlying technology of this case study, the most critical is the choice of the developing platform. In this study, MapServer Version 3.5, an open source approach has been utilized as the primary development base.

As described in chapter 3, MapServer (http://mapserver.gis.umn.edu) is a free downloadable Webmapping engine for building spatially enabled Internet applications. It is maintained by the TerraSIP project, a NASA sponsored project between the University of Minnesota and a consortium of land management interests.

Moreover, MapServer is a CGI server-side application. It uses the Common Gateway Interface (CGI) standard to interact with the HTTP server. Since this standard has been 
around since the early days of the Web, MapServer can run with any HTTP server and on operating systems including, but not limited to: Windows NT/95/98, Linux, Sun Solaris, SunOS 4.x, Irix and FreeBSD. Applications built with MapServer produce output compliant with the World Wide Web Consortium (W3C) HTML 3.2 specifications.

As pointed out in the former chapter, MapServer is not designed to be a full-featured GIS system, nor does it aspire to be. However, it does provide basic functionality to support a wide variety of Webmapping applications. It appears to be a robust and effective customization tool, which is particularly suitable for a Web application developer, but not direct users. Considering the capabilities of MapServer, the academic research purpose, the financial and time limitations of this study, this open source approach is ideal for building-up the OOHA application.

\section{- Application Development}

Publishing maps requires putting them through several different software packages (Wim Feringa, 2000, P177 Web Cartography). In order to facilitate the linking of the servers and to make data interactively presentable through the Web, additional custom tools need to be developed to integrate the different software packages and to publish maps:

1. A map display administration tool (".map" file) has been developed, allowing the data to be made accessible, and to be set-up locally or remotely through an easy-to-use Web interface. It will be through this interface that such things as line styles, 
symbology, colours, classifications and other data representation criteria will be set. MapScript, defined by MapServer software, is used to develop this tool.

2. A selection of mapping tools, such as keymaps, pan for navigation, zoom in and out, have been developed to be used as "plug and play" for customizing the OOHA Web mapping interface. Also, such things as selected layers and interactive functionalities have been set up. This set of mapping tools can be used to develop the other different interfaces of spatial data viewer to meet the specific needs of the end users.

Another point for designing this application is the Internet protocol. In order to better support the Web based distribution applications, Java/JavaScript and HTML/DHTML, together with a commonly agreed domain-specific Internet protocol, formed a compatible basis for designing a distributed interoperable Web mapping viewer based on MapServer and a standard Web browser. Thus, the OOHA viewer interface has been developed based on Internet Explorer using HTML and JavaScript.

\subsubsection{Implementation}

One of the primary goals for establishing this case is to develop a public domain distributed spatial statistics data service. By adhering to this principle, the OOHA Web mapping application encourages information sharing, identification functions and easy access to housing related socio-economic data.

\subsubsection{Issue for OOHA Site Design}


An Internet site is more than just a couple of pages with contents stitched together with some hyperlinks. The basis is the information that has to be transferred. Thus, a site designer's main concern is how the information will reach the user in the easiest and most efficient way. As suggested in Chapter 2, five primary considerations need to be addressed: Site Configuration, Site Format, WWW Interface, WWW Data Type and Content Interface.

\section{$\underline{\text { Site Configuration }}$}

This determines the primary interactive process location with respect to the basic client/server model introduced in the former section. As indicated in Figure 4.3.1, OOHA is a server side interactive Webmapping application. All the interactive processes, such as Spatial Query, Zoom In, Zoom Out, Recenter, proceeded within MapServer which resides at the server side of the school Internet. Then, the Web Server sends back to the client side to be browsed.

\section{$\underline{\text { Site Formart }}$}

here are three types of site format: Single Page, Multiple Pages and Frames. This case has adopted the most straightforward single page site format. The interface of OOHA has all its contents and interactive functions on one page which makes the application clearer and more user friendly. Figure 4.3.2 shows the OOHA viewer interface. 


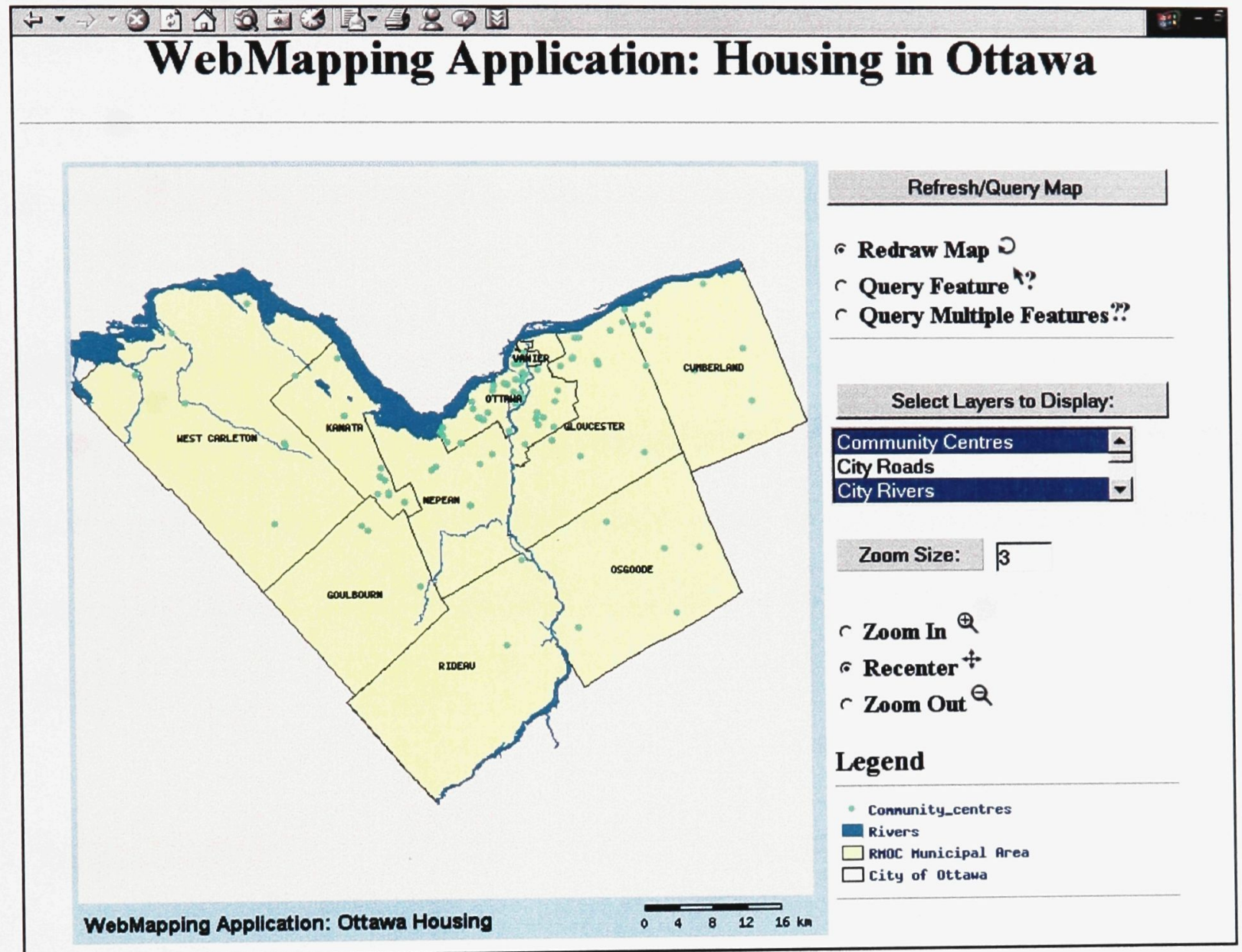

Figure 4.3.2 the Online Ottawa Housing Application (OOHA) Interface 


\section{WWW Interface}

As Microsoft Internet Explorer supports the standard Hypertext Transfer Protocol communication, HTML and JavaScript data format internally, the interface of this application has been built upon the interface of Microsoft Internet Explorer (IE) version 5.5 or above. Additionally, as figure 4.3.2 has illustrated, the OOHA application has its own customized mapviewer interface besides the regular interface of IE.

\section{Web Data Format}

The most popular and fundamental Web design scripts, HTML and JavaScript, have been used in OOHA as the basic Web data format. Javascript can be embedded as the active components in an HTML document to jointly create more intelligent interactive functions.

\section{Content Interface}

Both the content interface design and the Web interface design are significant but very complex. Different user groups and applications have different requirements for the interface design. It is difficult to set up any fixed interface design criteria. However, the interactive map content interfaces can be basically categorized as either generic or customized. For this case, a specific customized map viewer interface based on Microsoft IE has been developed as the OOHA application interface. Furthermore, some interactive operation standards, such as Recenter (Pan), Zoom in, Zoom out buttons have been employed for dynamic communication between the Web browser with the MapServer. However, the user groups in this case have been defined as the general public and 
government policy makers, the specific content interface of the OOHA has been designed to be a simple but typical map viewer interface which contains the basic map display and query tools (Figure 4.3.2 the OOHA Interface). The detail OOHA content interface and its functionality are described in the following section.

\subsubsection{Content Interface and Functionality}

The developed OOHA prototype is a customized Webmapping tool that allows the user to examine housing data in relation to a rich assortment of geographic context.

It provides users with a tool to view and analyze the geographic presentation of housing statistics specific to their interests.

As the interface of OOHA application demonstrates, the interface is broken into two main regions on the screen.

The MapView occupies most of the left part of the screen. It is used as the primary visual focus of OOHA. The MapView allows the user to specify maps from the list of map layers to display. Each map layer represents a category of data, such as housing price or household income over a topographical area. Most of the functions of the toolbar are performed upon the data represented in the Mapview.

- The Toolbar is situated on the right-hand side of the screen, and includes:

- Redraw Map:

- $\quad$ Single Layer Query

- $\quad$ Multiple Layer Query 
- $\quad$ Turn On/Off Layers

- Zoom In/Zoom Out

- Recenter

- Zoom Size

Legend, ScaleBar and Keymap also reside at the bottom-right of the OOHA interface.

\subsubsection{Map Edit}

Housing statistics provide the housing condition information and the housing marketing status on an ongoing basis. Based on the available census data and housing statistics, the following variables have been compiled and put online to illustrate Ottawa local housing conditions in 1991 and 1996. This provides the information on how conditions changed between 1991 and 1996. The results can serve to inform and assist housing decisionmakers in industry, all levels of government, the private sector and the general public.

\section{- Variables of Housing Conditions}

- Housing Stock and Market Characteristics Maps:

Layer Name: Housing_Stock_Market91

$$
\text { Housing_Stock_Market96 }
$$

Both layers are classified by average housing price in 1991 and 1996. They provide the housing stock and market information such as different types of dwelling stock, housing starts and average dwelling price. 
- Household Change Map From 1991 to 1996:

$$
\begin{aligned}
& \text { Layer Name: Household_Change } 91 \_96 \\
& \text { These map layers are classified by the number of } \\
& \text { household changes and provide the information including } \\
& \text { population change, household change, the change of } \\
& \text { household income, dwelling price change and } \\
& \text { unemployment change, etc. }
\end{aligned}
$$

- Housing Economic Factor Maps:

Layer Name: Housing Economic Factors91

Housing Economic Factors96

- Housing Demographic Factor Map:

Layer Name: Housing_Demographic Change91_96

- Household Characteristics Maps:

Layer Name: Household Characteristics91

$$
\text { Household Characteristics96 }
$$

The other compiled map layers: Community Centers, City Roads, City Rivers, Ottawa Regional Map, City of Ottawa, are added to give additional spatial context to the housing related demographic and economic variables. 


\section{- Data Extraction and Data Editing}

All data extracted from Census and CMHC are converted into ".dbf" file so that it can be imported into ArcView and joined with the geographic map of the Ottawa region by "table join" tool in ArcView. By joining the statistics with geographics data, the housing statistics are given spatial features that can be presented geographically.

The problem during the data joining procedure is the different data projections in the two sources of data. Ottawa census data extracted from the national census geography data produced by Statistics Canada uses geographic coordinates. However, the Ottawa administrative boundary data to be joined with the census map is generated by the planning division of City of Ottawa. They use the projection of Modified Transverse Mercator (MTM), Zone 9, NAD 83. In order to join both maps, FME - a projection conversion tool, is needed to convert the geographic coordinate of Census map into MTM, NAD83. Since the FME of MAGIC has license problem, an alternative simple solution has been used to implement the data join. The Ottawa housing statistics have been re-extracted in “.dbf" format from orgional census database by Beyond20/20. The ".dbf" file is imported into ArcView. A common name field, "cityname", has been added to the table of census data and the table of Ottawa administrative boundary map. The "table join" tool in ArcView can then easily merge the statistical file with Ottawa administrative boundary data. The spatial distribution of housing statistics can then be displayed and edited by Arc/View after converting the 
merged ".dbf" file into "shapefile.".

\section{- Data Dissemination}

As the open source MapServer system is only a Webmapping tool together with Web Server to publish the spatial data via the Web, it does not have as many map editing functions as regular GIS software. Most of the map editing work in this case has been performed using the ArcView system. Since the ArcView "shapefile" is the default vector data format of MapServer, it easily processes and disseminates spatial data via MapServer.

As was mentioned before, MapServer runs as a CGI engine from the HTTP server. The dissemination of Ottawa housing spatial data requires the following resources:

1. An Apache http server located on the Carleton University server site.

2. A MapServer software, installed in cgi-bin directory of Carleton HTTP server.

3. "Ottawa_init.html" - an initialization file that triggers the first view of the MapServer application.

4. "Ottawa.map" - a Mapfile that controls what MapServer does in OOHA application.

5. "Ottawa.html" - a template file that controls the the OOHA application's user interface in the browser window.

6. A GIS dataset - Ottawa housing spatio-statistics in "shapefile" format. 
OOHA Mapfile, template file, initialization file, and housing atlas datasets are stored in the HTTP server documents directory. Their main functions are described below.

\section{The Initialization File}

"Ottawa_init.html" can be part of the template file -"Ottawa.html", but for simplicity it was developed as a separate file in this case. The initialization file uses a form to send an initial query to the http server that returns a result from the MapServer. MapServer is stateless, and MapServer is started and executed each time a query is received, so this initialization file is just required to pass a variety of (hidden) parameters to the application. The initialization file is a regular html file, so its extension is ".htm" or ".html". Alternatively, a hyperlink to the OOHA can also be constructed. It should pass the basic parameters required by the MapServer CGI application.

\section{The Mapfile}

The OOHA Mapfile -"Ottawa.map", defines the data to be used in an application and the display and the query parameters; This can be considered as the application's configuration file. The Mapfile also includes information about how to draw the map, the legend, and maps resulting from a query. Mapfile has been developed by mapscript and has a ".map" extension

\section{The Template File}

The Template file in OOHA is "Ottawa.html". It controls how the maps and legend 
output from MapServer will appear on an html Webpage; it operates like any other HTML file except that certain fields can be modified by the MapServer CGI. The template file allows the designer to place the map and legend on the page, and determine what ways the user is allowed to interact with the MapServer application (i.e browse, query, zoom, etc.). Mapserver uses the template file and replaces keywords in the template file with information on its current state or the GIS dataset, to produce the html file that is sent to the browser. Since the template file is used to create an html file, it has “.htm" or ".html” extension.

\section{Housing Atlas GIS Dataset}

The Ottawa housing atlas data is stored at HTTP://www.carleton.ca/ lyu/itasca/data in a "shapefile" format.

\subsection{Results and Discussion}

\subsubsection{Result}

By providing visualization housing statistics atlas online, this study examined an intuitive approach for the municipality level housing needs and housing affordability studies in 1991 and 1996. The outcomes of this study have provided an opportunity to review the housing situations of the Ottawa area from 1991 to 1996.

\subsubsection{Regional Housing Context: Socioeconomic and Demographic}

\section{- Socioeconomic Features}


i) Housing Economic Factor Map1991, figure 4.4.1.

Classified by the feature of average household income in 1991

ii) Housing Economic Factor Map1996, figure 4.4.2

Classified by the feature of average household income in 1996

Housing Economic Factor Maps demonstrate the geographic distribution of the average household income within city of Ottawa in 1991 and 1996. They have shown the spatial and temporal variation of the average household income in 1991 and 1996. Both maps provide information on household income, dwelling price, unemployment rate, average monthly rent, average housing owner's payment, affordability, etc.

\section{iii.) Multiple Query}

Query two economic map layers: Figure 4.4.3

Housing Economic Factor91 and Housing Economic_Factors96 


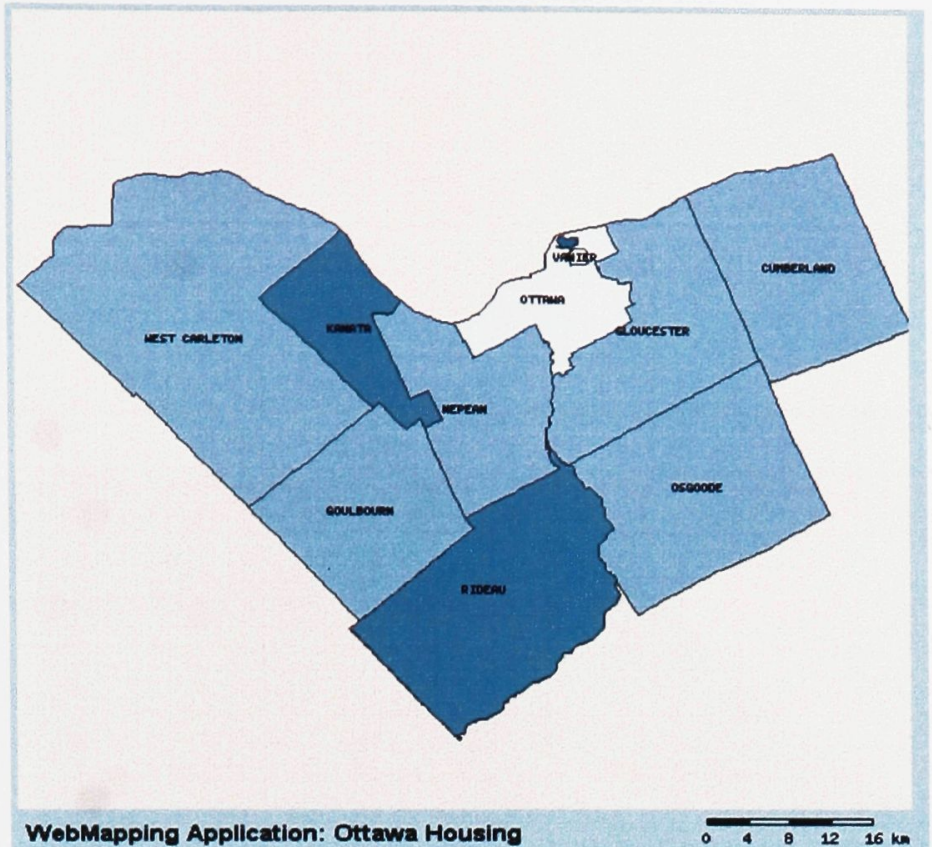

- Redraw Map D

$r$ Query Feature $t ?$

ऽ Query Multiple Features??

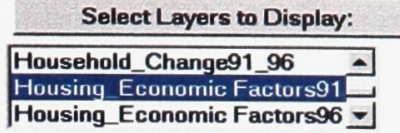

Zoom Size:

r Zoom In ${ }^{\oplus}$

- Recenter +

$r$ Zoom Out $Q$

Legend

$\square$ 91Househld_Incone:<=5e日ee

$\square$ 91Househld_Incone:50001-70e日

91Househld_Incone:78091-108090

91Househld_Incone: over 18989

city of ottawe

Figure 4.4.1 Housing Economic Factors 1991

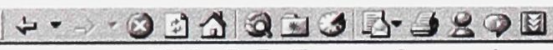 \\ WebMapping Application: Housing in Ottawa \\ (F) -}

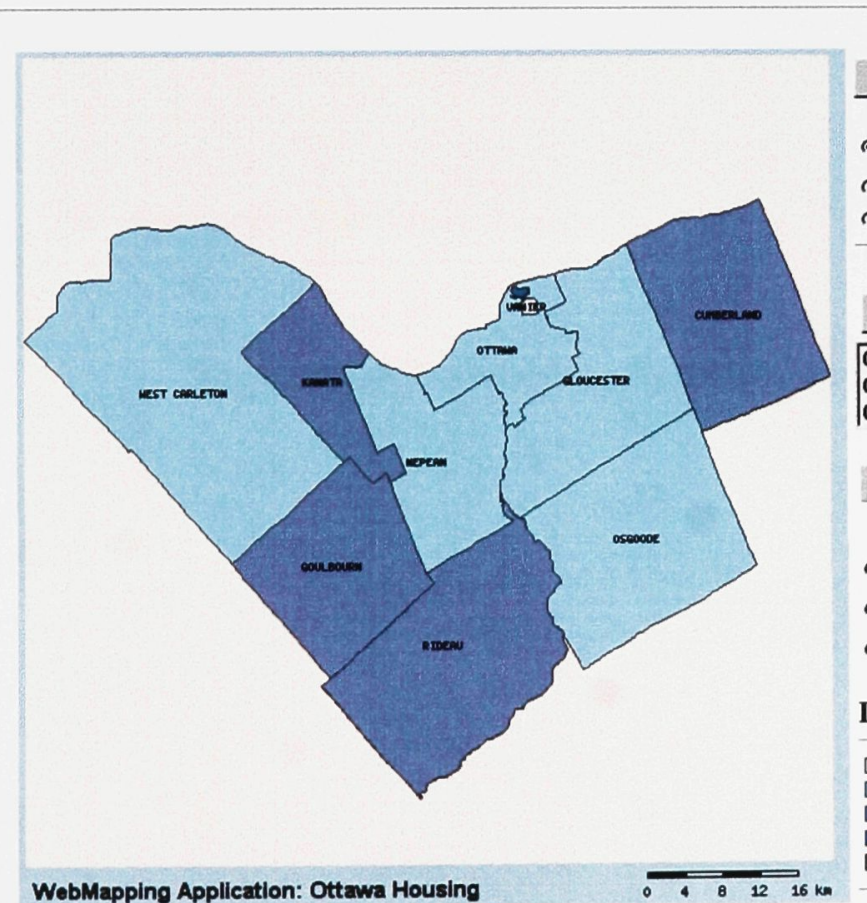

Refresh/Query Map

- Redraw Map D

C Query Feature

ᄃ Query Multiple Features??

Select Layers to Display:

Community Centres 크

City Roads

$=$

Zoom Size: 5

$\sim \operatorname{Zoom}^{\oplus}{ }^{\oplus}$

- Recenter ${ }^{\dagger}$

Z Zoom Out $^{Q}$

\section{Legend}

$\square$ 96Househld_Incone: $<=580 \theta 0$

96househld_Incone:58001-78808

96Househld_Incone:58в81-76888

96hlousehld_Incone: $78081-18080$

WebMapping Application: Ottawa Housing

$481216 \mathrm{k}$

$\square$ city of ottawa

Figure 4.4.2 Housing Economic Factors 1996 


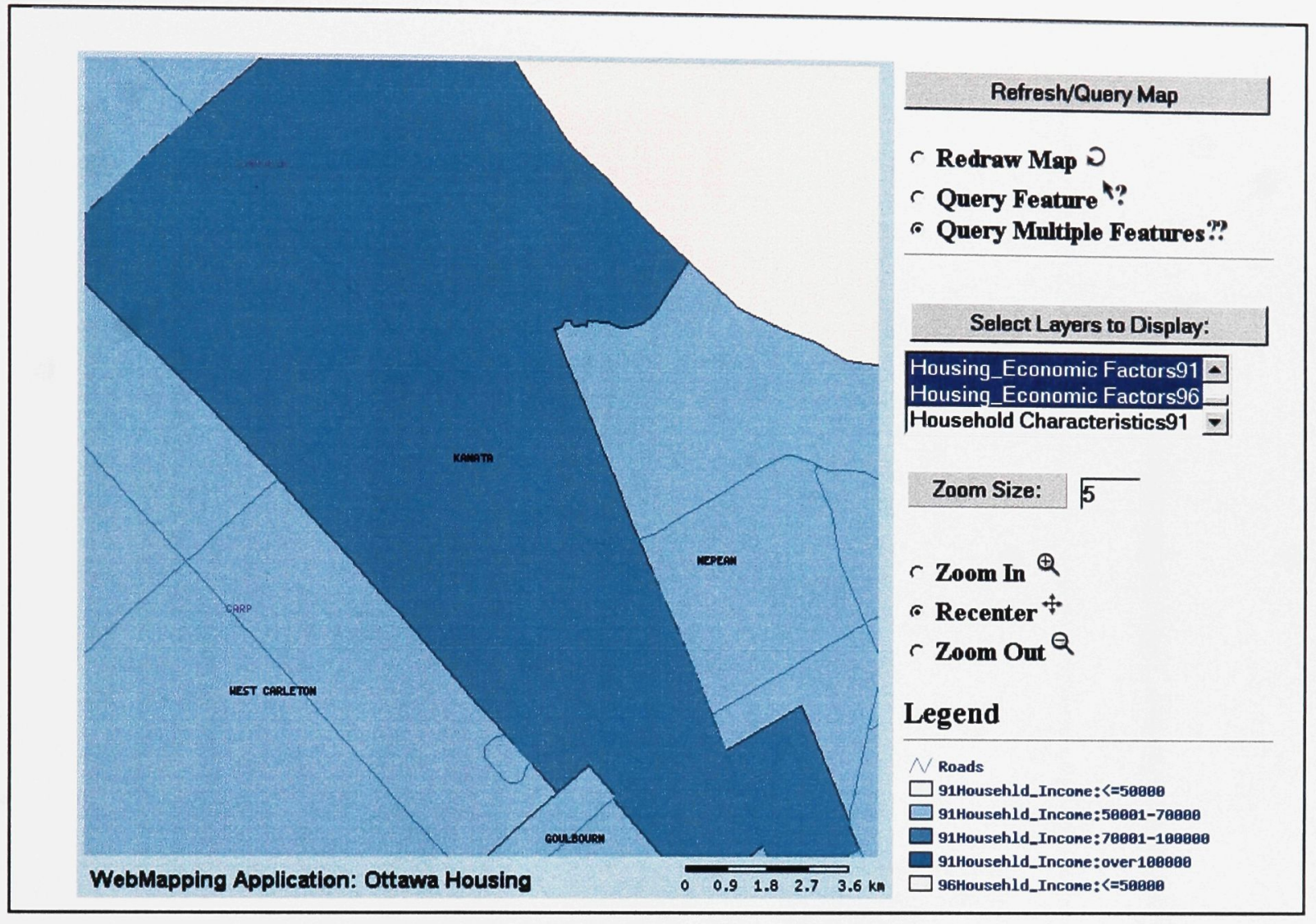

Figure 4.4.3 Housing Economic Feature Query 1991/1996 


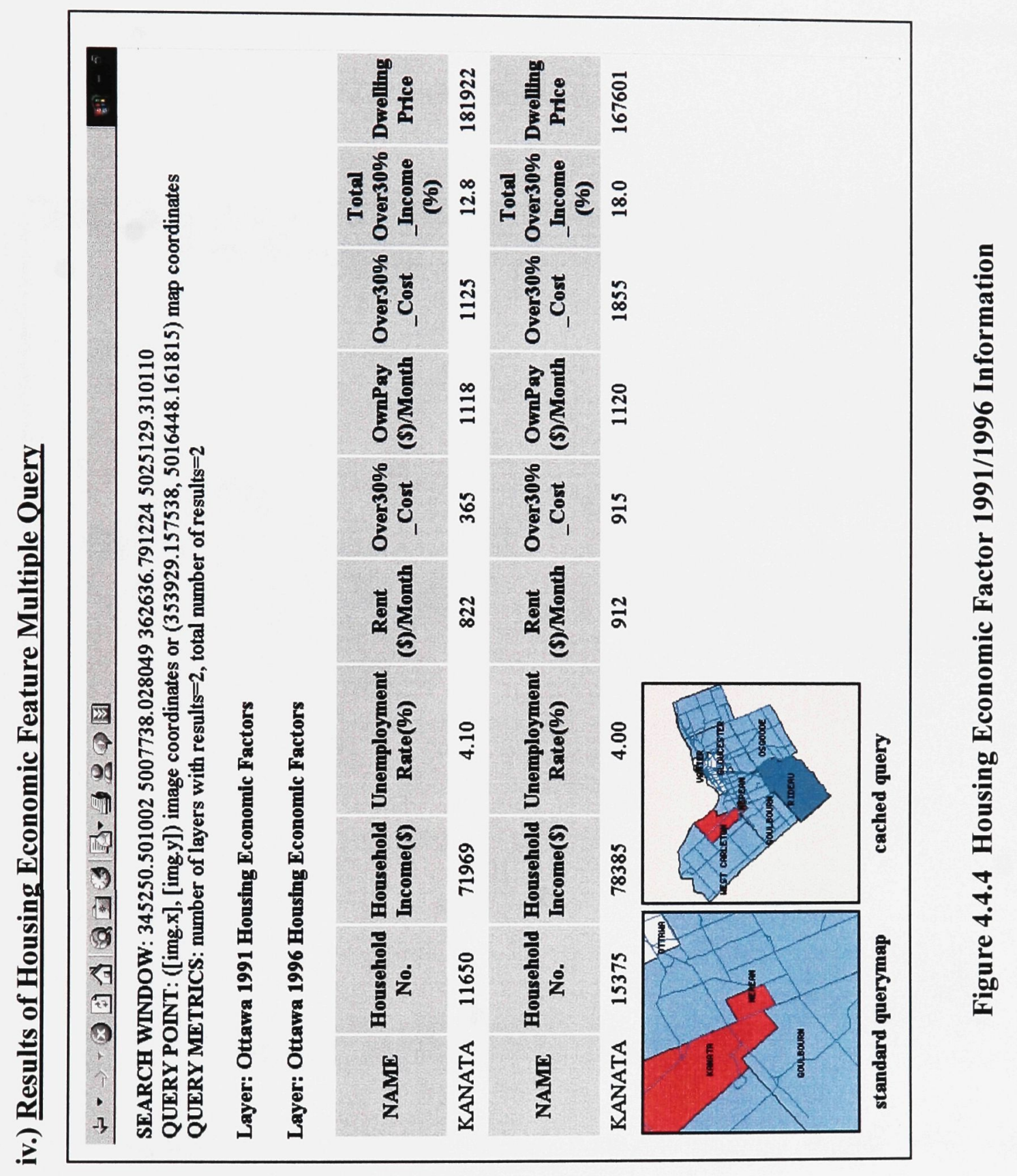


- Demographic

i.) City of Ottawa Housing Demographic Change Map from 1991 to 1996,

Figure 4.4.5

The demographic change maps are classified by the percentage of population change from 1991 to 1996 and indicate the geographic distribution of population change by municipalities. It gives information on population change and the different age groups of population change.

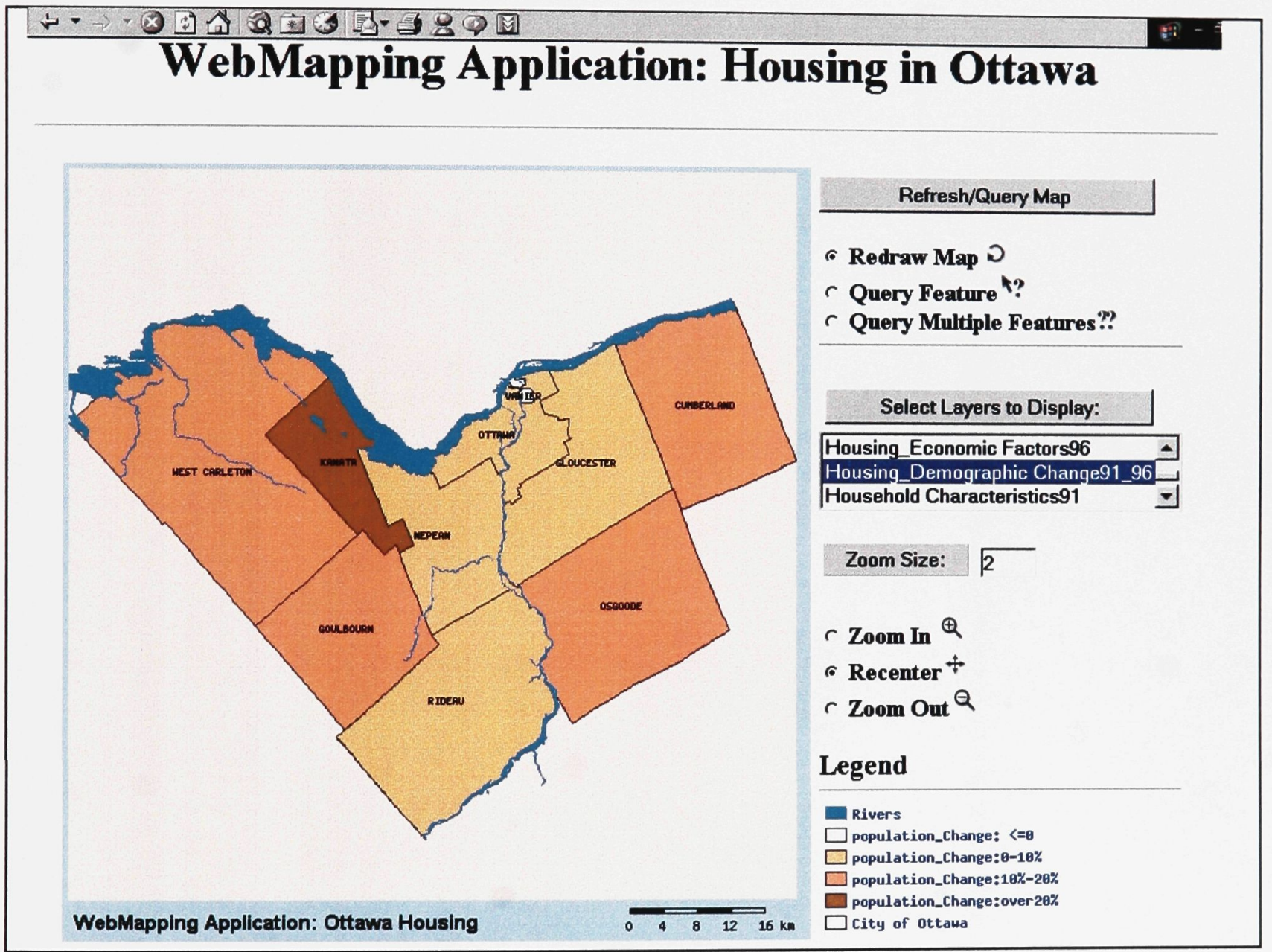

Figure 4.4.5 Housing Demographic Change from 1991 to 1996 


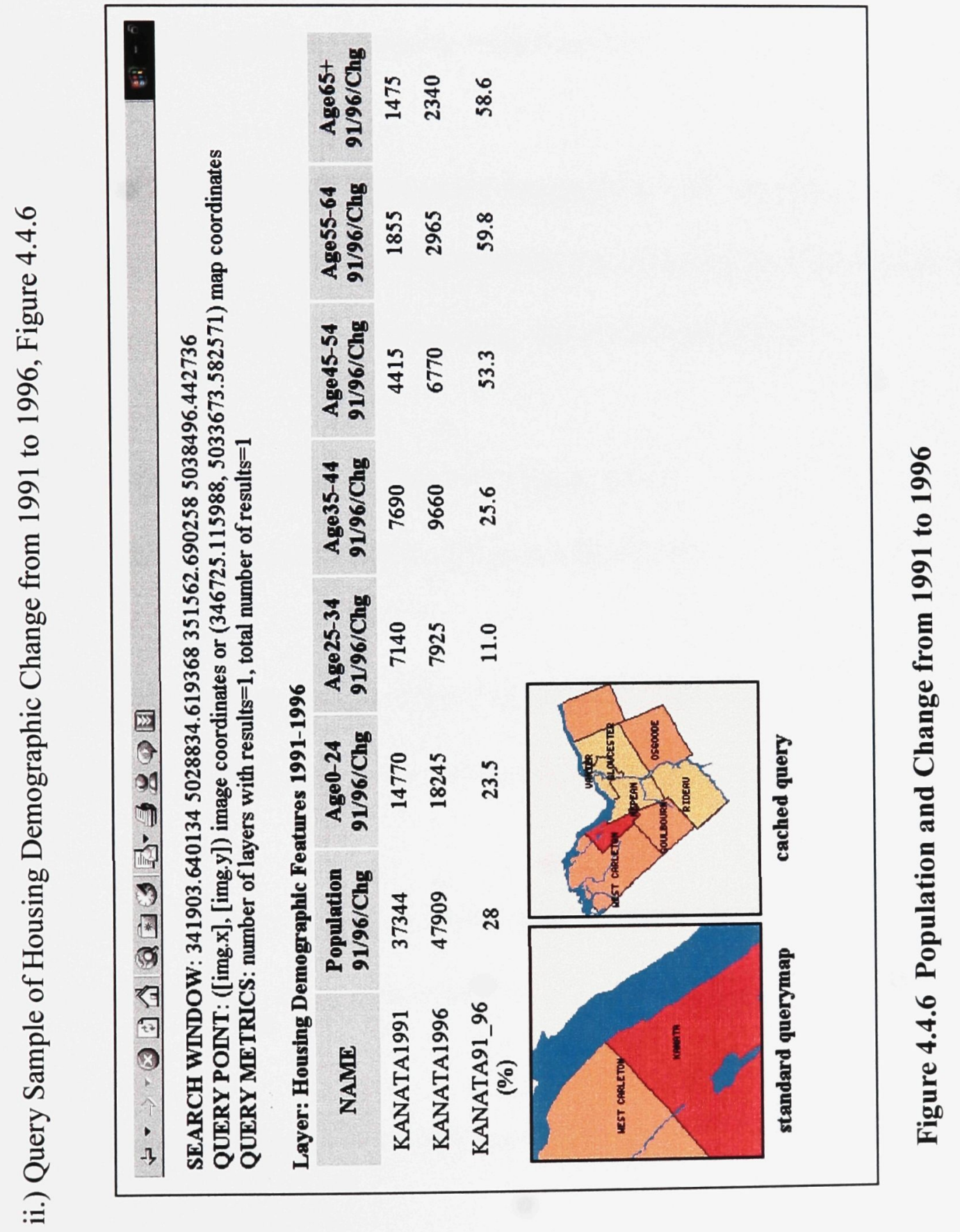




\subsubsection{Housing Needs Indicators}

Household Characteristics mainly referring to the household type, age and income, are the key factor influencing a decision to buy or rent. (Housing in Canada-A statistics Profile, CMHC 1984). The compiled household characteristics map has provided these three important indicators to reflect housing needs status and to offer the fundamental basis for housing plans and housing market analysis.

- Household Characteristics and Change from 1991 to 1996

Household Characteristics Maps present the information of household number, different types of household number, and household income.

i.) Household Characteristics Map1991, figure 4.4.7

Classified by the total number of household in 1991

ii.) Household Characteristics Map1996, figure 4.4.8

Classified by the total number of household in 1996

iii.) Household Characteristics Change Map from 1991 to 1996, figure 4.4.9

Classified by the change in numbers of households from 1991 to 1996

iv.) Household Characteristics Multiple Query Results, Figure 4.4.10

v.) Single Query of the Household Characteristics Change, Figure 4.4.11 


\section{- Analysis Example: Housing Needs and Housing Consumption}

By querying housing economic factor atlas, users are provided with the basic economic context of 1991 and 1996 such as unemployment rate and average household income.

According to the "1991 housing economic factor" map, Rockcliffe Park area shows a very high average household income which is over $\$ 100,000$. Kanata and Rideau area appears to be the $2^{\text {nd }}$ highest household income municipalities which is between $\$ 70,000$ to $\$ 100,000$. The average household income of Ottawa and Vanier appears to be the lowest: both are lower than $\$ 50,000$.

Based on the 1996 housing economic factor map, Rockcliffe Park maintained the highest average household income over other municipalities. The average household income of Goulbourn and Cumberland has increased and become the second highest average household income together with Kanata and Rideau in Ottawa region. The average household income of Ottawa residents has also increased to over $\$ 50,000$ in 1996 . Vanier maintained the same lowest average household income as in 1991 within the City of Ottawa region.

Many households have their specific housing requirements or difficulties finding adequate and affordable housing in the community. The OOHA provides local housing affordability information, such as: tenant monthly rent, owner's monthly payment, the number of tenants or owners who pay over $30 \%$ of the gross income as well as the dwelling price. 
"The tenants or owners who pay over $30 \%$ of gross income" is the commonly accepted measurement of affordability (Regional Housing Statement Update, City of Ottawa, 1991). For example, the query result of the Kanata area housing economic factor map (Figure 4.4.4) indicates that the housing affordability decreased in Kanata city from 1991 to 1996. This is reflected by the amount of household payment over $30 \%$ of gross income. The total number of household paying over $30 \%$ of gross income has increased $5.2 \%$ from $12.8 \%$ in 1991 to $18.0 \%$ in 1996 . However, the decreased housing affordability phenomenon does not match the economic and housing market variation from 1991 to 1996 in Kanata. The average household income shows an increase of $\$ 6416$ from $\$ 71969$ in 1991 to $\$ 78385$ in 1996 . The unemployment rate also decreased from $4.1 \%$ in 1991 to $4.0 \%$ in 1996 . The housing market variable, average dwelling price, demonstrated a certain amount of decrease from $\$ 181,922$ in 1991 to $\$ 167,601$ in 1996. All these economic factors have indicated that the local economy has improved, and as a result, housing affordability should increase.

However, considering the other variables in the query results, it is evident that the decrease in housing affordability (the number of households paying over $30 \%$ income for dwelling cost increased from 1991 to 1996) is due to the increase in numbers of household in this area from 1991 to 1996. Another reason could be that the dwelling price dropped substantially which resulted in a highly competitive housing market. Moreover, the relatively higher household income of Kanata area enabled the households to spend more than $30 \%$ of their gross income for dwelling costs. Another possible reason could be a household type change. As the query result of Household Characteristics Change 
map (Figure 4.4.12 Kanata City Household Characteristics Change) shows, the nonfamily household number has increased $48.9 \%$ from 1991 to 1996 . The non-family household income is usually less than family household income. Therefore, it is very possible for this group of house owners to spend $30 \%$ or more of their gross income on dwelling costs.

The demographic factors of Kanata region, figure 4.4.5 Housing Demographic Change from 1991 to 1996, and Figure 4.4.6 Population and Change from 1991 to 1996, clearly indicate that the Kanata area had the largest population change from 1991 to 1996 . The population change rate in Kanata was $28 \%$ which is far beyond the change rate of the other cities. The query map result, Figure 4.4.6, shows that, the changes among all different population age groups, the change in Age group 35-44 and 45-54 was the greatest. The number of people in Age Group 35-44 increased 1870 from 1991 to 1996. The number of people in Age Group 45 -54 increased 2355 from 1991 to 1996. These two groups are the most capable of spending more on dwelling costs. Considering the household type variation by querying the household characteristics change map, results are listed in the Figure 4.4.12 Kanata City Household Characteristics Change. The query result of the household characteristic change shows that the family type of households increased $29.7 \%$ from 1991 to 1996 . The family type households are usually willing to spend a greater percentage of their income on shelter than non-family individuals. Therefore, all these various socioeconomic factors intervened and caused the specific phenomena of housing requirements and dwelling consumption in the Ottawa region. 


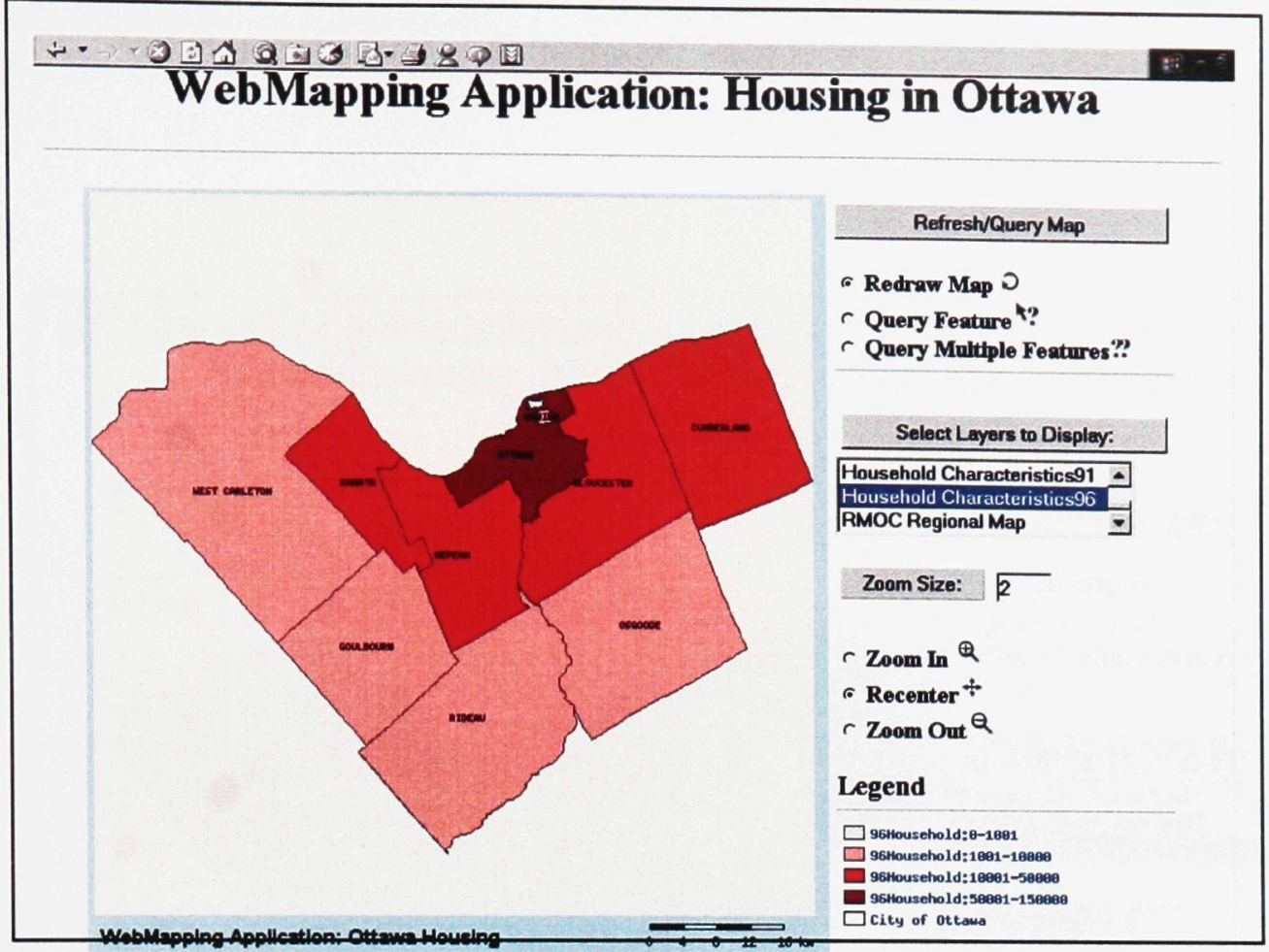

Figure 4.4.7 Household Characteristics Map1991

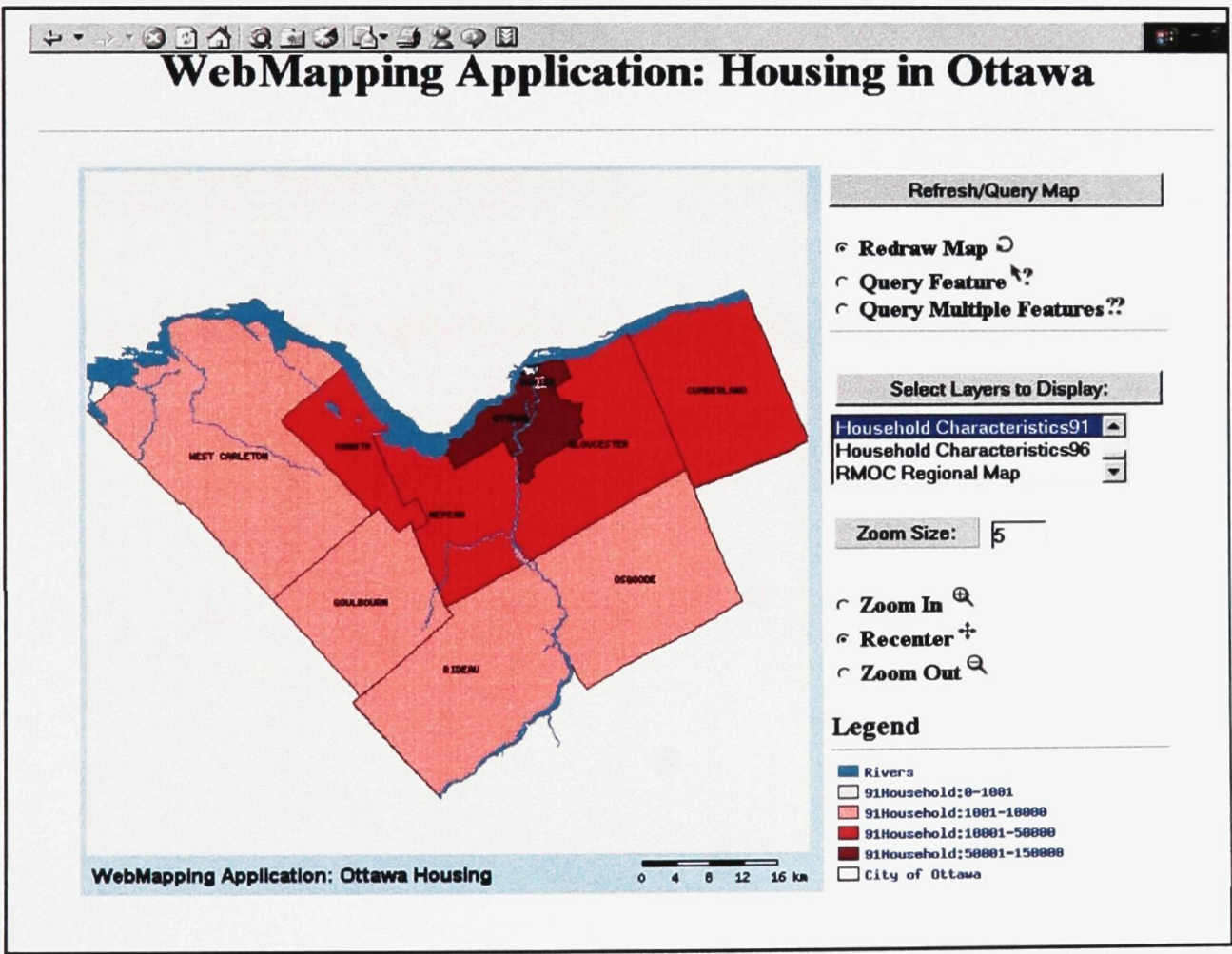

Figure 4.4.8 Household Characteristics Map1996 


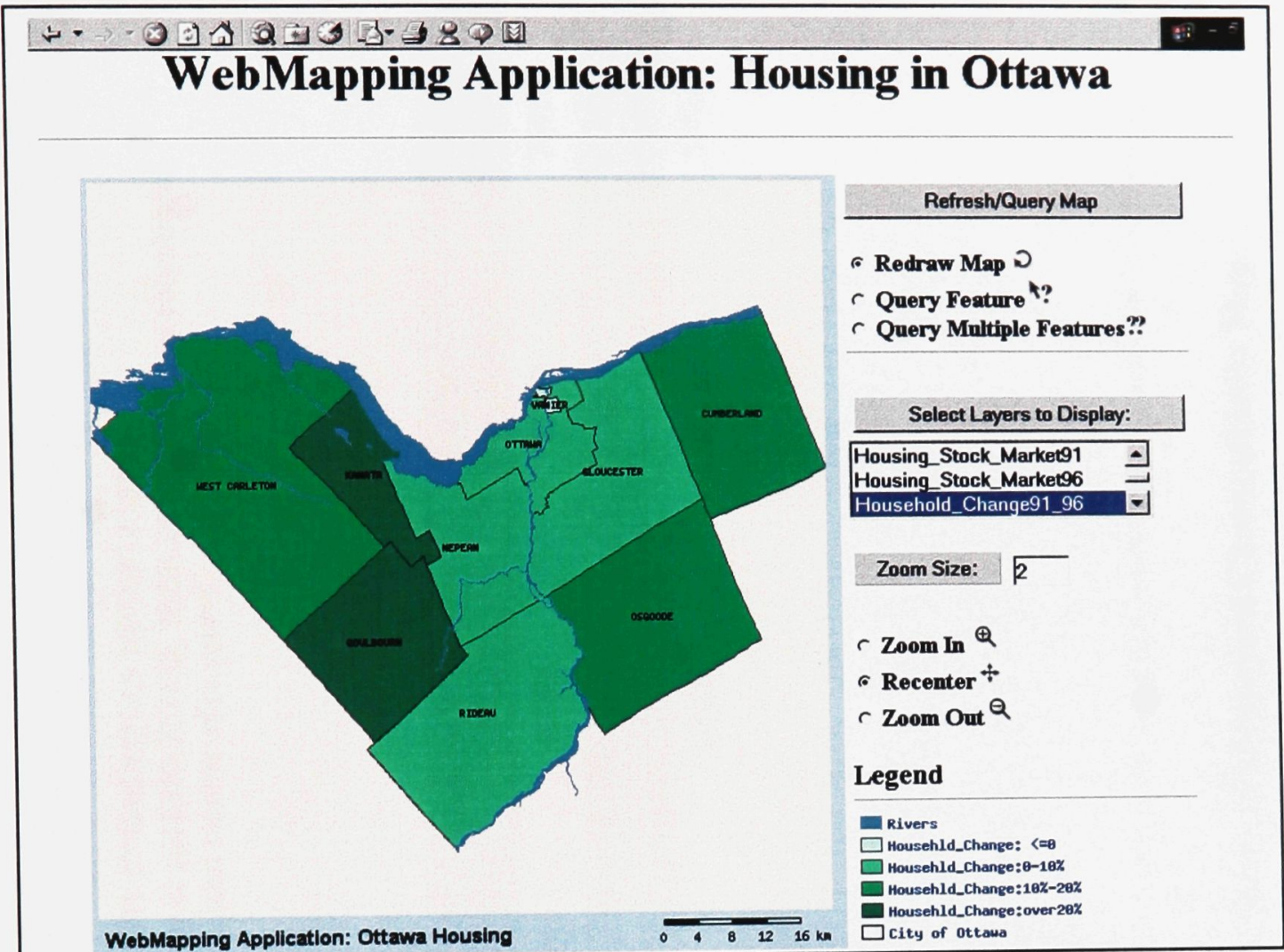

Figure 4.4.9 the Number of Household Change Distribution 1991-1996 


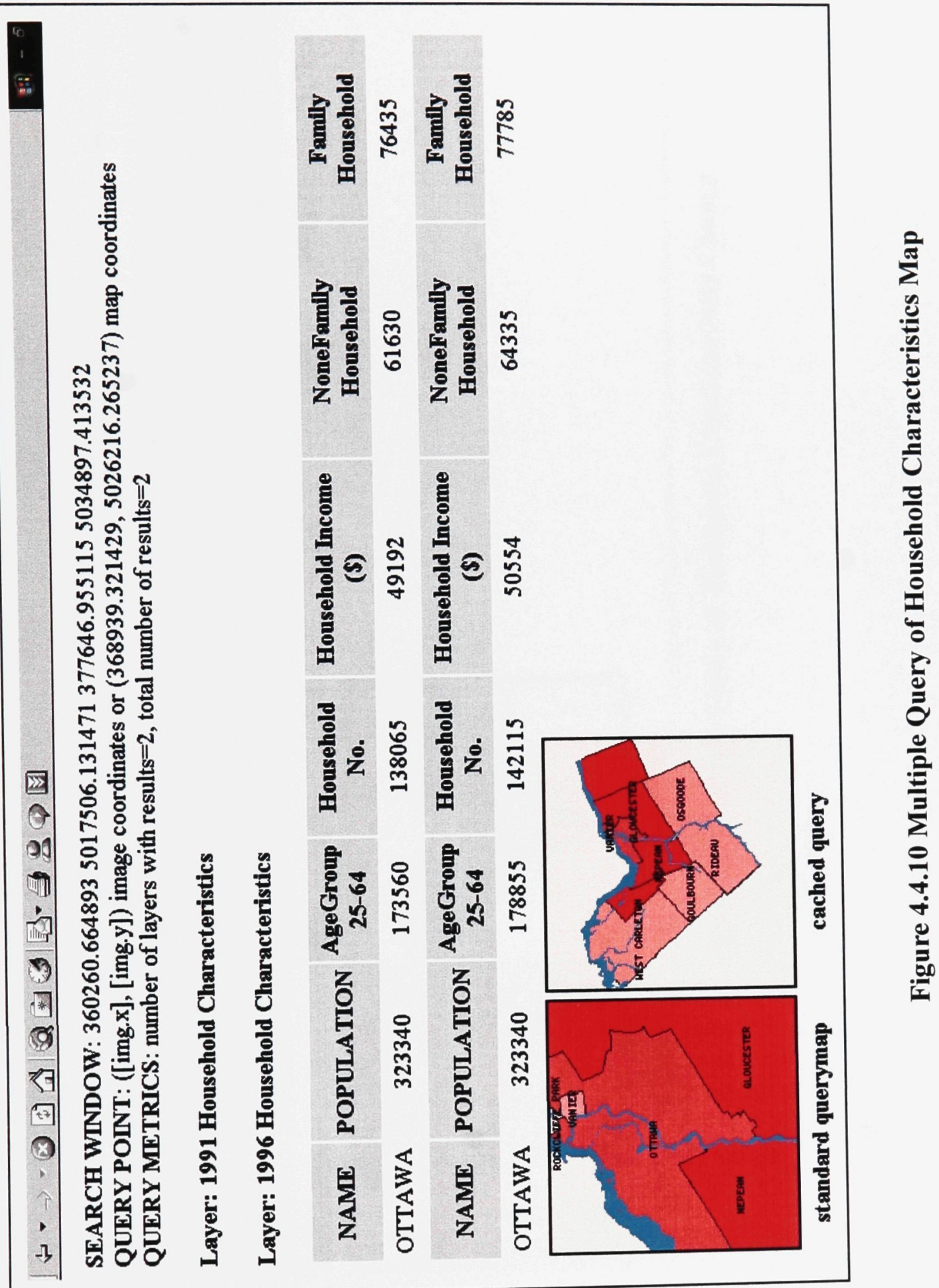


t

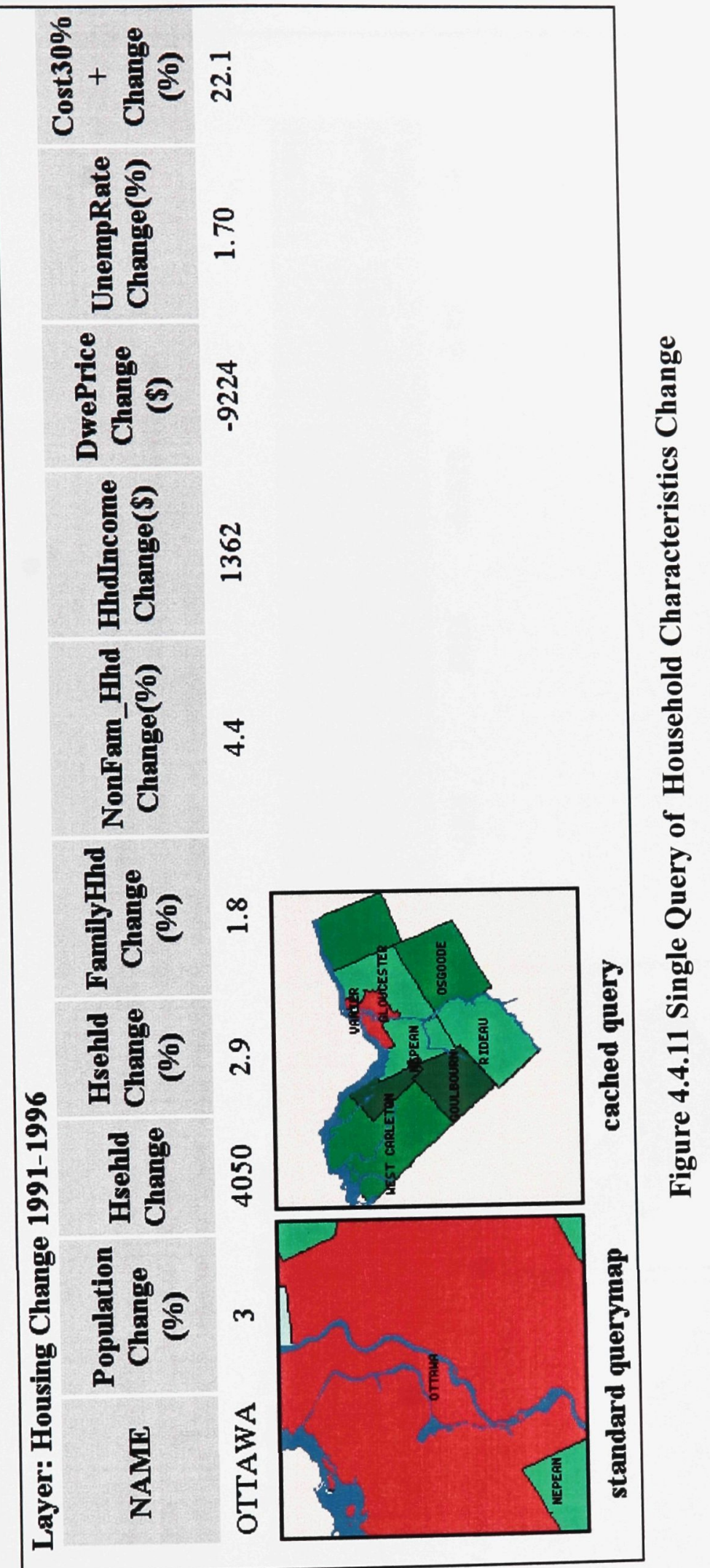




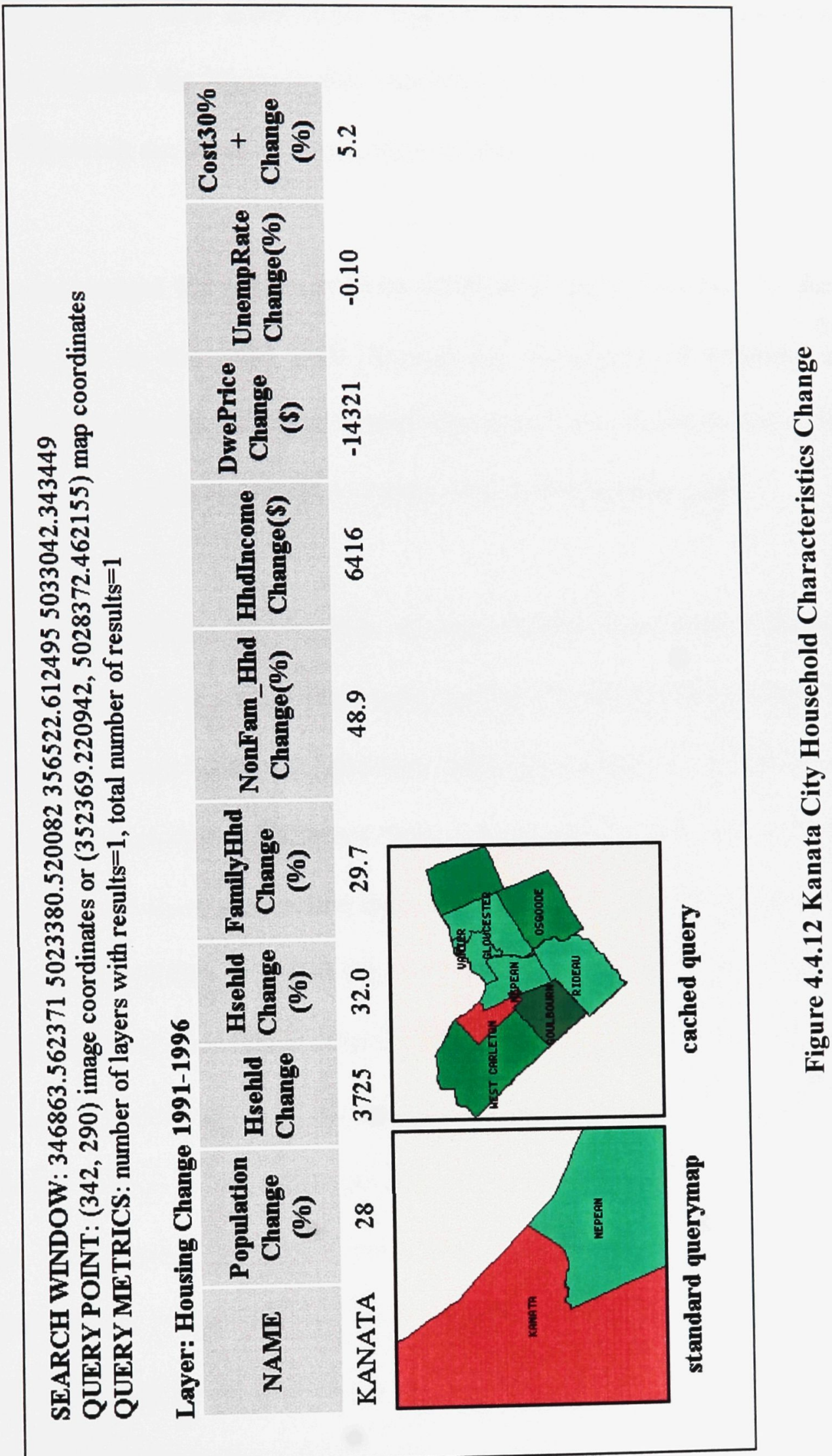


The intention of this case study is to improve the spatial data access to housing statistics and broaden the Webmapping community. Besides the outcomes described above, the following are additional important results of this study:

This study encourages the access and use of housing spatio-statistics by the public with limited GIS or computer skills through the integration of mapping and the Internet tools. The MapServer based spatial data distribution model is also extendable in other Web based spatial application realms, which have similar goals.

There are both problems and benefits existing in this open source Webmapping solution. Often, one of the most frustrating aspects of making data accessible is the myriad of formats and locations (directory path) where data is stored. In order to make the data accessible to different Web servers and browsers, the OOHA has managed to use the most compatible data format and system development tools. Its spatial data in the OOHA has used the ArcView ".shp" format which is the default spatial data format of MapServer. The OOHA interface template has been developed by HTML, i.e. the interface template files - "Ottawa.htm" and "Ottawa_init.htm" are ".htm" format which is the most popular Webpage design format and internally supported by Internet Explorer and Netscape Navigator. The map display administration tool -"Ottawa.map" file, which controls the functionalities of the OOHA, is developed by mapscript - a native data format defined by the MapServer platform. However, data compatibility problems still exist in this case although many data format issues have been carefully considered. One problem is the data display via 
Netscape Navigator. OOHA cannot be properly viewed in the Netscape Navigator 107 browser. Moreover, there are also problems evident when OOHA is browsed through old versions of Internet Explore. Thus, a further study on data interoperability is necessary to achieve better Webmapping implementation.

One of the benefits of this open source development solution is that there are no licensing fees which means the organizations with distributed data storage can focus their limited funds on publishing data. Potentially, this technology could be adapted to other applications, especially for the purpose of allowing small organizations or academics with limited budgets to publish their datasets for the broader viewer -- an economic opportunity for spatial information promotion. Since the housing issue is a significant concern for all levels of government and individuals, the benefits of this open source Webmapping approach are ideal for this case study in this context.

\subsection{Conclusion}

The study concludes that housing requirements and housing conditions within the city of Ottawa are largely determined by socio-economic and demographic factors, as well as different household characteristics. Such impact is geographically distributed and demonstrates spatial and temporal variation.

By putting housing spatial data online, access to the existing resources and housing information has been extended. The user is allowed to perform view, query and other basic GIS manipulations via the Internet without using any specialized software and from any location with the Internet access. The housing policy makers or any other 
individuals who are interested in housing information can query the database to access the locational and attribute data of housing features easily and efficiently. 


\section{CHAPTER 5. CONCLUSION AND OUTLOOK}

This chapter will summarize and conclude the research. It will address the opportunities and challenges of integrating Internet techniques with GIS technologies. Moreover, the outlook and future prospects of this particular webmapping technology and application will be discussed.

\subsection{Overview}

This research attempts to provide a vision of new opportunities and challenges offered by the modern Internet and communication technologies for the mapping and GIS industry. By integrating with the IT technology, the GIS industry is becoming more creative in managing spatial information which is very much a growth area in recent years.

With this idea in mind, an Online Ottawa Housing Atlas prototype was constructed based on an open domain CGI MapServer Engine developed at the University of Minnesota. This prototype developed a process of distributing geographic data, socio-economic statistics information, text, graphics, and images with basic dynamic and interactive geospatial manipulation functions and delivered a product distributed via Internet. The fundamental Client/Server approach of establishing web based geo-spatial network architecture was used. Several approaches have been employed in the past to study statistical information including housing needs and economic related issues in society, but few studies take the online visualization approach used in this study. The CMHC is 
currently developing a project for an online housing spatial statistics service (named "MAGIS"). Many efforts have been made to implement the process of putting national housing information online to support housing market analysis and housing policy making.

This study has explored the valuable roles that web mapping is playing in society through the application of the Online Ottawa Housing Atlas (OOHA). This application has demonstrated the importance of putting maps online in satisfying new demands for access to spatial information by both housing policy decision-makers and the general public in the expanding information society.

The OOHA application has allowed the distribution and demonstration of the spatial and temporal patterns of housing related demographic, economic and market factors, which are associated with the housing conditions and housing needs in the Ottawa area. This has been done by creating intuitive visualization device- maps through the Internet. As maps extend vision to areas much larger than are seen directly (MacEachren, 1992), so the use of OOHA has enabled the housing spatio-statistical data to be flexibly and interactively used by society in a more creative way. It is foreseeable that, by moving maps online, more and more spatial information applications will be extended into the community and will create enormous unexpected benefits for the public. 


\subsection{Opportunities and Challenges}

\section{OPPORTUNITIES}

Dynamic and interactive features extend the potential of GIS and mapping technologies. The Webmapping system, as the new communication form for map and map reader, is becoming the bridge between communities and a large volume of geospatial information data. As we are in an information era, there may well be a requirement to translate data to new media in order to preserve its utility in the future (Perkins, 1994). Webmapping, as one of the forms of cybercartography, transforms all kinds of socio-economic and environmental data into interactive representations that allow the user to explore spatial patterns and relationships in new ways (Taylor, 1997). With the successful integration of Internet and GIS technology, Webmapping allows people to manage and access the vast quantities of information with speed and flexibility through the Internet.

\section{CHALLENGES}

Implementing Webmapping applications can present a number of challenges. Several critical challenges currently faced by Webmapping application developers are discussed below.

\section{INTERFACE DESIGN}

One of the most important challenges is the interface design, i.e. how to design a proper interface to satisfy the different viewers. 
Considering the diverse compositions of user groups, with or without GIS and mapping knowledge or computer background, designers need to balance the different levels of requirements for interface design. The requirements from the professional GIS users, public audiences and the government management level users for browsing online spatial information are very different.

It is important to define the major user group of a specific application and understand their specific requirements of the online geospatial service. If the major user group is defined as the public audience, the interface should be as simple and straightforward as possible, because public users have different background in terms of GIS and map knowledge. The interface that consists of more GIS functions would inhibit them from exploring the maps. For example, the OOHA application is mainly designed for the general public and government decision makers. This group of users has only limited GIS and map knowledge. Therefore, the generic map interface with the basic map view and query tools has been developed to represent the housing statistics. (Figure 4.3.3.1.1 Online Ottawa Housing Application Interface)

For the interface design requirements, a general user survey can be easily established through Internet by a form questionaire. This research is beyond the study scope of this thesis due to time restrictions. However, a further study to the Webmapping interface design is necessary for implementing a successful Webmapping application. 


\section{INTEROPERABILITY}

Interoperability of the system is another critical point that needs to be taken into consideration. On the Internet, a number of heterogeneous applications and computers are usually connected. Successful communication between each is required. However, finding usable solutions for interoperability is one of the demanding challenges in Webmapping application design. Open GIS Consortium (OGC) has set up significant initiatives in respect to interoperability issues. Although the important standards, including web environment, data format, computing architecture have been defined by OGC, it still takes time to adapt all existing software, hardware and data to be OGCcompliant. Additional efforts need to be continuously made to address this important issue.

\section{PERFORMANCE AND FUNCTIONALITY}

Most of the Webmapping applications take some time to generate a map, especially when the system is running analyses such as classification or a complex multiple queries. This has large impacts on the use of the Webmapping system. The possible reasons of this slow performance can be the bandwidth of the Internet, strategy design and the capability of the hardware and software, such as memory, disk space and computation processor resources. In particular, this brings limitations to the function design of a Webmapping system. The more complicated the Webmapping functionality is, the longer waiting time for the user. This is usually not acceptable to most public audiences. With the development of IT technology, it is expected that most of these problems will eventually 
be lessened as general computing and network technology improves.

\section{STRATEGY DESIGN}

Strategy design limited by current technology is another challenge worth mentioning. The listed fundamental strategies within Chapter 2 are suitable for today. However, technology is rapidly changing especially in the computer and communication industries. It is likely that new methods will be available in the future that can run the system faster and more efficiently, and allow the developer to include features not possible today. It is also possible that there are some approaches in which the solutions built today are not ideal for tomorrow.

\subsection{Outlook}

The results of this thesis suggest that GIS and mapping technology, visualization and multimedia representation, spatial socio-economic data sources, and the Internet tools will blend into one seamless computing environment. This integration will have a significant influence on the way people think about and communicate spatial and temporal issues.

With the successful merging of mapping technology and the Internet, we can finally integrate our knowledge of geomatics into our daily life. Geospatial information has become fast, flexible, and easily accessible to the public. The age of societal GIS is upon us (Harder, 1998). It indicates that geospatial information becomes part of the framework of our conscious lives, touching every aspect of our daily existence in unprecedented ways. 


\subsection{Future Research}

It was the primary goal that this research yield results leading to the examination of the proposed fundamental approach of establishing web based geo-spatial networks and advance the roles of Webmapping to serve society. As technology does not stand still, ongoing further studies are necessary in order to fully incorporate new emerging technology into the Geomatics industry and extend geospatial information into society. 


\section{Appendix: Glossary of Internet Terminology}

\section{BANDWIDTH}

This term refers to the rate at which data can be sent over a connection between two computers on a network. As with any flow, bandwidth is measured as the volume of data passed in a given amount of time, expressed either in a kilobits per second (Kbps or "kilo-baud"), megabits per second (Mbps) or megabytes per second (Mbps). A high bandwidth line such as T3 (45 Mbps), can send larger files in less time than a low bandwidth line, such as a 28.8 -Kbps modem.

\section{BROWSER PLUG-IN}

A small program that can be attached to a Web browser to enhance its capabilities. The most common plug-ins are used to view specialized types of data such as video, animations, and complex graphic ( e.g., maps). Each plug-in is registered with the browser for certain types of information. When items of those types information are requested by the user, the plug-in is activated to handle them.

\section{CGI}

Common Gateway Interface. An interface -creation scripting program that allows on the fly creation of WWW pages based on information from buttons, check boxes, text input, and so on. 


\section{CLIENT/SERVER MODEL}

A common architecture for distributed computing applications, such as the Internet or a large GIS installation. Information and applications are stored on one or more servers, which can be accessed by any number of clients on computers connected to a network.

\section{DOMAIN NAME SERVICE (DNS)}

A standard program on the Internet that allows you to use an alphanumeric name ( called a "host name") for a computer in lieu of the numeric IP address. DNS names each machine in terms of its top-level domain (e.g., ".com", ".edu", and ".ca"), organization domain that mimics the name of the organization (e.g., "carleton", "umn"), optional subnetwork designator representing a department or office (e.g., "geography”), and machine name (e.g., www).

\section{MIDDLEWARE}

The middleware technology refers to the the technologies like CGI Script, DCOM, CORBA that break the information into small packets and check that the packets were received by the client computer on the other side of the transaction.

\section{HTML/DHTML}

HyperText Markup Language/ Dynamic Hypertext Markup Language. The programming language in which World Wide Web documents are written. 


\section{HTTP}

HyperText Transfer Protocol. The common protocol used to transmit documents on the World Wide Web.

\section{IP ADDRESS}

In order to facilitate communication between computers on the Internet, each computer is assigned a unique address. This consists of a four-byte number, usually written as each byte separately with periods in between, such as 200.118.117.110. Using the Domain Naming Service, an alphanumeric name can be assigned to each address, such as "chat.carleton.ca", which is easier to remember.

\section{INTERNET}

A decentralized computer network linking tens of thousands of smaller networks and accessed by millions of users worldwide. Users connected to the Internet can send and received emails, download files, view multimedia content on the world Wide Web. There is no single organization owns or controls the entire network, although it is generally controlled by a series of accepted standards. The World Wide Web is only one form of communication that takes place over the Internet. 


\section{INTRANET}

A system similar to the Internet but used to communicate only within an organization. It uses standard Internet protocols - such as HTTP, SMPT and TCP/IP, and is based on the same distributed Client/Server idea. However, everything is located on the local area network, and is usually barred from the rest of the Internet by a firewall.

\section{JAVASCRIPT}

A derivative of Java used to add simple functionality to HTML documents and HTTP servers.

\section{PROTOCOL}

A language by which two computers communicate. Common Internet protocols include HTTP and TCP/IP. A protocol normally consists of a standard format for issuing requests and commands, as well as delivering prescribed forms of information.

During a communication, many protocols may be used simultaneously on different levels. For example, when you retrieve a page on the Web, the HTTP protocol is used to format the file so that the browser can understand what to do with it.However, TCP is used to actually manage the file transfer, and at an even lower level, IP controls the physical transmission of the data from one computer to another. 


\section{TCP/IP}

Transmission Control Protocol/Internet Protocol. The group of protocols that defines the Internet. Origionally created for the UNIX operation system, TCP/IP software is now usable for every major computer operation system. In order to be on the Internet, a computer must have TCP/IP software.

\section{URL}

Uniform Resource Locator. The address for a site on the World Wide Web, for example: http://www.carleton.ca

\section{VRML}

Virtual Reality Modeling Language. A standard data mode used to describe three dimensional environments.

\section{WORLD WIDE WEB (WWW)}

One form of the communication on the Internet, alongside system such as FTP, email, and chat. Although based on the foundation of the HTML data format and HTTP protocol, it has grown to include many other types of information, such as graphics and JavaScript applications. 


\section{References}

Adrienko, G. and Adrienko, N.V. (1999), "Interactive Maps for Visual Data

Exploration”, Int. J. Geographical Information Science, 1999, Vol.13, No.4, 355-374.

Alexander, J.E. and Tate, Marsha (1999), Web Wisdom: How to Evaluate and Create Information Quality on the Web, Lawrence Erlbaum Associates, New Jersey.

Bima, William J. (2001), “Data Management Solution”, GEOWorld, July, 2001, P38-41.

Cammack, R.G.(1999), “ New Map Design Challenges: Interactive Map Products for the World Wide Web", Chapter 6 in Cartwright, William, Peterson,M.P. and Gartner, G. (Eds.) Multimedia Cartography, Springer, New York.

Canada Mortgage and Housing Corporation, 1995, Changing Values Changing Communities: A Guide to the Development of Healthy, Sustainable Communities, June, 1996.

Canada Mortgage and Housing Corporation, 1984, Housing in Canada, A Statistical Profile, Nov. 1984.

Canada Mortgage and Housing Corporation, 1991, Technical Guide to Understanding 1991 Core Housing Need Data Bases and Estimates.

Canada Mortgage and Housing Corporation, 1999, Summary of the Corporate Plan, CMHC 1999-2003.

Canada Mortgage and Housing Corporation, 2000, Canadian Housing Statistics 2000.

Canada Mortgage and Housing Corporation, 1999, "Economic Impacts of Residential Construction", in Research Highlights, Socio-economic Series, Issue69 
Canada Mortgage and Housing Corporation, (1999), Summary of the Corporate Plan, CMHC 1999-2003.

Canada Mortgage and Housing Corporation, (1994), Case Study: Alternative Development Standards for Affordable Housing in Ottawa-Carleton.

Canada Mortgage and Housing Corporation, (2001), "Special Studies on 1996 Census Data: Canadian Households in Core Housing Needs and Spending At Least Half Their Income on Shelter", in Research Highlights, Socio-economic Series, Issue55-7, May 2001.

Canada Mortgage and Housing Corporation, "Demographic Changes and Real Housing Prices in Canada", in Research Highlights, Socio-economic Series, Issue62

Canada Mortgage and Housing Corporation, 1999, "Housing Need in Canada's Metropolitan Areas, 1991”, Research Highlights, Socio-economic Series, Issue 18

Canada Mortgage and Housing Corporation,1973, Demographic and Economic Aspects of Housing Canada's Elderly.

Canada Mortgage and Housing Corporation, Ottawa Local Housing Market Report, 1991, 1993, 1995 \& 1996.

Canada Mortgage and Housing Corporation, Ottawa Housing Forcast, 1991, 1995\& 1996.

Carriere, J.(1999), “Atlas du Quebec et de ses Regions”, Chapter 11 in Cartwright, W., Peterson,M.P. and Gartner, G. (Eds.) Multimedia Cartography, Springer, New York. 
Corbley, Kevin (2000), "Government that Works: Massachusetts Town Provides

Property Data Online”, GEOWorld, December 2000, P40-42, http://www.geoplace.com

Cartwright, W.(1995), "Multimedia and Mapping: Using Multimedia Design and Authoring Techniques to Assemble Interactive Map and Atlas Products", in $17^{\text {th }}$ ICA, Barcelona, Spain, Proceedings, P1116-1127.

Dransch, D. (1998), The Use of Different Media in Visualisation Spatial Data, Computers $\&$ Geosciences 26 (2000) 5-9.

Forsythe, C., Grose,E. and Ratner J. (1998), Human Factors and Web Development, Lawrence Erlbaum Associates, New Jersey.

Fuhrmann, S. (1998), " Design a Visualization System for Hydrological Data”, Computers \& GeoSciences 26 (2000), P11-19

Goodchild, M.F.(1999), “Cartographic Futures in a Digital Earth”, in Keller,P. (ed.) (1999), Touch the Past, Visualize the Future, Ottawa ICA 1999 Conference Proceedings, Vol.1,P5-13.

Goodchild, Michael (2000), "Postscript: New Directions for GIS Research", In David Davis (Ed) GIS for Everyone, ESRI Press 2000, P588 -595.

Green, D.R. (1997), “Cartography and the Internet”, The Cartographic Journal. 34, 1, P23-27.

Harder, C.(1998), Serving Maps on the Internet, ESRI, California.

Hartman, Chester W.(1975), Housing and Social Policy, Prentice-Hall, Inc., Englewood Cliffs, N.J. 
Hetch, L.(2001), "OGC Pilot Takes Web Mapping to a New Level”, GeoWorld, June 2001, P32.

Huang,B., Jiang, B., and Li, H. (1999), “ An Integration of GIS, Virtual Reality and the Internet for Visualisation, Analysis and Exploration of Spatial Data", International Journal Geographical Information Science, 2001, Vol.15, No.5, 439-456, http://www.tandf.co.uk/journals

Kahkonen, J., Lehto, L., Kilpelainen, T.and Sarjakoski, T.(1999), "Interactive Visualisation of Geographical Objects on the Internet", International Journal Geographical Information Science, 1999, Vol.13, No.4, 429-438, http://www.fgi.fi//osastot/karto/projektit/netgis/vi_98/ngsh.html

Kraak, M.J. and Brown,A.(2001), Web Cartography, Taylor and Fransis, New York

Limp, W.F. (2001), “User Needs Drive Web Mapping Product Selection”, GEOWorld, Februry 2001, P8-14.

MacEachren, A.M., Buttenfield, C., Dibiase,D.W. and Monmonier, M.(1992), "Visualisation" Chapter 6 in Alber,R.F. Marcus,M.G. and Olson,J. Geography's Inner Worlds, Rutgers University Press.

Meyerson, M., Terrett, B. and Wheaton, W.(1962), Housing, People and Cities, McGrawHill Book Company, Inc, New York.

Planning Department, City of Ottawa, 1992, 1991 Annual Housing Review

Planning Department, City of Ottawa, Regional Housing Statement Update, 1985\& 1991

Plewe, B. (1997), GIS Online: Information Retrieval, Mapping, and Internet, OnWord Press, 1997. 
Prescott, J.R. (1974), Economic Aspects of Public Housing, Sage Publications, Beverly Hill, London.

Raper, J. (2000), Multidimensional Geographic Information Sciences, Taylor \& Francis, London and New York.

Taylor, D.R.F. (1997), "Maps and Mapping in the Information Era", Keynote address to the $18^{\text {th }}$ ICA Conference Stockholm in Ottoson, (ed.) Proceedings. Vol.1, P1-10

Zeng, T.Q.(1999), “Optimal Spatial Decision Making Using GIS: A Prototype of a Real Estate Geographical Information System”, International Journal Geographical Information Science, 2001, Vol.15, No.4, 307-321,

Wise, S., Haining, R., and Ma, J.Sh. (1999), "Providing Spatial Statistical data Analysis Functionality for the GIS user: the SAGE Project", International Journal Geographical Information Science, 2001, Vol.15, No.3, 239-254, http://www.tandf.co.uk/journals 


\section{URLs:}

Autodesk: http://autodesk.com

City of Ottawa Atlas: http: http://atlas.city.ottawa.on.ca/mapping/atlas/atlas.htm

Cabarrus County: http://co. cabarrus.nc.us/atlas.htm

ESRI: http://www.esri.com

GeoWorld: http://www.geoworld .com

Intergraph: http://www.intergraph.com

MapInfo: http://mapinfo.run

MapServer: http://mapserver.gis.umn.edu

MapQuest: $\underline{\text { http://www.mapquest.com }}$

MapBlast: http://www.mapblast.com

National Atlas of Canada: http://atlas.gc.ca/

National Atlas of the United States: http://nationalatlas.gov/

National Geospatial Data Clearinghouse of the united States:

http://www.fgdc.gov/clearinghouse

Open GIS Consortium: http://www.opengis.org/

WWW Consortium: http://www.w3.org/ 